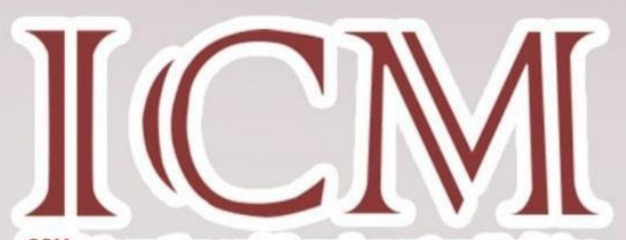

овм Integrative and Complementary Medicine

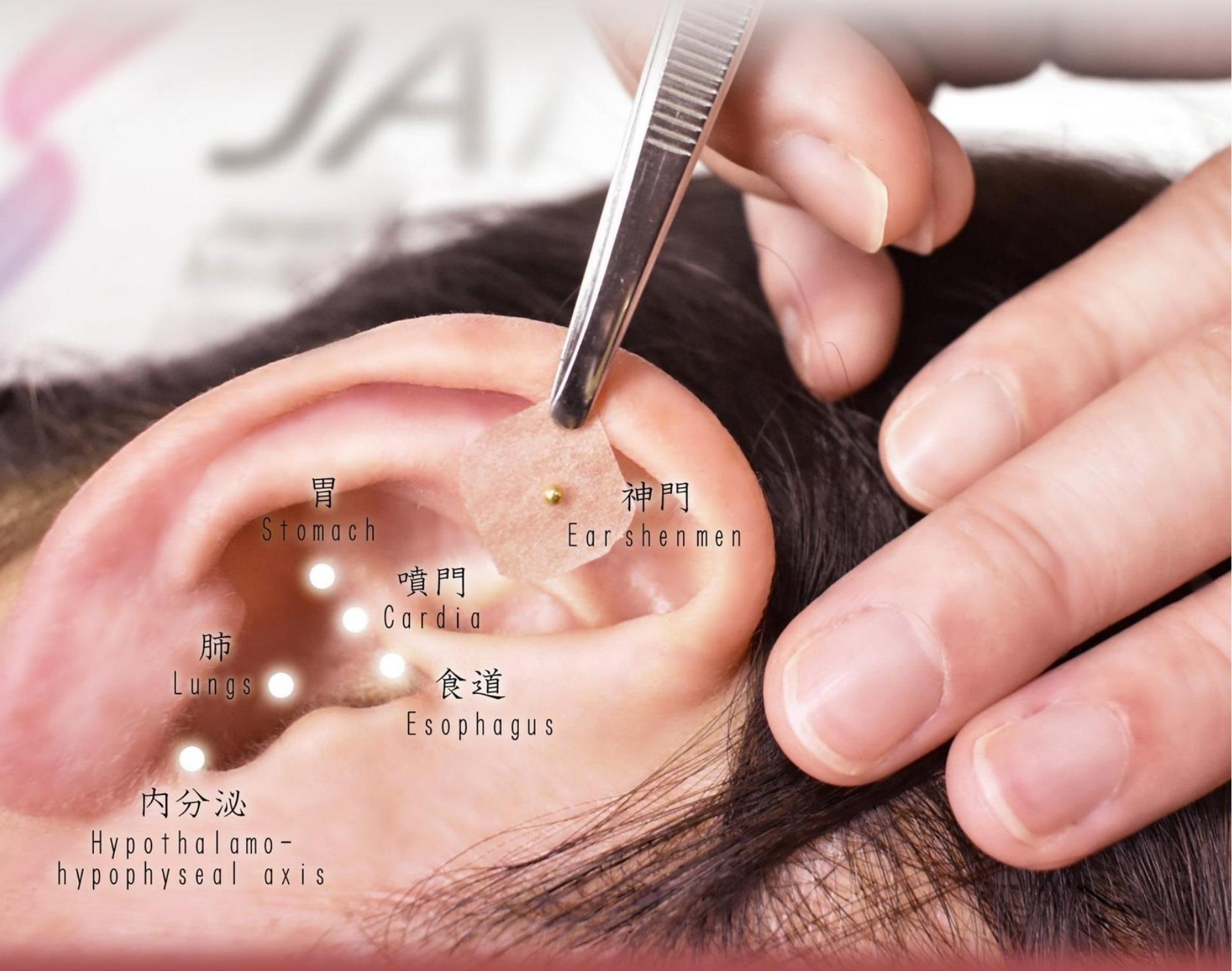

\title{
Auricular Acupuncture with Beads Supports Sustained Weight Loss
}

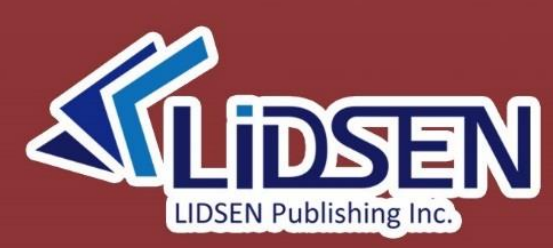


$O B M$ Integrative and

Complementary Medicine

\section{OBM Integrative and \\ Complementary Medicine \\ 2020 Volume 5, Issue 4}

Editor-in-Chief

Professor Gerhard Litscher

Printed Edition Published in 
Editorial Office

$O B M$ Integrative and Complementary Medicine Editorial Office, 73 Hongkong Middle Road, Qingdao, China

Tel./Fax: +86-532-8979-9572

LIDSEN Publishing Inc.

2000 Auburn Drive, One Chagrin

Highlands, Suite 200, Beachwood, $\mathrm{OH} 44122$, USA

Tel.: +1-216-370-7293

Fax: +1-216-378-7505

http://www.lidsen.com/

This is a reprint of articles from the Issue 4 published online in the open access journal OBM Integrative and Complementary Medicine (ISSN 2573-4393) from October 1, 2020 to December 31, 2020.

Available at: http://www.lidsen.com/journals/icm/icm-05-04

For citation purposes, cite each article independently as indicated on the article page online and as indicated below:

LastName, A.A, LastName, B.B, LastName, C.C. Article Title. Journal Name Year; Volume(Issue):Article Number; doi.

(C) 2020 by the author. This is an open access article distributed under the conditions of the Creative Commons by Attribution License, which permits unrestricted use, distribution, and reproduction in any medium or format, provided the original work is correctly cited. 


\section{Contents}

\section{Deborah R. Simkin, L. Eugene Arnold}

The Roles of Inflammation, Oxidative Stress and the Gut-Brain Axis in Treatment Refractory Depression in Youth: Complementary and Integrative Medicine Interventions

Reprinted from: OBM Integrative and Complementary Medicine 2020;5(4):24; doi:10.21926/obm.icm.2004040

Emily Reeves, Brigitte Liebig, Eveline Degen Jermann

Comparing the Perspectives of Professionals and Relatives Toward the Quality of Palliative Care: A Qualitative Study

Reprinted from: OBM Integrative and Complementary Medicine 2020;5(4):20; doi:10.21926/obm.icm.2004041

Vasilios M. Polymeropoulos

A Potential Role of Coenzyme Q10 Deficiency in Severe SARS-CoV2 Infection

Reprinted from: OBM Integrative and Complementary Medicine 2020;5(4):8; doi:10.21926/obm.icm.2004042

Gerhard Litscher

Dynamic Pulse Reaction - Update 2020

Reprinted from: OBM Integrative and Complementary Medicine 2020;5(4):5; doi:10.21926/obm.icm.2004043

Wangkuk Son, Jason Lin, Melissa Puppa

Nutritional Interventions in Cancer Cachexia Prevention and Treatment

Reprinted from: OBM Integrative and Complementary Medicine 2020;5(4):24; doi:10.21926/obm.icm.2004044

Takahiro Fujimoto, Hidetake Kobayashi, Takeshi Hataoka, Kazuo Taniguchi, Keisuke Miura Auricular Acupuncture with Beads Supports Sustained Weight Loss

Reprinted from: OBM Integrative and Complementary Medicine 2020;5(4):18; doi:10.21926/obm.icm.2004045 
Review

\title{
The Roles of Inflammation, Oxidative Stress and the Gut-Brain Axis in Treatment Refractory Depression in Youth: Complementary and Integrative Medicine Interventions
}

\author{
Deborah R. Simkin ${ }^{1,}{ }^{*}$, L. Eugene Arnold ${ }^{2}$
}

1. Department of Psychiatry, Emory School of Medicine, 4641 Gulfstarr Dr. Suite 106, Destin, Florida 32541, USA; E-Mail: Deb62288@aol.com

2. Professor Emeritus of Psychiatry \& Behavioral Health, College of Medicine, Ohio State University, USA; E-Mail: L.Arnold@osumc.edu

* Correspondence: Deborah R. Simkin; E-Mail: Deb62288@aol.com

Academic Editor: Bettina Bernstein

Special Issue: Integrative Therapies to Address Mood and Anxiety Symptoms in Youth and Young People

OBM Integrative and Complementary Medicine Received: April 06, 2020

2020 , volume 5 , issue 4

Accepted: September 22, 2020

doi:10.21926/obm.icm.2004040

Published: October 19, 2020

\begin{abstract}
Teen depression and suicide rates have risen despite conventional treatments. This article reviews adjunctive interventions that may improve outcomes. A search of the National Library of Medicine database used tailored searches with combinations of specific terms. Modern lifestyle is associated with increased inflammation and pro-inflammatory cytokines leading to, for instance, hyperactivation of the hypothalamic-pituitary-adrenal axis, which promotes depression. Inflammation also increases oxidative stress, leading to mitochondrial dysfunction, also associated with depression. Diets with less probiotic-containing fermented foods change the microbiome and decrease the bio-availability of mood-regulating $B$ vitamins crucial to neurotransmitter production. Vitamin D deficiency allows increased pro-inflammatory cytokines and disrupts mitochondrial function and monoamine production. Deficiencies/insufficiencies of magnesium, Vitamin D, and B vitamins correlate with
\end{abstract}

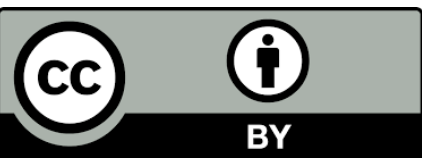


depression severity. Deficiencies of the folate and methylation cycles may lead to treatmentresistant depression. Imbalance of omega- 6 and omega-3 fatty acid intake allows more proinflammatory eicosanoids (prostaglandins, thromboxanes, leukotrienes) from omega- 6 than anti-inflammatory eicosanoids from omega-3. Refractory youth depression may be linked to abnormalities in functional biological systems, with excessive inflammation, oxidative stress, and gut-brain issues. Mediterranean diet, vitamins/minerals, omega-3 fatty acids, methyl donors, meditation, and exercise are worth considering as adjunctive treatments. More research is needed.

\section{Keywords}

Complimentary and Integrative psychiatry; depression; functional medicine; omega 3 fatty acids; adolescents; inflammation; oxidative stress; gut-brain axis; hypothalamicpituitaryadrenalaxis; diet; vitamin deficiencies

\section{Introduction}

Youth depression (ages 12-20) increased 37\% from 2004 to 2014 [1]. This coincided with a ninefold increase in suicidal ideation in adolescents [2]. Suicide rates among females has increased by $65 \%$ since 2010 [3]. Some youth are refractory to antidepressants [4, 5]. Functional Medicine (which analyzes abnormalities in biologic functional systems) and Complementary and Integrative Medicine (CIM) interventions are being studied to address reasons for and interventions for treatment-refractory depression. Treatment refractory depression here is defined as any depression for which conventional/traditional treatments have not been successful.

Inflammation disrupts multiple pathways relevant to the risk of developing depression. Inflammation in the gut during stress, for instance, increases production of pro-inflammatory cytokines which stimulates the vagal nerve. The subsequent effect is that the hypothalamicpituitary-adrenal (HPA) axis is upregulated, with adrenaline and cortisol increases. Sustained increases in cortisol and pro-inflammatory cytokines have been associated with depression [6]. Proinflammatory cytokines interfere with the indoleamine 2,3-dioxygenase (IDO) pathway, which decreases neurotransmitter production and disrupts microglia, leading to depressive symptoms [7]. Inflammation can also lead to oxidative stress which depletes glutathione needed to neutralize reactive oxygen species (ROS). The overabundance of ROS leads to mitochondrial dysfunction. Damage to the mitochondrial electron transport chain has been associated with depression and bipolar disorder [8]. Inflammation can also disrupt the folate and methylation cycles by decreasing the absorption of vitamin B12. B12 also plays a major role in neurotransmitter production (Figure 1).

Inflammation has been linked to obesity and diet. High-processed-fat and high-sugar foods cause dysbiosis or changes in the gut that cause inflammation and intestinal permeability that leads to insulin resistance and weight gain [9]. Processed foods, sugary beverages, and sedentary habits leading to weight gain are associated with a higher incidence of depression [10]. One study found that adolescents who were depressed had a $70 \%$ increased risk of being obese and obese adolescents had a $40 \%$ increased risk of being depressed [11]. 
Other modern factors that may relate to disruption of the microbiome and production of proinflammatory cytokines include pesticides [12], increased stress [6], and antibiotics [13].

Functional Medicine has begun to recognize abnormalities in biological systems that may prevent full recovery from depression. Complementary and Integrative Medicine (CIM) interventions to address these abnormalities may be used adjunctively.

Background Physiology/Immunology of Refractory Depression (Figure 1):

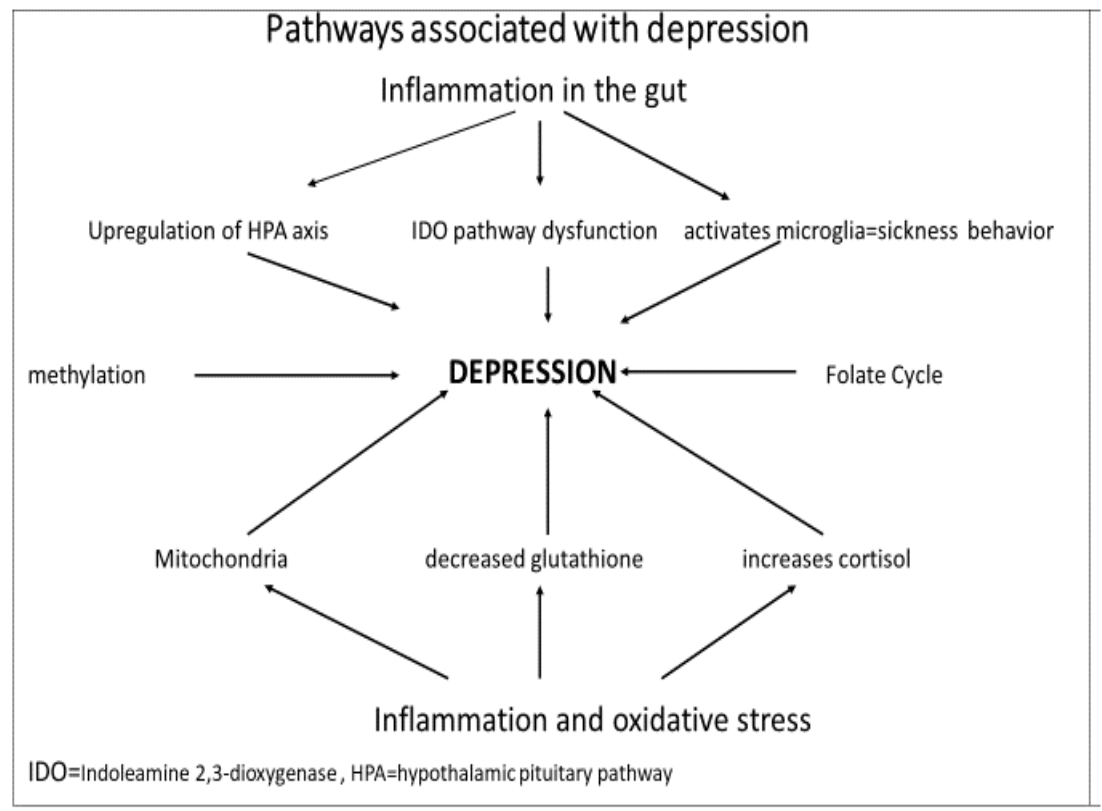

Figure 1

\subsection{Gut-Brain Axis}

A number of articles document dysbiosis-caused production of pro-inflammatory cytokines in the gut leading to psychiatric symptoms associated with depression $[6,14,15]$. In one study norepinephrine (induced by stress) caused an increase in E. coli [16] and decreases in Lactobacillus and Bifidobacterium, leading to increased lipopolysaccharides (LPS) [14]. LPS stimulate lymphocyte differentiation, leading to production of pro-inflammatory interleukin -beta (IL- $\beta$ ), interleukin-6 (IL6 ), and tumor necrosis factor alpha (TNF $\alpha$ ) [17]. TNF $\alpha$ compromises mucosal integrity by promoting disintegration of tight-junction proteins, like occludin, in the gut wall. Pro-inflammatory cytokines also decrease short chain fatty acids (SCFA), like butyrate, acetate and propionate, responsible for maintaining gut integrity $[15,18]$. Hence dysbiosis can lead to "leaky gut" $[6,7,15,18]$. Leaky gut leads to a corresponding breakdown of the blood-brain barrier, which allows inflammatory cytokines to enter the brain. Leaky gut can increase the risk for developing depressive symptoms in three ways:

First, IL- 6 and TNF $\alpha$ stimulate the afferent fibers of the vagal nerve, which in turn stimulates the hypothalamic-pituitary-adrenal (HPA) axis. Normally this would cause a rise in norepinephrine and cortisol and cortisol would signal the HPA axis to normalize. During chronic stress with dysbiosis, the normal negative feedback loop from glucocorticoid is impaired $[15,18]$. The release of CRF from the hypothalamus is further potentiated by the circulating pro-inflammatory cytokines. Hence, the HPA axis becomes hyperactive, a frequent finding in patients with depression (Figure 1). Pro- 
inflammatory cytokines have been associated with anhedonia in adolescents [19]. In a study where adolescents collected saliva at waking and 30-minutes past waking for 3 days, greater cortisol awakening response (CAR) predicted greater depressive symptoms, and interacted with acute interpersonal stress in predicting depressive symptoms [20].

Second, "Sickness Behavior" (depressive symptoms) is associated with activation of microglia. Microglia can be activated by cytokines produced by macrophages and monocytes that cross the blood- brain barrier, by increased levels of glutamate following acute stress exposure [21], and by increased gut LPS stimulating the afferent vagal nerve [22]. In turn, excess microglia stimulation can lead to excess cytokines (IL-beta and TNF alpha) which impair synaptic plasticity. The result is neuronal dysfunction with neuro-vegetative symptoms similar to depression (Figure 1) [23].

Third, increased proinflammatory cytokines activate Indoleamine 2,3-dioxygenase (IDO). IDO and its subsequent enzyme kynurenine monooxygenase divert tryptophan from serotonin production towards kynurenine, 3-hydroxykynurenine and subsequent quinolinic acid, a strong agonist of the glutamatergic $\mathrm{N}$-methyl-d-aspartate receptor, which has been linked to depression. In addition, all three of these aforementioned substances are associated with oxidative damage, inflammation, mitochondrial dysfunction, neurotoxicity, and lowered neuroplasticity [24]. Activation of IDO, measured by kynurenine-to-tryptophan ratio, correlates with severity of depressive symptoms [25] (Figures 1 and Figure 2).

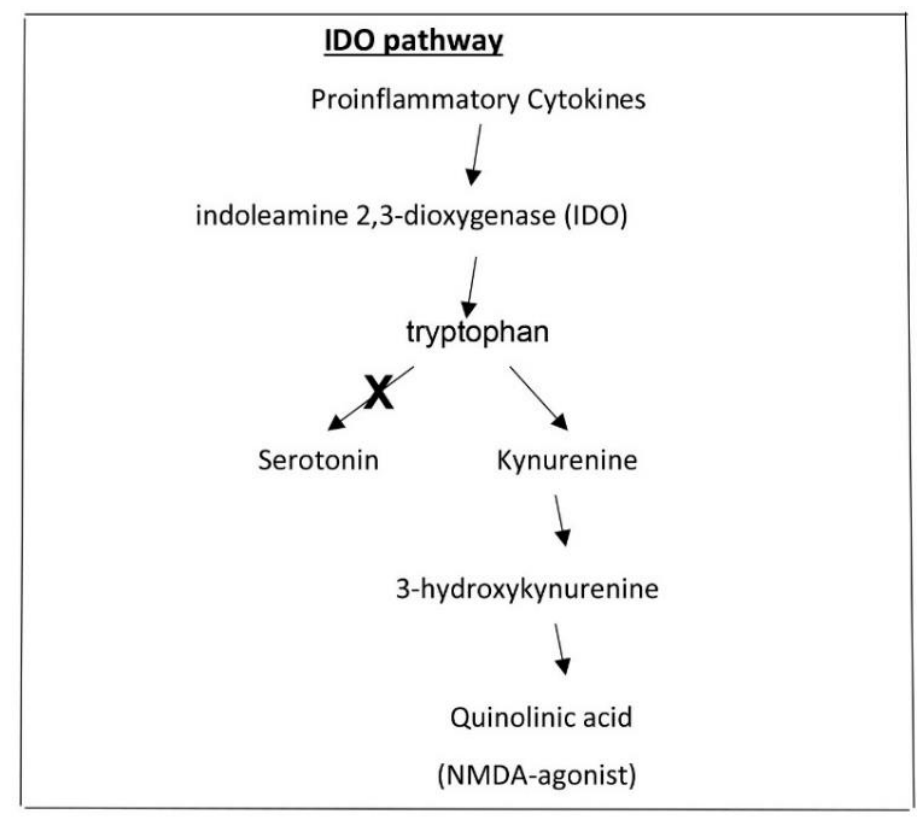

Figure 2

\subsection{Inflammation, Oxidative Stress and Glutathione}

Inflammation or production of inflammatory cytokines can lead to oxidative stress. Oxidative stress involves the formation of Reactive Oxygen Species (ROS) or free radicals. Mitochondria can normally neutralize these free radicals [26]. However, when prolonged inflammation occurs, as in active mania, the antioxidant glutathione, is decreased resulting in tissue damage [27]. Reduced glutathione $(\mathrm{GSH})$ is sacrificed by glutathione peroxidase to the oxidized disulfide form (GSSH) to neutralize ROS (See Figures 1 and Figure 3.) Mitochondrial dysfunction (Figure 1) results when the 
mitochondria are overwhelmed with the production of ROS and this results in damage to the mitochondrial electron transport chain, which has been associated with depression and bipolar disorder [28].

\section{Glutathione Conjugation}

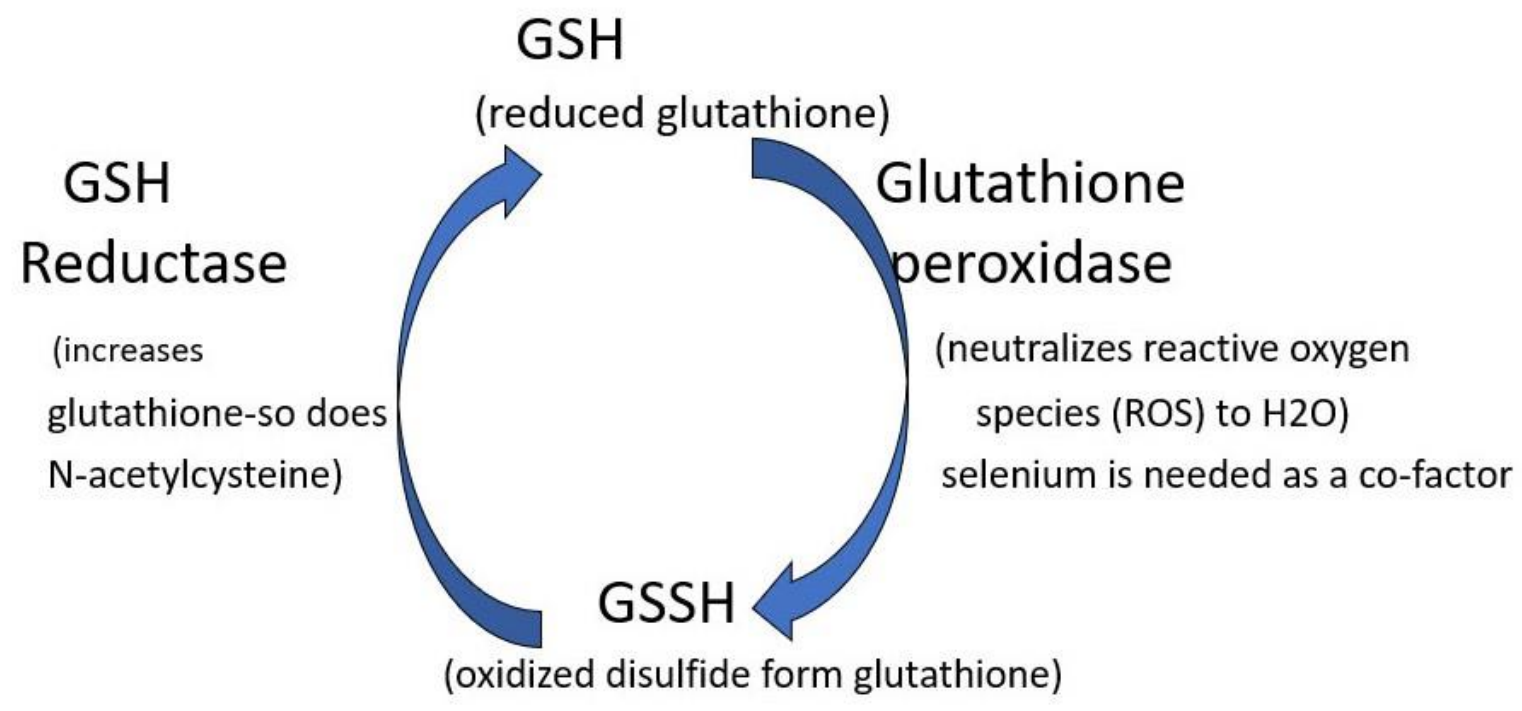

- Anti-oxidant molecules include CoQ10, alpha lipoic acid and primarily glutathione (increased with NAC) (Salim, 2015, Vavakova, 2015)

Figure 3

\subsection{B12 In the Folate and Methylation Cycles and the Effect of MTHFR Variations; Role of Gut Inflammation}

Inflammation in the gut can prevent the absorption of vitamin B12 and folate $[29,30]$. This can lead to a corresponding decrease in S-adenosylmethionine (SAMe) and increased homocysteine (Figure 3). High homocysteine levels have been associated with depression [31]. If the methylene tetrahydrofolate reductase (MTHFR) gene C677T encodes a polymorphism variant of $\mathrm{C} / \mathrm{T}, \mathrm{T} / \mathrm{T}$ or $\mathrm{A} / \mathrm{C}$, a lower proportion of dietary folate is converted to L-methylfolate in the folate cycle and less homocysteine is utilized to produce SAMe with a corresponding decrease in neurotransmitter production, possibly leading to depression (Figure 4). In children with a history of childhood trauma, the MTHFR T/T genotype carriers developed a more severe form of depression [32]. 


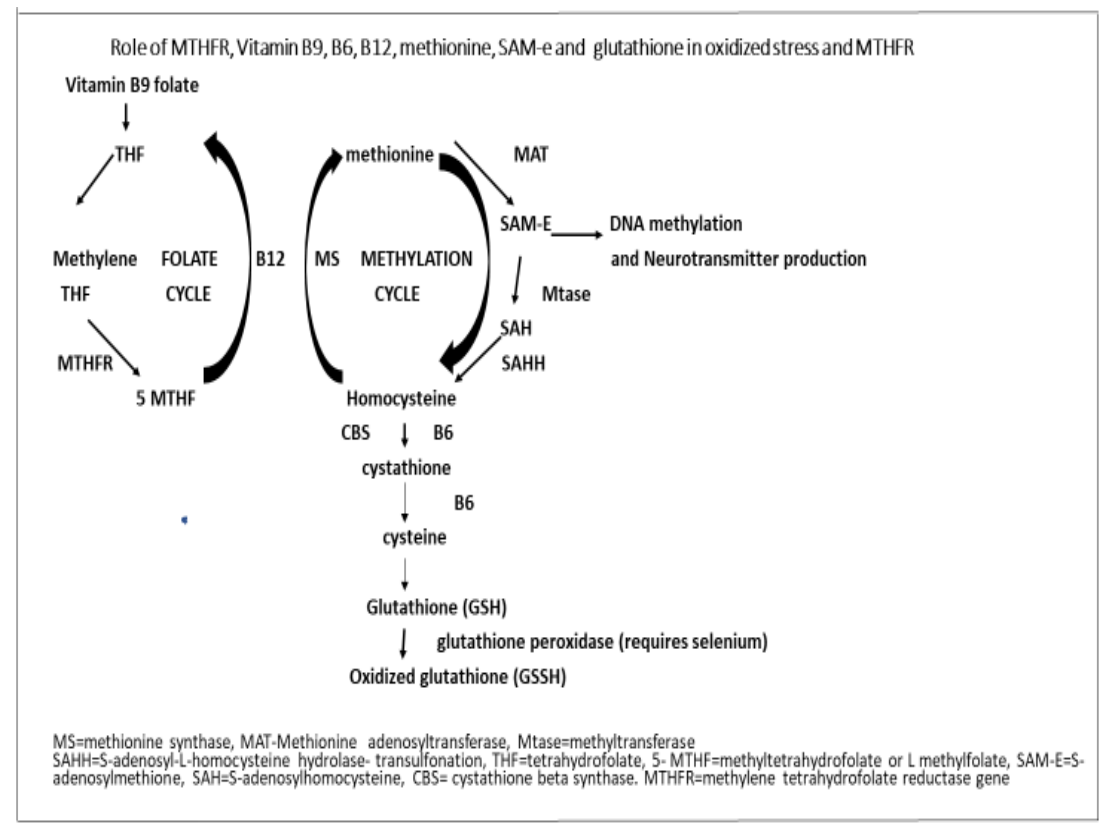

Figure 4

Thus, disturbances in the methylation cycle (by the siphoning off of glutathione to reduce oxidative stress) and in the folate cycle (by less absorption of Vitamin B12 and folate due to gut inflammation can lead to depression, which can be exacerbated by effects of the MTHFR genotype with a T allele (Figure 1).

\subsection{Lifestyle/Experience Contributions to Inflammation (Figure 5).}

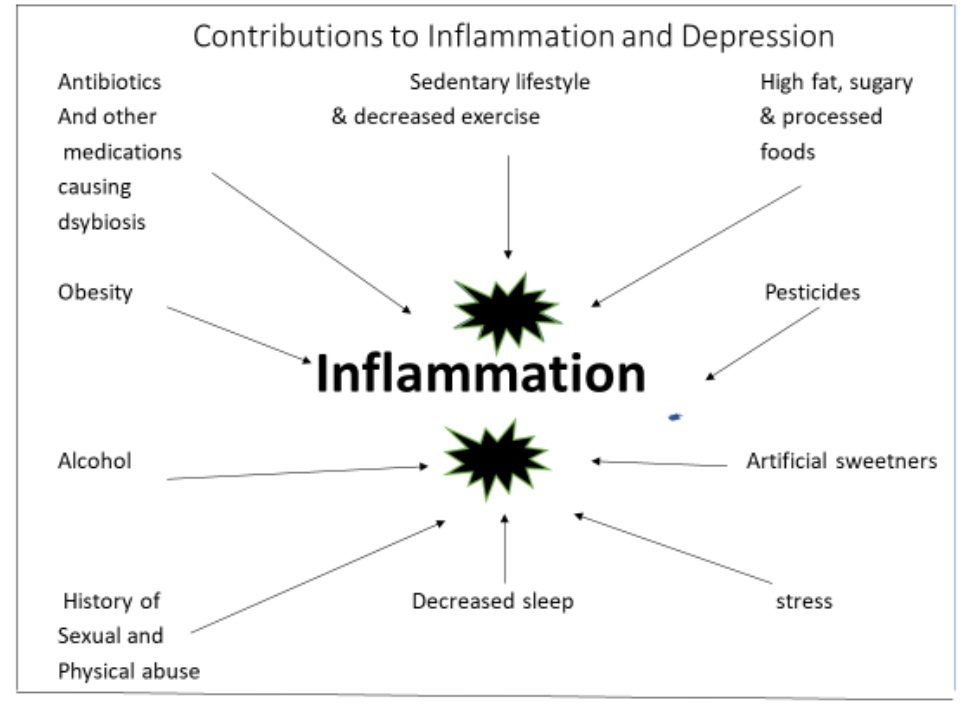

\section{Figure 5}

The usual American diet may cause dysbiosis. High-fat and high-sugar fast foods and processed food can increase LPS, leading to dysbiosis and increase of intestinal permeability and depression symptoms [33]. Processed foods, sugary beverages, sedentary lifestyle, and associated weight gain are associated with higher incidence of depression $[9,10]$. In addition, obesity from processed foods has been associated with depression [6, 11,34]. In fact, high fat foods, processed foods and sugar 
interfere with leptin and insulin signaling. Decreased leptin signaling impairs satiety and increases insulin resistance leading to obesity and subsequent depression [35]. The use of artificial sweeteners to avoid sugar are linked to glucose intolerance [29, 36], may raise blood sugar, and favoring growth of bacteria associated with obesity, like Bacteroidetes and Firmicutes [29].

However, an inflammatory diet may be indicator of risk for depression regardless of high body mass index. In a study of the effects of an inflammatory diet and depression in children, data was prospectively collected over 10 years from the Avon Longitudinal Study of Parents and Children (ALSPAC) cohort $(\mathrm{N}=6939)$ free from depression at baseline (age 8.5 years). An inflammatory pattern (IDP) score from a food frequency questionnaire was measured at 8.5 years and levels of inflammatory biomarkers, C-reactive protein and interleukin-6, at 9.5 years. At age 18 years, depression cases were diagnosed via the International Statistical Classification of Diseases, 10th Revision (ICD-10) and the Clinical Interview Schedule-Revised (CIS-R) depression score. Participants in the highest tertile of IDP score had 1.34 times the risk of developing depression compared to those in the lowest tertile $(95 \% \mathrm{Cl}, 1.08-1.66 ; P$-trend<0.01). In addition, the correlation of IDP tertiles and depression showed a marginal trend among participants who were not overweight or obese $(p<0.10)$ but not among participants who were overweight or obese [37]. Therefore, inflammatory diets may also increase the risk of depression in non-obese children. Levels of IL- 6 and CRP were measured in non-fasting blood samples obtained in participants at age 9 years in this study [38]. Participants in the top third of IL-6 values compared with the bottom third at age 9 years were more likely to be depressed (CIS-R) at age 18 years (adjusted odds ratio [OR], 1.55; 95\% Cl, 1.13-2.14). Results using the Mood and Feelings Questionnaire (MFQ) were similar. Risks of psychotic events and of psychotic disorder at age 18 years were also increased with higher IL- 6 levels at baseline (adjusted $\mathrm{OR}, 1.81 ; 95 \% \mathrm{Cl}, 1.01-3.28$; and 2.40; $95 \% \mathrm{Cl}, 0.88-6.22$, respectively). Thus, later interventions may be helpful but may not be able to remedy inflammation completely. Early intervention should be the gold standard to avoid inflammation and risk for depression and other psychiatric disorders.

The reduction in the American diet of fermented food (containing probiotics) has also decreased the bioavailability of mood-regulating B vitamins, magnesium, and zinc, (which are necessary for the production of neurotransmitters) and these deficits are linked to depression [29, 30].

Exposure to sexual and physical abuse during childhood as well as sedentary lifestyle increases the risk of severe obesity $[39,40]$. Insufficient sleep can also increase obesity by decreasing glucose tolerance, insulin sensitivity, and leptin and increasing ghrelin [41,42]. Decreased sleep can increase cortisol hyperarousal and inflammation. Adolescents and young adults (age 10-22) with insomnia symptoms and short sleep duration ( $<7$ hours) had greater plasma c-reactive protein (CRP) levels than adolescents with insomnia symptoms and normal sleep duration ( $>7$ hours) [43].

Adolescents often begin experimenting with alcohol in their teens and some become alcoholdependent. One study demonstrated that some alcohol-dependent subjects developed gut "leakiness", associated with altered composition and activity of the gut microbiota and higher scores of depression, anxiety, and alcohol craving upon three weeks of abstinence [44].

Other modern factors that may lead to production of pro-inflammatory cytokines and increased gut permeability include pesticides [12]. Slow metabolizers (e.g., cytochrome 2D6) may not be able to metabolize pesticides, allowing them to accumulate to toxic levels causing inflammation and oxidative stress. Gut bacteria could be affected by pesticides, similar to certain medications like antibiotics [13]. In fact, in a study of over 2000 cases with depression (age 15-65), treatment with a 
single antibiotic course was associated with higher risk for depression, e.g., adjusted odds ratios were 1.23 for penicillins $(95 \% \mathrm{Cl}, 1.18-1.29)$ and 1.25 (95\% Cl, 1.15-1.35) for quinolones [45]. Proton pump inhibitors (PPI) and antipsychotic medications are associated with a decrease in diversity of gut microbiome and increased susceptibility to Clostridium difficile infection [46]. One study revealed a decreased ratio of Bacteroidetes: Firmicutes in the gut microbiome shortly after chronic use of risperidone in children, with a corresponding increase in body mass index over time [47].

Thus, inflammation caused by the change in the American diet to high fat, sugary, and processed foods, obesity, sedentary lifestyle, lack of sleep, stress, a history of sexual and physical abuse, alcohol dependence, artificial sweeteners, pesticides, and medications, can all play a role in the disruption of the gut-brain axis and subsequent development of depression, and may be linked to refractory depression (See Figure 5).

\subsection{Treatment Strategies}

Obviously, simple (but hard to implement) changes in lifestyle and diet may help avoid or decrease depression.

\subsection{Treatments Addressing Food and Vitamin/Mineral Deficiencies}

Moving to fermented foods (containing probiotics) can increase the bioavailability of moodregulating $B$ vitamins. Assessing magnesium, vitamin $D$, selenium, and zinc levels and supplementing as indicated may also be helpful. A number of studies in adolescents have linked depression to deficiencies in these micronutrients.

\subsubsection{Magnesium}

Magnesium deficiency is linked to a dysregulation of the HPA axis and increased inflammation. Magnesium modulates mood by acting as an N-methyl-d-aspartate (NMDA) antagonist, supporting synthesis of serotonin, dopamine and noradrenalin, increasing Brain Derived Neurotrophic Factor (BDNF) expression, and improving the sleep-wake cycle. Magnesium is found in green leafy vegetables, some legumes, seeds, nuts, and whole grains [48]. One study of 180 pregnant adolescents found higher symptoms of depression in those with lower magnesium intake [49].

Since inadequate brain magnesium appears to reduce serotonin levels, and since antidepressants have been shown to raise brain magnesium, magnesium supplementation or eating fermented foods that increase magnesium may be beneficial for depression [50].

\subsubsection{Folate (Vitamin B9)}

Folate (B9), after its conversion to L-methyl folate) is a co-factor for biosynthesis of SAMe. The latter is involved in the production of norepinephrine, dopamine and serotonin [51] (Figure 4). Foods containing vitamin B9 include lettuce, asparagus, spinach, broccoli, and oranges [31]. A study of 26 inpatient girls admitted with depression found serum levels of docosahexaenoic acid (DHA), arachidonic acid (AA), and folate significantly lower than those in a control group [52]. A crosssectional study of over 6000 adolescents found folate intake inversely associated with depressive symptoms in both boys and girls [53]. In a study of ten adolescents with treatment-resistant depression (mean age $14.4 \pm 2.8$ years), 8 (80\%) had a single mutation among the 2-methylene 
tetrahydrofolate reductase (MTHFR) gene variants evaluated (50\% A1298 AC; 30\% C677 CT), indicating reduced MTHFR activity. All had failed 3 antidepressant trials. When the 10 were prescribed adjunctive I-methylfolate (LM), 8 (80\%) demonstrated improvement in depression, anxiety, and irritability [54].

\subsubsection{Cobalamin (Vitamin B12) (Figure 4)}

Like vitamin B9, B12 is involved in the synthesis of neurotransmitters. Considering the relationship between declining absorption of B12 and increasing depression in the elderly, it may be helpful to monitor B12 in youth with chronic malabsorption, like Celiac disease. Vitamin B12 is found in animal protein, such as, liver, salmon, yogurt and eggs [48]. A youth with severe B12 deficiency, depression, and psychotic symptoms demonstrated improvement 1-2 weeks after B12 injections [55].

\subsubsection{Pyroxidine (Vitamin B6) (Figure 4)}

Vitamin B6 is involved in the production of glutathione (Figure 2). It is found in poultry, fish, vegetables, eggs, and brown rice [48]. A cross-sectional study in 3,067 boys and 3,450 girls aged 1215 found B-6 intake was inversely associated with depressive symptoms (score $\geq 16$ on Center for Epidemiologic Studies Depression Scale) in both boys (OR $[95 \% \mathrm{Cl}], 0.73[0.54,0.98]$; $p$ for trend = .02 ) and girls (OR [95\% Cl], 0.72 [0.56, 0.92]; $p$ for trend = .002) [53].

\subsubsection{Zinc}

Zinc is a cofactor for $>100$ enzymes. Its deficiency results in increased glutamate, an excitatory molecule found in depression. Glutamate affects the HPA axis, increasing cortisol. Zinc acts as an antidepressant by antagonizing NMDA receptors and elevating BDNF in the hippocampus and cortical regions. Zinc is found in beans, nuts, red meat, oysters, crabs, lobsters, whole grains, and dairy products [48].

In a meta-analysis involving 17 studies with 1643 depressed and 804 control subjects (including young adults), peripheral blood zinc concentrations were approximately $1.85 \mu \mathrm{mol} / \mathrm{L}$ lower in depressed subjects than controls. Depression was associated with a lower concentration of zinc in peripheral blood [49]. Zinc has been used successfully for augmentation in previously treatmentresistant adults with depression and plays a role in producing neurotransmitters by helping convert dietary pyridoxine to the active form of B6, pyridoxal phosphate [50].

\subsubsection{Calciferol (Vitamin D3)}

In depression, 25(OH)D level was inversely associated with symptom severity, suggesting a doseresponse gradient, and low vitamin D carried risk of having a depressive disorder at 2-year followup [39]. Foods containing Vitamin D are eggs, liver, salmon, milk (artificially added), oysters and shrimp but most vitamin D comes from the sun [48]. Vitamin D 3 deficiency has been linked to increasing levels of pro-inflammatory cytokines and insulin resistance [30]. Vitamin D is considered a neurohormone essential to brain development, and deficiencies have been linked to depression, bipolar disorder, schizophrenia, and autism [51, 56, 57]. A prospective study assessed Vitamin D levels and a Mood and Feelings Questionnaire in 2,750 youth (mean ages 9.8, 10.6, and 13.8 years). 
Higher levels of Vitamin D at 9.8 years predicted lower depressive symptoms at 13.8 years but not at 10.6 years, suggesting that puberty brings more sensitivity to this deficiency [58]. An open label study treated 48 adolescents with clinical depression and low vitamin D levels for 3 months (4000 IU/ day for one month, then 2000 IU/day for 2 months). The youth demonstrated improvements in self-rated depression scores $(p<.05)$ on the Mood and Feelings Questionnaire-short version [59]; without a control group, it is difficult to interpret this result.

Thirty-four 18-70-year olds with DSM IV bipolar depression and Vitamin D insufficiency ( $<30$ $\mathrm{ng} / \mathrm{ml}$ ) were randomized to 5000 IU Vitamin D3/day vs. placebo for 12 weeks. Despite a greater rise in Vitamin D levels in the supplemented group, there was no significant difference in reduction of depressive symptoms. However, both groups' Vitamin D levels remained insufficient [60]. Therefore, higher dosages and/or longer treatment may have improved symptoms. In fact, a crosssectional study in adults found that the risk of depression only began to decrease when Vitamin $D$ levels were above $42 \mathrm{ng} / \mathrm{ml}$ [61]. Therefore, depression treatment may require higher levels along the line of claims that optimum levels should be between $50-80 \mathrm{ng} / \mathrm{ml}$. In addition, whether vitamin $D$ is taken with a fatty meal to promote absorption is an important clinical management issue.

\subsubsection{Selenium}

Selenium deficiency is associated with thyroid dysregulation, oxidative stress, and inflammation. Selenium is necessary for the conversion of GSH to GSSH to neutralize ROS (Figure 3). Selenium and zinc are necessary for the conversion of thyroxine (T4) to active triiodothyronine (T3). Selenium acts as an anti-depressant by modulating serotonin, dopamine and adrenaline and attenuating inflammation. Foods containing selenium include seafood, grains, meat, poultry, fish and eggs. Where the food was grown affects Se levels because of wide variation in regional soil levels [48].

More than $50 \%$ of depressed pregnant teenagers had an inadequate intake (excluding dietary supplement) of folate, vitamin A, vitamin E, iron, zinc, calcium, magnesium, and phosphorous. Additionally, $>20 \%$ of participants had an inadequate intake of thiamin, riboflavin, niacin, vitamin B6, vitamin B12, vitamin C, copper, and selenium [49].

In summary, Zinc, magnesium, B-vitamin, selenium, and Vitamin-D deficiencies should be considered in depressed youth. Blood levels should be monitored in unresponsive cases. Also, measuring deficits in the folate cycle like folate, vitamin B12 or methylmalonic acid (an early indicator of B12 deficiency), and checking MTHFR genotype may be helpful. Measuring deficiencies in the methylation cycle may also be helpful, which would include homocysteine, SAM-E and methylmalonic acid (Figure 3). A broadspectrum micronutrient supplement may be the most practical way to address the majority of the deficiencies/insufficiencies.

\subsection{Treatment for Oxidative Stress}

Lower levels of total and reduced glutathione (GSH) have been associated with later onset of bipolar disorder (BD). Although a low GSH/GSSH ratio level may be indicative of oxidative stress, a better indicator of oxidative stress, which damages mitochondria, may be serum superoxide dismutase (SOD). An increase in SOD, which neutralizes ROS found in oxidative stress, has been associated with BD [27]. SOD was significantly increased $(p<0.001)$ in manic and depressed BD compared to either controls or euthymic BD patients. The superoxide dismutase increase may be a compensatory mechanism in the acute phase of mania when oxidative stress is at its highest [62]. 


\subsection{N-Acetylcysteine (Nac)}

Administration of NAC, a cysteine prodrug, replenishes intracellular GSH levels. NAC is best known for its ability to counter acetaminophen (Tylenol) toxicity, and is a safe, well-tolerated antidote for cysteine/GSH deficiency. NAC is known to decrease oxidative stress by increasing glutathione and it modulates glutamate [63]. Oxidative stress and glutamate transmission are involved in both depression and Cannabis Use Disorder (CUD). Secondary analyses in an 8-week randomized controlled trial of $\mathrm{N}$-acetylcysteine (NAC) for cannabis cessation found that the 74 adolescents with baseline depressive symptoms had significantly fewer positive urine cannabinoid tests than those without depressive symptoms. [64]. (see Figure 3). Perhaps decreasing oxidative stress indirectly influenced depression which may have decreased the urge to self-medicate with cannabis. In an RCT of adult bipolar patients 1000 mg of NAC twice a day resulted in a large decrease in depressive symptoms compared to the placebo group [65]. NAC seems to do more for BD depression than for mania.

\subsection{Treatment to Address Inflammation}

\subsubsection{Essential Fatty Acids}

Both omega-6 $(\Omega 6)$ and omega-3 $(\Omega 3)$ fatty acids are considered essential; they are precursors of pro-inflammatory and anti-inflammatory eicosanoids (prostaglandins, leukotrienes, thromoboxanes), respectively. In the $\Omega 3$ series, eicosapentaenoic acid (EPA) provides antiinflammatory cell signalers while docosahexaenoic acid (DHA) (and to some extent EPA) is needed for neuronal membranes. The balance of $\Omega 6: \Omega 3$ provides a "supply side" regulation of inflammation. Youth with mood disorders have higher ratios of omega- $6(\Omega 6)$ to omega- 3 fatty acids $(\Omega 3)$ than those without mood disorders. In a seven-year follow-up of youth with a high risk for psychosis, a higher $\Omega 6: \Omega 3$ ratio at baseline predicted later mood disorders but not other psychiatric disorders [66].

A randomized pilot trial $(N=23)$ in 5-12-year old children found greater reduction of depressive and manic symptoms with a combination of $\Omega 3$ fatty acids and inositol than with inositol alone [67]. In 28 children with major depression, $70 \%$ of those receiving $\Omega 3$ at a 2:1 ratio of EPA:DHA [380-400 mg EPA; $180-200 \mathrm{mg}$ DHA) but none receiving placebo enjoyed greater than $50 \%$ reduction in depressive symptoms. In a randomized comparison of $\Omega 6$ to $\Omega 3$ supplementation for depression in 11-17-year-olds, only omega-3 showed significant improvement, and this was confined to those with major depression rather than mixed anxiety \& depression [68].

In the Omega-3 and Therapy Studies (OATS), 23 youth age 8-14 with bipolar disorder NOS and 72 with depression were assigned in a $2 X 2$ design to $\Omega 3(1.6 \mathrm{~g} /$ day of EPA+DHA), psychoeducational psychotherapy (PEP) plus placebo matched to $\Omega 3, \Omega 3$ plus PEP, or placebo. In the bipolar study $\Omega 3$ showed more benefit for depression than placebo, both alone $(d=.48)$ and combined with psychoeducational psychotherapy (PEP) $(d=1.7, p=.01)$ [69]. In the depression study, $\Omega 3$ alone ( $d$ $=.42)$ and combined with PEP $(\mathrm{d}=.28)$ surpassed placebo in relief of depressive symptoms, but not significantly. However, fewer psychosocial stressors significantly moderated a more favorable depression response to $\Omega 3$, suggesting value for more biologically based depression rather than reactive depression. [70]. Also, those with higher $B M I$ benefitted significantly more from $\Omega 3$ compared to placebo than those with lower BMI [71]. In the same two studies, an analysis of the 
pooled samples found significantly greater $(p=0.001, d=0.70)$ improvement with $\Omega 3$ than placebo on a parent-rated composite scale of executive function [72].

Considering all available data, supplementation with up to a gram per day of mercury-free EPA+DHA seems advisable in treating child or adolescent depression. For safety and optimal utilization, antioxidant vitamins/minerals (possibly as broad spectrum micronutrients) should be given concomitantly.

\subsubsection{Probiotics}

Probiotic treatment has been shown to reduce the hyperactivity of the HPA axis [73]. In an RCT 66 patients hospitalized with mania were adjunctively given either Lactobacillus rhamnosus strain GG and Bifidobacterium animalis subsp. lactis strain Bb12 or placebo in a parallel two-group design. During the 24-week observation period there were a total of 24 rehospitalizations in the 33 individuals who received placebo and eight rehospitalizations in the 33 individuals who received the probiotics ( $z=2.63, P=.009$ ) [74]. Another study, [75] found that probiotic formulations of Bifidobacterium longum and Lactobacillus helveticus significantly decreased anxiety in humans. In a double-blind RCT [76] 40 adults with a DSM-IV diagnosis of MDD age 20-55 had significant beneficial effects on Beck Depression Inventory, insulin resistance, hs-CRP concentrations, and glutathione concentrations with Lactobacillus acidophilus, $2 \times 10$ (9) Colony-Forming Units (CFU)/g, Lactobacillus casei $2 \times 10$ (9) CFU/g), and Bifidobacterium bifidum $2 \times 10$ (9) CFU/g for 8 weeks.

Combining probiotics with prebiotics seems even more beneficial. Prebiotics can lower LPS and increase satiety peptides (thus decreasing hunger and metabolic parameters associated with obesity) [77]. Probiotics (Lactobacilli and Bifidobacterium) with prebiotics improved body weight, abdominal fat, and intestinal barrier function [78]. In a randomized trial [79] 110 depressed patients were randomly assigned to receive the probiotic (Lactobacillus helveticus and Bifidobacterium longum), prebiotic (galactooligosaccharide), or placebo for 8 weeks. Eighty one patients completed the study. Probiotic supplementation resulted in a significant decrease in BDI score (17.39 to 9.1) compared to the placebo (18.18 to 15.55$)$ and prebiotic (19.72 to 14.14$)$ supplementation $(p=$ 0.042 ). The kynurenine/tryptophan ratio (an indicator of the IDO pathway) decreased significantly in the probiotic group compared to placebo after adjusting for serum isoleucine $(p=0.048)$.

Probiotics may decrease depression by decreasing inflammation and oxidative stress. In a metaanalysis probiotics supplementation resulted in significant reduction in the Hamilton Depression Rating Scale (HAMD) and a reduction in C-reactive protein, IL-10, and malondialdehyde (an indicator of oxidative stress). However, changes in the Beck Depression Inventory, TNF- $\alpha$, IL-6, nitric oxide (indicator of free radicals), glutathione, and total antioxidant capacity [80] were not significant.

Early interventions to change the course of dysbiosis may be helpful. Probiotic feeding during the early years modulated the gut microbiota 10 years later, which restrained excessive weight gain [81]. In a RCT, 75 infants were randomized to Lactobacillus rhamnosus or placebo during the first 6 months of life and were followed for 13 years. Gut microbiota was assessed at the ages of 3 wk, 3, $6,12,18,24 \mathrm{mo}$, and 13 years. ADHD or autism was diagnosed in $6 / 35$ (17.1\%) of children in the placebo group and none in the probiotic group $(P=0.008)$. The mean $(S D)$ numbers of Bifidobacterium species bacteria in feces had been lower during the first 6 mo of life in affected children 8.26 (1.24) log cells/g than in healthy children $9.12(0.64) \log$ cells/g; $P=0.03$. Probiotics fed to infants decreased intestinal permeability as measured by lactulose/mannitol ratio [82]. 
Prospective larger studies are needed to determine if early use of probiotics may alleviate the risk of developing depression associated with "leaky gut".

\subsection{Supplements (Other Than Vitamins or Minerals) and Lifestyle Interventions that May Affect Inflammation, the Methylation Cycle, Folate Cycle and Cortisol Levels}

\subsubsection{Saffron}

Saffron has effects similar to anti-depressants via serotonin effects. It also has anti-inflammatory and anti-oxidant effects. In a meta-analysis, consumption of saffron resulted in a significant reduction in Beck Depression Inventory (BDI) (11 studies --weighted mean difference (WMD): -4.86; 95\% Cl: -6.58, -3.14), Beck Anxiety Inventory (BAI) (5 studies) (WMD: -5.29; $95 \% \mathrm{Cl}:-8.27,-2.31$ ) and Pittsburgh Sleep Quality Index (PSQI) scores (3 studies) (WMD: -2.22; 95 \% Cl: -2.73, -1.72). It did not decrease C-reactive protein levels [83].

\subsubsection{Adaptogens}

Adaptogens are herbs used in Ayurvedic and Chinese medicine. They reduce oxidative stress by reducing the levels of nitrous oxide and help to normalize an upregulated HPA axis by unblocking the glucocorticoid receptor so that the normal homeostatic feedback process can occur [84].

In a RCT Eleutherococcus senticosus and lithium were compared to fluoxetine and lithium in 76 adolescents ages $12-17$ with bipolar depression. Remission rates $(51.4 \%$ vs $48.7 \%$ and response rates (67.6\% vs $71.8 \%$ ) were comparable for E senticosus vs fluoxetine. Three of the adolescents on fluoxetine experienced a manic switch [85].

\subsubsection{St John's Wort (hypericum; SJW)}

St. John's Wort is known to modulate neurotransmitter levels and their receptors, including serotonin, norepinephrine, dopamine, $\gamma$-aminobutyric acid, and glutamate; modify inflammatory cytokines; inhibit cortisol production; modulate neuronal ionic conductance; elevate intracellular sodium concentration; and induce neurogenesis and neuroprotection. However, in the U.S. it is known to contain highly varied amounts of the active ingredient hypericum [86]. Batch-to-batch variability complicates reliable effects.

In an open trial [87] 33 youth with moderate to severe mood disorder (Child Depression Rating Scale-Revised (CDRS-R) >40) took 150-300 mg 3 times/day. Response criteria (CDRS-R $<28$ ) were met by $24 \%$ by week 1 and $83 \%$ by week 8 . Another open label trial [88] using $300 \mathrm{mg} 3$ times/day had similar results but there was a $57 \%$ drop out rate due to side effects.

Without a control group, the results are hard to interpret but the rapid response rate and positive results suggests more studies are needed. However, clinicians should be aware that St John's Wort can cause serotonin syndrome, discontinuation syndrome, and sunlight sensitivity and induce cytochrome P450 isoenzymes CYP 3A4, 2C19 and 2C9. There is also concern that at high dosages it may cause seizures and mania [89].

\subsubsection{S-adenosylmethionine (SAMe)}

Due to the fact that SAMe is difficult to synthesize and breaks down easily when exposed to water, some formulations may not have detectable levels. SAMe plays a role in the methylation 
cycle and is involved in neurotransmitter production (See Figure 3). An open trial in 3 youth with clinical depression using 600-1400 mg/day found all had a rapid response that persisted for 22 weeks [90]. Clinicians should be aware that it can have the same effects as tricyclics and psychostimulants and can induce mania. It needs further study.

\subsubsection{Uridine}

Uridine is a pyrimidine nucleoside, thought to impact glutamate. Pyrimidines are thought to have beneficial effects on cerebral phospholipid metabolism, catecholamine synthesis, and mitochondrial function all of which have been linked to depression Seven bipolar adolescents treated with $500 \mathrm{mg} / \mathrm{d}$ for 6 weeks had significant decreases on the CDRS, from 65.6 to 27.2 [91]. It needs better study.

\subsubsection{Meditation}

Meditation may help normalize the hyperactivation of the HPA axis by decreasing vagal nerve stimulation [92]. In a study using yoga and meditation for 3 months there were increases in antiinflammatory cytokines like IL-10, decreases in the pro-inflammatory cytokine Interleukin-12, increases in Brain Derived Neurotrophic Factor (BDNF). BDNF is a key neurotrophin that promotes development, survival and plasticity of neurons in the central and peripheral nervous systems. There were clearly significant improvements in the Brief Symptom Inventory-18 scores, including the total score $(t .33 /=4.66, p<0.0001)$ as well as each of the subscores which included depressive (t.33/ = 2.84, p < 0.01), anxious (t.33/ = 4.22, p < 0.0001), and somatic (t.33/ = 4.66, p < 0.0001) symptoms [93]. In a study of 58 individuals (age 19-50 years old) with Major Depression diagnosed with DSM-5 criteria, participants were randomly assigned to Yoga and meditation or a control group for 12 weeks. There was a significant decrease [difference between means, $(95 \% \mathrm{Cl})$ ] in Beck Depression Index-II score change in value, time 1 and time $2[-5.83(-7.27,-4.39), p<0.001]$ and significant increase in BDNF (ng/ml) $[5.48(3.50,7.46), \mathrm{p}<0.001]$ after Yoga Meditation Lifestyle Intervention (YMLI) compared to control group. Among the mind-body communicative biomarkers, there was a significant increase in circulating dehydroepiandrosterone (DHEAS-an indication of reduced inflammation) and sirtuin 1 (used in repair of DNA) and a significant decrease in circulating cortisol and IL-6 (a pro-inflammatory cytokine) after YMLI compared to yoga group at baseline level and control group. A total of 12 weeks YMLI showed improvement in cellular health biomarkers that included - significant decrease in 8 Oxo-2'-deoxyguanosine (8OH2dG- a marker of DNA damage); significant increase in total anti-oxidant capacity (TAC) and decrease in ROS (markers of oxidative stress); and significant increase in telomerase activity (marker of telomere attrition) compared to control group (all $p<0$. 05) (Table 3). However, change in telomere length was not significant in both groups. Also, the control group showed significantly increased ROS and IL-6 levels compared to baseline $(P<0.001)$. As noted earlier an overactive HPA and increased cortisol is associated with depression. In this study, increased cortisol was associated with reductions in BDNF and increase in depression severity [94].

\subsubsection{Exercise}

Exercise can increase Peroxisome Proliferation Activated Receptor Gamma Coactivator 1 (PGC1 alpha) gene expression, which reduces synthesis and release of pro-inflammatory cytokines and 
decreases glutamatergic neurotoxicity [95]. The Depressed Adolescents Treated with Exercise (DATE) study randomized 30 non-medicated adolescents age 12-18 with DSM-IV-TR major depressive disorder. Thirty adolescents aged 12-18 years were randomized to either vigorous exercise (EXER) (>12 kg/kcal/week [KKW]) or a control stretching activity (< $4 \mathrm{KKW})$ for 12 weeks. By week 12 , the exercise group had a $100 \%$ response rate ( $86 \%$ remission), whereas the stretch group response rate was $67 \%$ (50\% remission) $(p=.02)$ [96].

\section{Discussion/Conclusions}

$\mathrm{CIM}$ interventions are appealing to parents and youth because of the perception that they are safer and will avoid stigma and possibly the need to see a psychiatrist regularly. However, they are not without risks, especially if parents or adolescents do not alert a clinician about use of over -thecounter supplements. There are many drug-supplement interactions. Most supplements and herbs have not been reviewed by the FDA before marketing and do not have FDA-approved good manufacturing procedures in place. Products may vary in amounts of active ingredients, and patients often take supplements or herbs without knowing established recommended dosages. The purity of supplements has been examined by Consumerlabs.Com, where clinicians may obtain more information. However, clinicians must be aware that some companies pay sites to recommend their products. Clinicians need to be aware of patient use of supplements and expand their knowledge of commonly used CIM treatments, keeping in mind that herbs, if they work, are crude drugs without good quality control.

Research increasingly recognizes that there may be abnormalities and individual variations in biological systems involving the gut-brain axis, inflammation, oxidative stress, folate and methylation cycles and cortisol levels that prevent conventional established interventions from being universally effective. CIM research, although in its infancy, is beginning to recognize that certain supplements and other interventions used adjunctively, targeting systems associated with these abnormalities, may decrease depressive symptoms. Addressing problems with inflammation in the gut, oxidative stress, mineral and vitamin deficiencies, diet, abnormalities in the folate and methylation cycles, sleep, sedentary lifestyle, obesity, and stress may help reduce the severity of depression, particularly if conventional interventions have not worked. Avoiding medications that disrupt the gut microbiome or that decrease B12 and CoQ 10 (like metformin) or proton pump inhibitors (which decrease B12) may reduce the severity of depression. When such drugs cannot be avoided, prophylactic B12 supplement should be considered. Using supplements like St. John's Wort (to increase neurotransmitters, decrease cortisol, and modify inflammatory cytokines), adaptogens (which allow the HPA axis to normalize and reduce oxidative stress), and saffron (which affects serotonin and acts as an anti-oxidant and anti-inflammatory) may act as adjuncts in the treatment of refractory depression. The best interventions seem to include the addition of omega 3 fatty acids. Normalizing the gut with probiotics may also prove to be useful. Much more research is needed. One thing is clear: conventional interventions do not always work, and when they don't, other interventions should be considered.

Although it is imperative to decrease inflammation, once the inflammatory process becomes severe nutritional interventions may no longer be effective. E.g., In a RCT of 1950 patients, a protocol-guided individualized nutritional support program was compared to a standard hospital food control group. Baseline measurement of C-reactive protein (CRP) divided the sample into low, 
moderate and high inflammation ( $<10 \mathrm{mg} / \mathrm{L}, 10-100 \mathrm{mg} / \mathrm{L}$ and $>100 \mathrm{mg} / \mathrm{l}$, respectively). Compared to the control group, patients receiving nutritional support showed a significant reduction in 30-day mortality except the subgroup with high baseline inflammation, which showed no benefit of nutritional support [97]. Therefore, waiting to treat after inflammation occurs may lose efficacy once inflammation becomes severe.

While awaiting further research, there are some things clinicians can reasonably apply now. Figure 6 and 7 suggests a therapeutic decision-flow algorithm reflecting the current state of knowledge.

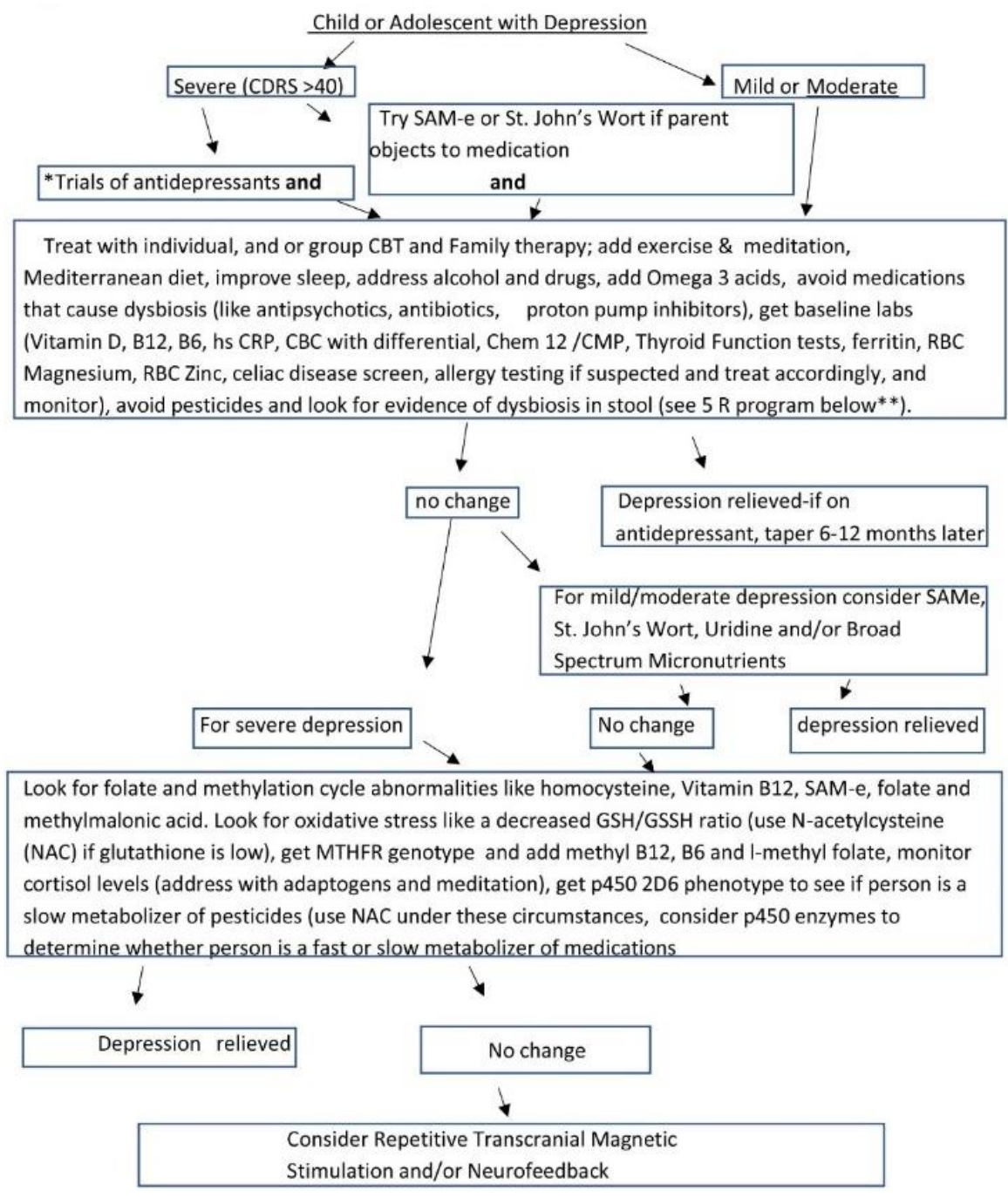

Figure 6 
** 5 R's [98]

1. Remove-: allergic foods, parasites or other bad bugs such as bacteria or yeast. This might involve using an "elimination diet" to pick up sensitivities to certain foods

2. Replace: add back things like digestive enzymes, hydrochloric acid and bile acids that are required for proper digestion.

3. Reinoculate: Use probiotic foods or supplements that contain

bifidobacteria and lactobacillus species, and use high-soluble-fiber foods or prebiotic supplement that increases short chain fatty acids (SCFA) and maintains gut integrity.

4. Repair: Help repair the gut by adding key nutrients (like zinc) or antioxidants (like vitamins A, C, and E), L- glutamine and omega 3 fatty acids if not already added.

5. Rebalance: Pay attention to lifestyle choices - sleep, exercise and stress can all affect the Gl tract. (See Figure 2)

Figure 7 [98]

\section{Author Contributions}

Dr. Simkin was the author and Dr. Arnold was the co-author.

\section{Funding}

Dr. Simkin has no disclosures.

Dr. Arnold has received research funding from Curemark, Forest, Lilly, Neuropharm, Novartis, Noven, Shire, Supernus, Roche, Otsuka, and Young Living (as well as NIH and Autism Speaks), has consulted with Gowlings, Neuropharm, Organon, Pfizer, Sigma Tau, Shire, Tris Pharma, and Waypoint, and been on advisory boards for Arbor, Ironshore, Novartis, Noven, Otsuka, Pfizer, Roche, Seaside Therapeutics, Sigma Tau, Shire.

\section{Competing Interests}

The authors have declared that no competing interests exist.

\section{References}

1. Mojtabai R, Olfson M, Han B. National trends in the prevalence and treatment of depression in adolescents and young adults. Pediatrics. 2016; 138: e 20161878

2. Lara GA, Zuniga JO, Perez OC, Solís SH, Jiménez CE, Méndez MC. Predictors of suicidal ideation and depressive symptoms in Chiapas, Mexico. Cien Saude Colet. 2018; 23: 1089-1096.

3. Twenge JM, Joiner TE, Rogers ML, Martin GN. Increases in depressive symptoms, suiciderelated outcomes, and suicide rates among U.S. Adolescents after 2010 and links to new media screen time. Clin Psychol Sci. 2018; 6: 13-17.

4. Cipriani A, Zhou X, Del Giovane C, Hetrick SE, Qin B, Whittington C, et al. Comparative efficacy and tolerability of antidepressants for major depressive disorder in children and adolescents: $A$ network meta-analysis. Lancet. 2016; 388: 881-890. 
5. Ignaszewski MJ, Waslick B. Update on randomized placebo-controlled trials in the past decade for treatment of major depressive disorder in child and adolescent patients: A systematic review. J Child Adol Psychop. 2018; 28: 668-675.

6. Foster JA, Rinaman L, Cryan JF. Stress \& the gut-brain axis: Regulation by the microbiome. Neurobiol Stress. 2017; 7: 124-136.

7. Simkin DR. Microbiome and mental health, specifically as it relates to adolescents. Currt Psychiatry Rep. 2019; 21: 93.

8. Scaini G, Rezin GT, Carvalho AF, Streck EL, Berk M, Quevedo J. Mitochondrial dysfunction in bipolar disorder: Evidence, pathophysiology and translational implications. Neurosci Biobehav Rev. 2016; 68: 694-713.

9. Moreira AP, Texeira TF, Ferreira AB, Peluzio MD, Alfenas RD. Influence of a high-fat diet on gut microbiota, intestinal permeability and metabolic endotoxaemia. Br J Nutr. 2012; 108: 801-809.

10. Malik VS, Willett WC, Hu FB. Sugar-sweetened beverages and BMI in children and adolescents: Re-analyses of a meta-analysis. Am J Clin Nutr. 2009; 89: 438-439.

11. Mannan M, Mamun A, Doi S, Clavarino A. Prospective associations between depression and obesity for adolescent males and females-a systemic review and meta-analysis of longitudinal studies. PloS One. 2016; 11: e0157240

12. Singh NK, Banerjee BD, Bale K, Basu M, Chhillar N. Polymorphism in cytochrome P450 2D6, glutathione S-transferases Pi 1 genes, and organochlorine pesticides in Alzheimer disease: A case-control study in north Indian population. J Geriatr Psychiatry Neurol. 2014; 27: 119-127.

13. Reijnders D, Goossens GH, Hermes GD, Neis EP, Van der Beek CM, Most J, et al. Effects of gut microbiota manipulation by antibiotics on host metabolism in obese humans: A randomized double-blind placebo-controlled trial. Cell Metab. 2016; 24: 63-74.

14. Kelly JR, Kennedy PJ, Cryan JF, Dinan TG, Clarke G, Hyland NP, et al. Breaking down the barriers: The gut microbiome, intestinal permeability and stress-related psychiatric disorders. Front Cell Neurosci. 2015; 9: 392.

15. Bonaz B, Bazin T, Pellissier S. The vagus nerve at the Interface of the microbiota-gut-brain axis. Front Neurosci. 2018; 12: 49.

16. Lyte M, Bailey MT. Neuroendocrine-bacterial interactions in a neuro toxin induced model of trauma. J Surg Res. 1997; 70: 195-201.

17. Karlović D, Serretti A, Vrkić N, Martinac M, Marčinko D. Serum concentrations of CRP, IL-6, TNF$\alpha$ and cortisol in major depressive disorder with melancholic or atypical features. Psychiatry Res. 2012; 198: 74-80.

18. Plöge S, Stumpff F, Penner GB, Schulzke JD, Gäbel G, Martens H. Microbial butyrate and its role for barrier functioning in the gastrointestinal tract. Ann N Y Acad Sci. 2012; 1258: 52-59.

19. Freed RD, Mehra LM, Laor D, Patel M, Alonso CM, Kim-Schulze S, et al. Anhedonia as a clinical correlate of Inflammation in psychiatric disorders. World J Biol Psychiatry. 2019; 20: 712-722.

20. Stroud CB, Vrshek-Shallhorn S, Norkett EM, Doane LD. The cortisol awakening response (CAR) interacts with acute interpersonal stress to prospectively predict depressive symptoms among early adolescent girls. Psychoneuroendocrinology. 2019; 107: 9-18.

21. Maes M, Berk M, Goehler L, Song C, Anderson G, Gałecki P, et al. Depression and sickness behavior are Janus-faced responses to shared inflammatory pathways. BMC Med. 2012; 10: 66. 
22. Bi $W$, Zhu L, Jing $X$, Zeng $Z$, Liang $Y, X u A$, et al. Rifampicin improves neuronal apoptosis in LPSstimulated co-cultured BV2 cells through inhibition of the TLR-4 pathway. Mol Med Rep. 2014; 10: 1793-1799.

23. DiSabato DJ, Quan N, Godbout JP. Neuroinflammation: The devil is in the details. J Neurochem. 2016; 139: 136-153.

24. Barreto FS, Chaves Filho AJ, de Araújo MC, de Moraes MO, de Moraes ME, Maes M, et al. Tryptophan catabolites along the indoleamine 2,3-dioxygenase pathway as a biological link between depression and cancer. Behav Pharmacol. 2018; 29: 165-180.

25. Swardfager W, Herrmann N, Dowlati Y, Oh PI, Kiss A, Walker SE, et al. Indoleamine 2,3dioxygenase activation and depressive symptoms in patients with coronary artery disease. Psychoneuroendocrinology. 2009; 34: 1560-1566.

26. Murphy MP. Mitochondrial thiols in antioxidant protection and redox signaling: Distinct roles for glutathionylation and other thiol modifications. Antioxid Redox Signal. 2011; 16: 476-495.

27. Rosa AR, Singh N, Whitaker E, De Brito M, Lewis AM, Vieta E, et al. Altered plasma glutathione levels in bipolar disorder indicates higher oxidative stress; a possible risk factor for illness onset despite normal brain-derived neurotrophic factor (BDNF) levels. Psychol Med. 2014; 44: 24091418.

28. Rezin GT, Amboni G, Zugno Al, Quevedo J, Streck EL. Mitochondrial dysfunction and psychiatric disorders. Neurochem Res. 2009; 34: 1021.

29. Shell ER. Artificial sweeteners get a gut check. Sci Am. 2015; 31: 32-34.

30. Milaneschi Y, Hoogendijk W, Lips P, Heijboer AC, Schoevers R, Van Hemert AM, et al. The association between low vitamin D and depressive disorders. Mol Psychiatry. 2014; 19: 444451.

31. Saraswathy KN, Ansari SN, Kaur G. Association of vitamin B12 mediated hyperhomocysteinemia with depression and anxiety disorder: A cross-sectional study among Bhil indigenous population of India. Clin Nutr ESPEN. 2019; 30: 199-203.

32. Lo A, Bockting CL, Koeter MW, Snieder H, Assies J, Mocking RJ, et al. Interaction between MTHFR C677T polymorphism and traumatic childhood events predicits depression. Tranl Psychiatry. 2013; 3: e288.

33. Sánchez-Villegas A, Toledo E, De Irala J, Ruiz-Canela M, Pla-Vidal J, Martínez-González MA. Fastfood and commercial baked goods consumption and the risk of depression. Public Health Nutr. 2012; 15: 424-432.

34. Costa CS, Del-Ponte B, Assuncao MC, Santos IS. Consumption of ultra-processed foods and body fat during childhood and adolescence: A systematic review. Public Health Nutr. 2018; 21: 148159.

35. Mobbs CV, Isoda F, Makimura H, Mastaitis J, Mizuno T, Shu IW, et al. Impaired glucose signaling as a cause of obesity and the metabolic syndrome: The glucoadipostatic hypothesis. Physiol Behav. 2005; 85: 3-23.

36. Suez J, Korem T, Zeevi D, Zilberman-Schapira G, Thaiss CA, Maza O, et al. Artificial sweeteners induce glucose intolerance by altering the gut microbiota. Nature. 2014; 514: 181-186.

37. Cong X, Tracy M, Edmunds LS, Hosler AS, Appleton AA. The relationship between inflammatory dietary pattern in childhood and depression in early adulthood. Brain Behav Immun Health. 2020; 2: 100017. 
38. Khandaker GM, Pearson RM, Zammit S, Lewis G, Jones PB. Association of serum interleukin 6 and C-reactive protein in childhood with depression and psychosis in young adult life: $A$ population-based longitudinal study. JAMA Psychiatry. 2014; 71: 1121-1128.

39. Richardson AS, Dietz WH, Gordon-Larsen P. The association between childhood sexual and physical abuse with incident adult severe obesity across 13 years of the national longitudinal study of adolescent health. Pediatr Obes. 2014; 9: 351-361.

40. Strasburger VC. Children, adolescents, obesity, and the media. Pediatrics. 2011; 128: 201-208.

41. Fatima Y, Doi SA, Mamun AA. Longitudinal impact of sleep on overweight and obesity in children and adolescents: A systematic review and bias-adjusted meta-analysis. Obes Rev. 2015; 16: 137-149.

42. Pan W, Kastin AJ. Leptin: A biomarker for sleep disorders? Sleep Med Rev. 2014; 18: 283-290

43. Fernandez-Mendoz J, Li Y, Vgontza A, Fang J, Gaines J, Calhoun SL, et al. Insomnia is associated with cortical hyperarousal as early as adolescence. Sleep. 2016; 39: 1029-1036.

44. Leclercq S, Matamoros S, Cani PD, Neyrinck AM, Jamar F, Stärkel P, et al. Intestinal permeability, gut-bacterial dysbiosis, and behavioral markers of alcohol-dependence severity. Proc Natl Acad Sci U S A. 2014; 111: E4485-4493.

45. Lurie I, Yang YX, Haynes K, Mamtani R, Boursi B. Antibiotic exposure and the risk for depression, anxiety, or psychosis: A nested case-control study. J Clin Psychiatry. 2015; 76: 1522-1528.

46. Le Bastard Q, Al-Gawith GA, Grégoire M, Chapelet G, Javaudin F, Dailly E, et al. Systematic review: Human gut dysbiosis induced by non-antibiotic prescription medications. Aliment Pharmakoi Ther. 2018; 47: 332-345.

47. Bahr SM, Tyler BC, Wooldridge N, Butcher BD, Burns TL, Teesch LM, et al. Use of the secondgeneration antipsychotic, risperidone, and secondary weight gain are associated with an altered gut microbiota in children. Trans Psychiatry. 2015; 5: e652.

48. Wang J, Um P, Dickerman BA, Liu J. Zinc, selenium, and depression: A review of the evidence, potential mechanisms and implications. Nutrients. 2018; 10: 584.

49. Singh A, Trumpff C, Genkinger J, Davis A, Spann M, Werner E, et al. Micronutrient dietary intake in Latina pregnant adolescents and its association with level of depression, stress, and social support. Nutrients. 2017; 9: 1212.

50. Eby GA 3rd, Eby KL. Magnesium for treatment-resistant depression: A review and hypothesis. Med Hypotheses. 2010; 74: 649-660.

51. Popper CW. Single-micronutrient and broad-spectrum micronutrient approaches for treating mood disorders in youth and adults. Child Adolesc Psychiatr Clin N Am. 2014; 23: 591-672.

52. Tsuchimine S, Saito M, Kaneko S, Yasui-Furukori N. Decreased serum levels of polyunsaturated fatty acids and folate, but not brain-derived neurotrophic factor, in childhood and adolescent females with depression. Psychiatry Res. 2015; 225: 187-190.

53. Murakami K, Miyake Y, Sasaki S, Tanaka K, Arakawa M. Dietary folate, riboflavin, vitamin B-6, and vitamin B-12 and depressive symptoms in early adolescence: The Ryukyus child health study. Psychosom Med. 2010; 72: 763-768.

54. Dartois LL, Stutzman DL, Morrow M. L-methylfolate augmentation to antidepressants for adolescents with treatment-resistant depression: A case series. J Child Adolesc Psychopharmacol. 2019; 29: 386-391. 
55. Tufan AE, Bilici R, Usta G, Erdoğan A. Mood disorder with mixed, psychotic features due to vitamin b12 deficiency in an adolescent: Case report. Child Adolesc Psychiatry Ment Health 2012; 6: 25.

56. Bell DS. Protean manifestations of vitamin D deficiency, part 2: Deficiency and its association with autoimmune disease, cancer, infection, asthma, dermopathies, insulin resistance, and type 2 diabetes. South Med J. 2011; 104: 335-339.

57. Gracious BL, Finucane TL, Friedman-Campbell M, Messing S, Parkhurst MN. Vitamin D deficiency and psychotic features in mentally ill adolescents: A cross-sectional study. BMC Psychiatry. 2012; 12: 38.

58. Tolppanen AM, Sayers A, Fraser WD, Lewis G, Zammit S, Lawlor DA. The association of serum 25-hydroxyvitamin D3 and D2 with depressive symptoms in childhood-A prospective cohort study. J Child Psychol Psychiatry. 2012; 53: 757-766.

59. Högberg G, Gustafsson SA, Hällström T, Gustafsson T, Klawitter B, Petersson M. Depressed adolescents in a case-series were low in vitamin $D$ and depression was ameliorated by vitamin D supplementation. Acta Paediatr. 2012; 101: 779-783.

60. Marsh WK, Penny JL, Rothschild AJ. Vitamin D supplementation in bipolar depression: A double blind placebo controlled trial. J Psychiatr Res. 2017; 95: 48-53.

61. Jaddou HY, Batieha AM, Khader YS, Kanaan SH, El-Khateeb MS, Ajlouni KM. Depression is associated with low levels of 25-hydroxyvitamin D among Jordanian adults: Results from a national population survey. Eur Arch Psychiatry Clin Neurosci. 2012; 262: 321-327.

62. Kunz M, Severino C, Andreazza AC, Salvador M, Ceresér KM, Gomes FA, et al. Elevated serum superoxide dismutase and thiobarbituric acid reactive substances in different phases of bipolar disorder and in schizophrenia. Prog Neuropsychopharmacol Biol Psychiatry. 2008; 32: 16771681.

63. Atkuri K, Mantovani JJ, Herzenberg LA, Herzenberg LA. N-acetylcysteine-A safe antidote for cysteine/glutathione deficiency. Curr Opin Pharmacol. 2007; 7: 355-359

64. Tomko RL, Gilmore AK, Gray KM. The role of depressive symptoms in treatment of adolescent cannabis use disorder with N-Acetylcysteine. Addict Behav. 2018; 85: 26-30.

65. Berk M, Copolov DL, Dean O, Lu K, Jeavons S, Schapkaitz I, et al. N-acetylcyteine for depressive symptoms in bipolar disorder-a double blind randomized placebo-controlled trial. Biol Psychiatry. 2008; 64: 468

66. Berger ME, Smesny S, Kim SW, Davey CG, Rice S, Sarnyai Z, et al. Omega-6 to omega-3 polyunsaturated fatty acid ratio and subsequent mood disorders in young people with at-risk mental states: A 7-year longitudinal study. Transl Psychiatry. 2017; 7: e1220.

67. Wozniak J, Faraone SV, Chan J, Tarko L, Hernandez M, Davis J, et al. A randomized clinical trial of high eicosapentaenoic acid omega-3 fatty acids and inositol as monotherapy and in combination in the treatment of pediatric bipolar spectrum disorders: A pilot study. J Clin Psychiatry. 2015; 76: 1548-1555.

68. Trebaticka J, Hradečná Z, Böhmer F, Vaváková M, Waczulíková I, Garaiova I, et al. Emulsified omega-3 fatty-acids modulate the symptoms of depressive disorder in children and adolescents: A pilot study. Child Adolesc Psychiatry Ment Health. 2017; 11: 30.

69. Fristad MA, Young AS, Vesco AT, Nader ES, Healy KZ, Gardner W, et al. A randomized controlled trial of Individual family psychoeducational psychotherapy \& omega-3 fatty acids in youth with subsyndromal bipolar disorder. J Child Adolesc Psychopharmacol. 2015; 25: 764-774. 
70. Fristad MA, Vesc AT, Young A, Fristad MA. Pilot RCT of omega-3 and Individual-Family psychoeducational psychotherapy for children and adolescents with depression. J Clin Child Adolesc Psychol. 2017; 45: 1025-1037.

71. Arnold LE, Young A, Belury MA, Cole RM, Gracious B, Seidenfeld AM, et al. Omega-3 fatty acid plasma levels before and after supplementation: Correlations with mood and clinical outcomes in the omega 3 and therapy studies. J Child Adoles Psychopharmacol. 2017; 27: 223-233.

72. Vesco AT, Young AS, Arnold LE, Fristad MA. Omega-3 supplementation associated with improved parent-rated executive function in youth with mood disorders: Secondary analyses of the omega 3 and therapy (OATS) trials. J Child Psychol Psychiatry. 2018; 59: 628-636.

73. Wallace $C$, Milev R. The effects of probiotics on depressive symptoms in humans: A systematic review. Ann Gen Psychiatry. 2017; 16: 14.

74. Dickerson F, Adamos M, Katsafanas E, Khushalani S, Origoni A, Savage C, et al. Adjunctive probiotic microorganisms to prevent rehospitalization in patients with acute mania: $A$ randomized controlled trial. Bipolar Disord. 2018; 20: 614-621.

75. Messaoudi M, Violl N, Bisso JF, Desor D, Javelot H, Rougeot C. Beneficial psychological effects of a probiotic formulation (Lactobacillus helveticus R0052 and Bifidobacterium longum R0175) in healthy human volunteers. Gut Microbes. 2011; 2: 256-261).

76. Akkasheh G, Kashani-Poor Z, Tajabadi-Ebrahimi M, Jafari P, Akbari H, Taghizadeh M, et al. Clinical and metabolic response to probiotic administration in patients with major depressive disorder: A randomized, double-blind, placebo-controlled trial. Nutrition. 2016; 32: 315-320.

77. Salazar N, Dewulf EM, Neyrinck AM, Bindels LB, Cani PD, Mahillon J, et al. Inulin-type fructans modulater intestinal Bifodobacterium species populations and decrease short-chain fatty acids in obese women. Clin Nutr. 2015; 34: 493-501.

78. Dahiya DK, Puniya M, Shandilya UK, Dhewa T, Kumar N, Kumar S, et al. Gut microbiota modulation and its relationship with obesity using prebiotic fibers and probiotics: A review. Front Microbiol. 2017; 8: 563

79. Kazemi A, Noorbala AA, Azam K, Eskandari MH, Djafarian K. Effect of probiotic and prebiotic vs placebo on psychological outcomes in patients with major depressive disorder: A randomized clinical trial. Clin Nutr. 2018; 38: 522-528.

80. Amirani E, Milajerdi A, Mirzaei H, Jamilian H, Mansournia MA, Hallajzadeh J, et al. The effects of probiotic supplementation on mental health, biomarkers of inflammation and oxidative stress in patients with psychiatric disorders: A systematic review and meta-analysis of randomized controlled trials. Complement Ther Med. 2020; 49: 102361.

81. Luoto R, Kalliomaki M, Laitinen K, Isolauri E. The impact of peri natal probiotic intervention on the development of overweight and obesity: Follow up from birth to 10 years. Int J Obes. 2010; 34: 1531-1537

82. Pärtty A, Kalliomäki $M$, Wacklin $P$, Salminen $S$, Isolauri E. A possible link between early probiotic intervention and the risk of neuropsychiatric disorders later in childhood: A randomized trial. Pediatr Res. 2015; 77: 823-828.

83. Ghaderi A, Asbaghi O, Reiner Z, Kolahdooz F, Amirani E, Mirzaei H, et al. The effects of saffron (Crocus sativus L.) on mental health parameters and C-reactive protein: A meta-analysis of randomized clinical trials. Complement Ther Med. 2020; 48: 102250.

84. Panossian AG. Adaptogens in mental health disorders. Psych Clin North Am. 2013; 36: 49-64.

85. Weng S, Tang J, Wang G, Wang X, Wang H. Comparison of the addition of Siberian ginseng 
(acanthopanax senticosus) versus fluoxetine to lithium for the treatment of bipolar disorder in adolescents: A randomized, double-blind trial. Curr Ther Res Clin Exp. 2007; 68: 280-290.

86. Potter M, Moses A, Wozniak J. Alternative treatments in pediatric bipolar disorder. Child Adolesc Psychiatr Clin N Am. 2009; 18: 483-514.

87. Wurglics M, Westerhoff K, Kaunzinger A, Wilke A, Baumeister A, Dressman J, et al. Comparison of German St. John's wort products according to hyperforin and total hypericin content. Am Pharm Assoc (Wash). 2001; 41: 560-566.

88. Findling RL, McNamara NK, O'Riordan MA, Reed MD, Demeter CA, Branicky LA, et al. An openlabel pilot study of St. John's wort in juvenile depression. J Am Acad Child Adolesc Psychiatry. 2003; 42: 908-914.

89. Simeon J, Nixon MK, Milin R, Jovanovic R, Walker S. Open-label pilot study of St. John's wort in adolescent depression. J Child Adolesc Psychopharmacol. 2005; 15: 293-301.

90. Schaller JL, Thomas J, Bazzan AJ. SAMe use in children and adolescents (letter). Eur Child Adolesc Psychiatry. 2004; 13: 332-334.

91. Kondo DG, Sung YH, Hellem TL, Delmastro KK, Jeong EK, Kim N, et al. Open label uridine for treatment of depressed adolescents with bipolar. J Child Adolesc Psychopharmacol. 2011; 21: 171-175.

92. Kok BE, Coffey KA, Cohn MA, Catalino LI, Vacharkulksemsuk T, Algoe SB, et al. How positive emotions build physical health: Perceived positive communications account for the upward spiral between positive emotions and vagal tone. Psycho Sci. 2013; 24: 1123-1132.

93. Cahn BR, Goodm, Peterson CT, Maturi R, Mills PJ. Yoga, meditation and mind-body health: Increased BDNF, cortisol awakening response, and altered inflammatory marker expression after a 3-month yoga and meditation retreat. Front Hum Neurosci. 2017; 11: 315.

94. Tolahunase MR, Sagar R, Faiqs M, Dada R. Yoga-and meditation-based lifestyle intervention increases neuroplasticity and reduces severity of major depressive disorder: A randomized controlled trial. Restor Neurol Neurosci. 2018; 36: 423-442.

95. Ignácio ZM, Da Silva RS, Plissari ME, Quevedo J, Réus GZ. Physical exercise and neuroinflammation in major depressive disorder. Mol Neurobiol. 2019; 56: 8323-8335.

96. Hughes CW, Barnes S, Barnes C, DeFina LF, Nakonezny P, Emslie GJ. Depressed adolescents treated with exercise (DATE): A pilot randomized controlled trial to test feasibility and establish preliminary effects sizes. Ment Health Phys Act. 2013; 6: 119-131.

97. Merker M, Felder M, Gueissaz L, Bolliger R, Tribolet $P$, Kägi-Braun N, et al. Association of baseline inflammation with effectiveness of nutritional support among patients with diseaserelated malnutrition-a secondary analysis of a randomized controlled clinical trial. JAMA Netw Open. 2020; 3: e200663.

98. Hanaway P. Gastrointestinal dysfunction, chronic disease and the functional medicine matrix model. Presented at the GI Advanced Practice odules. October 25th, 2018. Nashville, TN. 
OBM Integrative and Complementary Medicine 2020; 5(4), doi:10.21926/obm.icm.2004040

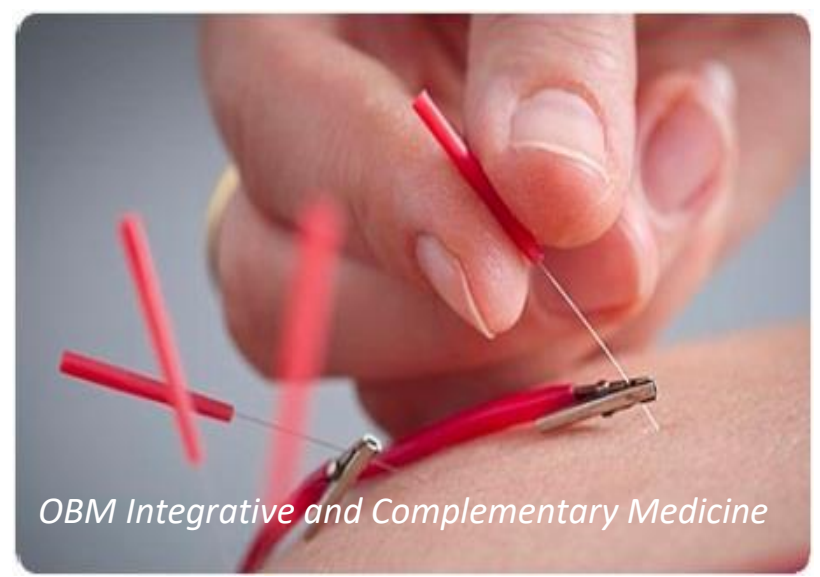

Enjoy $O B M$ Integrative and Complementary Medicine by:

1. Submitting a manuscript

2. Joining in volunteer reviewer bank

3. Joining Editorial Board

4. Guest editing a special issue

For more details, please visit:

http://www.lidsen.com/journals/icm 
Original Research

\title{
Comparing the Perspectives of Professionals and Relatives Toward the Quality of Palliative Care: A Qualitative Study
}

\author{
Emily Reeves ${ }^{1,2,{ }^{*}}$, Brigitte Liebig ${ }^{2,3}$, Eveline Degen Jermann ${ }^{2}$
}

1. University of Basel, Department of Psychology, Missionsstrasse 60/62 4005, Basel, Switzerland; Email: emily.reeves@unibas.ch

2. Fachhochschule Nordwestschweiz FHNW, School of Applied Psychology, Riggenbachstrasse 16, Olten, Switzerland; Emails: brigitte.liebig@fhnw.ch; eveline.degen@fhnw.ch

3. University of Basel, Institute for Sociology, Petersgraben 27, 4051 Basel, Switzerland; Email: brigitte.liebig@unibas.ch

* Correspondence: Emily Reeves; E-Mail: emily.reeves@unibas.ch

Academic Editor: Leila Kozak

Special Issue: Integrative Therapies in Palliative Care

OBM Integrative and Complementary Medicine

2020 , volume 5 , issue 4

doi:10.21926/obm.icm.2004041
Received: July 14, 2020

Accepted: September 23, 2020

Published: October 20, 2020

\begin{abstract}
High-quality care is an important aim of palliative care services. However, the quality of care is a complex phenomenon and is subjectively perceived by those who are closest to the services and are often evaluated poorly. Prior research has highlighted the difficulties in evaluating the quality of care because of different perspectives, and still, only a little is understood about the perception of the quality of care in palliative care services. Investigating the perspectives of key individuals involved in palliative care, such as palliative professionals and relatives of palliative patients, can provide invaluable insight to understand the quality of care. To identify the perceptions of palliative professionals and relatives of the patients toward the quality of palliative care along with identifying the similarities and divergences in the perceptions, as well as highlighting the complexity of quality in palliative care settings while indicating their areas of improvement. This was a qualitative study that involved semi-
\end{abstract}

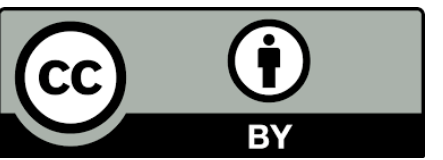

(C) 2020 by the author. This is an open access article distributed under the conditions of the Creative Commons by Attribution License, which permits unrestricted use, distribution, and reproduction in any medium or format, provided the original work is correctly cited. 
structured interviews for the evaluation of palliative care guided by the proposed system approach model [1]. The interviews were conducted with a total number of 38 professionals working in palliative care along with 26 relatives of palliative patients across four Swiss cantons (Ticino, Basel, Vaud, and Lucerne). These professionals worked in primary palliative care (PPC) and specialized palliative care (SPC), where 11 general practitioners (GP's) and 12 nurses were from primary palliative care services. Additionally, 15 interviews were conducted with 9 specialized medical doctors (e.g., oncologists) and 6 specialized palliative care nurses from different hospitals, hospices, mobile palliative care teams (MPCT's), and specialist private practice. The results revealed considerable differences between the perspectives of professionals and relatives regarding the following aspects of care: 1) the availability, 2) clientcenteredness (including respect for patient's wishes and relative's involvement in care, 3) satisfaction (including physical and psychosocial), and 4) communication and information transfer. Mainly, the family members felt that care was not being always readily available and coordinated poorly, the patient's needs were overlooked because of the lack of time. Also, some professionals were perceived to be incompetent with a lack of communication and negligence toward the psychosocial needs of the patient. On the other hand, professionals indicated that relatives may be too involved in the patient care and might have expressed concern due to the high risk of burn out caused by extreme stress and emotionally taxing circumstances of palliative care. The different perspectives uncovered both the similarities and discrepancies, representing a demand for addressing the shortcomings of palliative care services. Specifically, findings indicated that professionals may overestimate the quality of palliative care. Also, future initiatives were needed to ensure that the family members of palliative patients were adequately supported, both psycho-socially and financially, justifying the investment. These findings would help in informing the health policy about the aspects of care that require specific attention to improve the overall quality of care and urge future research to explore the best ways to support these aspects of care.

\section{Keywords}

Palliative home care; quality of care; comparing perspectives; family caregivers; palliative professionals

\section{Introduction}

High-quality care is a vital goal for palliative care services [2], not only because the palliative patients who often have critical demand for effective care services for both pain and symptom management but also to provide good physical and psychosocial support functions [3]. However, quality of care is a complex phenomenon, not necessarily determined by the outcomes of care but constitutes multiple factors and is evaluated subjectively by the individuals closest to care, i.e., the palliative professionals and the relatives of palliative patients [4]. Moreover, ensuring the efficient delivery of care is not always guaranteed and the standard of providing palliative care service is often deemed unsatisfactory [5]. Yet, only little is understood about the perception of the quality of care in palliative services. Thus, the present study aims to explore perceptions of the quality of 
palliative care, as well as highlight the complexity of the phenomenon. Specifically, the objectives are to identify similarities and differences in the perspectives regarding the key elements of the quality of palliative care and also identify the avenues to refine their existing indicators. These are important aims that ensure the clinicians in palliative care settings are well informed about the experiences and perspectives of the patients' relatives, allowing them to potentially adapt and implement the interventions accordingly to serve the patients better and also those who care for them $[4,6]$. Thus, this qualitative study is based on the data concerning the perceptions of quality of palliative care derived from health care professionals working in primary and specialized palliative care settings, as well as the relatives of palliative patients. Their insights are crucial for informing the policy-makers, healthcare managers, and personnel in the sphere of palliative care to identify the potential areas of improvement and promote high-quality of care provision. The following section provides a background to the quality of care specific to the context of palliative care services, including a description of the 'proposed system approach model for evaluating the quality of palliative care' [1], which is the basis of this investigation along with a description about the value of exploring perceptions of palliative professionals and relatives of the patients to understand the quality of palliative care services. Subsequently, the methodology of this qualitative study and its findings are described with the discussion of the findings, followed by the conclusion, recommendations for future research, and limitations of the study.

\subsection{Quality of Palliative Care}

Based on several descriptions in the literature, the World Health Organization (WHO) defines the quality of care as "the extent of health care services provided to individuals and patient population to improve the desired health outcomes" and proposes that to achieve this; the health care must be safe, effective, timely, efficient, equitable, and people-centric [7]. Specifically, the quality of care is considered to be of a high standard if the preferences of patients and relatives are taken into account. Also, their perception of care constitutes the dimensions of quality of care [6]. A highly recognized model for the evaluation of palliative care was developed by Bainbridge and colleagues [1]. According to the 'proposed system approach for the evaluation of palliative care', the following factors are indicative of the quality of care: the availability (i.e., palliative services being accessible to those who need them, in time), the client centeredness (i.e., respect for patient's wishes and adequate involvement of relatives of patients in care), satisfaction (including physical and psychosocial care), and communication and information transfer (i.e., collaboration and transfer of patient information between the palliative care providers). Moreover, for palliative care services to be evaluated positively, it is ought to follow the above-mentioned factors, and only then it can be considered as high standard (i.e., patients and relatives should have frequent, clear communication about the care provided).

\subsection{Perceptions of Quality of Care}

The perspectives of individuals close to palliative patients are imperative for understanding the quality of care in palliative care services [8]. Naturally, the health care professionals (e.g., general practitioners (GP's), nurses, specialized doctors, such as oncologists and specialized nurses), and palliative patients with their relatives, were investigated to understand the quality of care, considering that the extensive experience would make their insights invaluable. However, the 
perspectives of relatives of palliative patients are more relevant, not only because of their closeness to the patient but also because of their increasing involvement in palliative care services [9]. A growing body of research supports that relatives are increasingly being embedded in the palliative care teams, particularly as more aspects of palliative care are provided in the family home environment of the patients [10] and many relatives are receiving palliative training [11] as well to assume the role of primary caregivers, i.e., individuals who provide support without payment [12]. Moreover, while recruiting patients, their critical health status makes it challenging to obtain their feedback for studies to investigate the quality of palliative care [2]. Hence, the insight of those who are closest to the patients is crucial, if not at least a proxy to the perception of patients is needed [13]. Moreover, since palliative care services seek to support not only the patient but also their close relatives [14], their experience of the quality of care also becomes crucial. Yet, research that has compared the perspectives of different people related to care has often identified discrepancies in the perspectives of the quality of care $[8,15,16]$. For example, professionals were shown to overestimate the quality of care delivery to patients and relatives [17]. Identifying the perspectives of quality of care in palliative services allows us to gain a broader, more accurate picture of the quality of care and identify the areas of improvement. However, a majority of the existing literature has investigated perceptions of specific aspects of quality of care, such as perceived patientcenteredness [18] or availability of support services from the relatives [19]. Similarly, in Switzerland, some studies have explored the factors that might indicate the quality of care in palliative settings, such as coordination of care [20] or interprofessional collaboration within the palliative care teams [21]. However, there is a limited understanding of how the quality of care in palliative care services should be perceived. Owing to the gap in research, this article explored the views of the quality of care in palliative services from the perspectives of those who are closest to this field, i.e., palliative professionals and relatives of the patients. Specifically, this study compares their perceptions to bring inconsistencies and similarities to light and to acquire a well-informed understanding of the quality of care in palliative services, according to those closest to the field.

\section{Methods}

The qualitative study design was adopted to explore the perspectives of quality of care from palliative professionals and relatives of palliative patients in Switzerland. Results were based on semi-structured interviews with a total of 38 professionals working in palliative care and 26 relatives of palliative patients. The professionals were from primary palliative care (PPC) and specialized palliative care (SPC), including 11 general practitioners (GP's) and 12 nurses from primary palliative care services, as well as 15 interviews conducted with 9 specialized medical doctors (e.g., oncologists) and 6 specialized palliative care nurses working in different hospitals, hospices, mobile palliative care teams (MPCT's), and specialist private practice. Also, a total of 26 family members of palliative patients participated in 22 interviews and one group interview (involving three daughters and one son of a palliative care patient). The interviews were conducted in different regions/cantons of Switzerland, namely Basel-City, Lucerne, Ticino, and Vaud. These cantons were selected to accurately reflect the rural and urban regions of Switzerland with differently developed policy contexts for palliative care. 
Table 1 Demographic data of the interviewees.

\begin{tabular}{|c|c|c|c|c|c|}
\hline Participants & Number & Gender & & Age (Mean) & Age (Mean) overall \\
\hline & & Female & Male & & \\
\hline GP's (PPC) & 11 & 4 & 8 & 56.6 & \multirow[b]{2}{*}{52.3} \\
\hline Nurses (PPC) & 12 & 12 & 0 & 48.1 & \\
\hline GP's (SPC) & 9 & 8 & 4 & 48 & \multirow[b]{2}{*}{50.6} \\
\hline Nurses (SPC) & 6 & 3 & 1 & 57.7 & \\
\hline Family members & 26 & 19 & 7 & & 60 (59.7) \\
\hline
\end{tabular}

\subsection{Data Collection}

The data for the study were collected between January 2018 and October 2018. In each canton, the participants were identified via a personal referral (i.e., word of mouth) from the professionals who were working in palliative care and were already known to the researchers through prior collaboration, either recruited via email invitation or telephone call. The participants included in the study were interviewed by three researchers in a semi-structured interview format and in a language that corresponded to the official language of the cantons (Vaud: French, Ticino: Italian, or Lucerne/Basel: German). Interviews lasted for $60 \mathrm{~min}$ on an average and were conducted in a location determined by the participant (e.g., clinic, personal office, or the home of the family member of a palliative patient). Interview guidelines were derived from the conceptual framework for the evaluation of integrated palliative care networks [1], which covered the key topics to explore the perceptions of quality of care. Interview questions for professionals included questions about aspects of quality of care, such as satisfaction with care, access to palliative care, and perception about the client-centeredness. Questions about perceptions of satisfaction/access with care included: "Would you say that the services of your palliative care network are available/accessible to all the patients?" and "Are patients satisfied with the care they receive through your network?". On the other hand, perceptions of the client-centeredness included the following questions: "How satisfied are the patients with the communication and information transfer within the framework of palliative care?", "Do you have the impression that family/friends of patients are appropriately involved in the palliative care provided by your network?" and "Do you think that your network can adequately consider the needs of the patients? (medical, non-medical, and spiritual)" (Table 2). While the questions proposed to family members covered a range of questions gauging perceptions about the following aspects of care: the availability/access to care, client-centeredness of care, satisfaction with physical care (including pain and symptom management), and psychological care, as well as the transfer of communication and information. These questions included: "Were the palliative care services (previously) easily accessible to you and your relatives?", "How satisfied is your relative with the physical care that he/she has received?", "How satisfied is your family member with the psychological care that he/she receives?", "Do you feel that your family member is at the center of all decisions, activities, and efforts made by the palliative care team?", "When you think about the wishes, needs, and preferences of your relatives, do you think that they are 
respected by the palliative team?" and "Do you know about your family member's future treatment plan and are you satisfied with the plan?" (Table 3). Participants were encouraged to elaborate on the answers and provide relevant examples from their experience, wherever appropriate. Demographic data was collected from all the participants, and interviews were audio-recorded and transcribed verbatim in their original language, with all the personal identifiers being removed.

Table 2 Interview questions for palliative professionals.

1. Would you say that the services of your palliative care network are available/accessible to all the patients?

2. Are patients satisfied with the care they receive through your network?

3. How satisfied are the patients with the transfer of communication and information within the framework of palliative care?

4. Do you have the impression that family/friends of patients are appropriately involved in palliative care provided by your network?

5. Do you think that your network can adequately consider the needs of patients? (medical, nonmedical, and spiritual)

Table 3 Interview questions for the relatives of palliative patients.

1. Were the palliative care services (previously) easily accessible to you and your relatives?

2. In your opinion, how satisfied is your relative with the physical care that he/she received?

3. In your opinion, how satisfied is your family member with the psychological care that he/she receives?

4. Do you feel that your family member is at the center of all decisions, activities, and efforts of the Palliative Care team?

5. When you think about the wishes, needs, and preferences of your relatives, do you think that they are respected by the Palliative Care team?

6. Do you know about your family member's future treatment plan, and are you satisfied with the plan?

\subsection{Data Analysis}

The data were analyzed using the thematic analysis [22] to identify and report the patterns within. The first stage of the analysis involved familiarization of the data, i.e., listening to the audio 
recordings and reading through the transcripts. The second stage was coding the interview data. Firstly, the data was divided into the transcripts between the interviews of professionals and the ones with the relatives. Two researchers were initially involved in the coding of data, where one researcher coded the data of the interviews with professionals, and the other coded the data of the relatives. The first few transcripts from each dataset were manually coded by the researchers. But, since the transcripts were in different languages, the researchers had to aid one another according to their linguistic ability, where one researcher was fluent in French and German, the other was fluent in Italian. Thus, the researchers explained important segments of the data to one another in their commonly spoken language, English which was otherwise not understandable due to the language barrier. After this, the researchers consulted with one another to compare the discovered perspectives within the data related to the key topics (i.e., availability of care, the client centeredness of care, and satisfaction with care (including physical and psychosocial care), and communication and information transfer). The converging and diverging perspectives between the two interview groups were identified, continuously re-examined, and agreed upon between the researchers to ensure their consensus regarding the codes and analysis. The data in the subsequent transcripts were then coded according to the prominent perspective. Later, the data were coded using a qualitative data analysis software package (MAX QDA) to organize the data efficiently and systematically.

\subsection{Ethical Consideration}

The study received formal research approval from the Ethics Committee of Northwestern Switzerland (EKNZ) on the 29th of August 2018, (Req-2018-00490). Before commencing the interviews, participants received and completed an informed consent form for the study and recording of interviews and the researchers provided them the information about the objectives of the study and the reasons for recording the interviews. Moreover, the concepts of voluntary participation and confidentiality of data were explained to ensure their agreement in the participation of the study.

\section{Results}

The comparison between the perceptions of professionals and the relatives of palliative patients resulted in considerable differences in the following aspects of care: 1) the availability, 2) clientcenteredness (including respect for patient wishes and relatives involvement in care, 3) satisfaction (including physical and psychosocial), and 4) communication and information transfer. These perceptions are compared and described below in detail.

\subsection{Availability of Care}

The availability of care refers to the perception of services being accessible to those who need them, in time [23]. The results indicated considerable divergences in this crucial element of the quality of care, where professionals perceive that they are highly available to patients/relatives to provide palliative services, while relatives perceive that they are: a) poorly informed about the availability of care, b) access to care was a result of independent initiative rather than direction from professionals, and c) despite professionals insisting on the provision of all the relevant information 
about the available palliative services to patients/family members, relatives felt underprepared and also less aware about the palliative care services. These findings are further explained below.

\subsubsection{Perspectives of Professionals}

Professionals did not report problems with the availability of care. Instead, they believed palliative services were easily accessible to patients. According to GPs, patients were well informed about the services available to them. As one of the GPs explained: "they know what services are around and are given all the information they need. We tell them everything there is to know". Another GP supported this notion by adding that plenty of additional information was available for patients online and in hospitals or clinics regarding the care treatments. He quoted: "We have leaflets about services here, and all you have to do is type in "palliative.ch" and offers will come up".

\subsubsection{Perspectives of the Relatives}

In contrast to the professionals, relatives of palliative patients reported the following issues concerning the availability of care. Firstly, they perceive that only limited information about services is provided to them. Additionally, they reported that they must seek the information about services themselves.

3.1.2.1 Limited Provision of Information about Palliative Services. A high number of family members disagree on being informed about the services by professionals rather, they reported that they accessed palliative care services informally, i.e., by hearing about palliative care through friends or family: "It was more through word of mouth, i.e., the people who also had their relatives in palliative care, only told about it and enlightened us (about palliative care)". Moreover, relatives suggest that the degree of information and support provided may be overestimated by GPs, as relatives explained that patients are not always made aware of the services available to them. For example, the daughter of a palliative care patient explains: "They (doctor) said your mother needs something, but then where was it? I do not know whom to approach. Maybe even after a week, nobody gets in touch with me and in the meantime, I do not know where I am supposed to go with her (mother)". Relatives also describe that they were not prepared for palliative care services because it was not explained to them by the professionals. As one mother recalls: "I thought when they found a lump there, she (daughter) would be referred to a gynecologist. I did not know that she needed an oncologist, no one explained that to me. I was suddenly told, "oh, here is the oncologist", which made me realize that it must be cancer".

3.1.2.2 A 'Laissez-faire' Attitude in Providing Palliative Services. Many relatives indicated that the patients and relatives must take an initiative to find out the information about palliative services on their own. For instance, one relative says: "It is accessible and if you type "Palliative Care Luzern", then it comes to this palliative community via Google. I think it is very clear if you are a little interested and look it up yourself", while another respondent remarks: "So, you have to inquire and know where to inquire." Further, the responsibility to organize palliative services for family members are perceived to fall often in the hands of family members and is not facilitated enough by professional support. As one relative explains that the health care professionals did not provide 
enough practical support for accessing palliative care services: "Hospital did not help much. I needed a bed and a wheelchair and I had to organize everything by myself. She (nurse) just gave me the phone numbers." Similarly, another family carer argues that without professional help, it may not be so easy for an average person to find out about palliative care services because one may not be already familiar with the medical services and may struggle to get the information and access to palliative care services: "Because I work at the hospital, it is easier for me and accessible also but I do not know what it is for someone who is not that smart."

3.1.2.3 Limited Awareness of Palliative Care. In a few cases, it was also evident that relatives had limited awareness, not only about the services but palliative care in general. For instance, one family member describes how they were not informed about palliative services and were left to find it out themselves. As one relative describes: "I thought to myself, "But what is palliative care for? What the hell are they doing?" and they just said to me, "make a contact, and you will see". Similarly, a partner of the palliative patient describes that despite being treated in palliative care, her partner was not explained what palliative care services were for and thought that they were only relevant in the case of a terminal illness: "my partner had the feeling that palliative care comes into play only when there is nothing left to do and it comes down to dying". Similarly, another relative explained that she was not made aware of palliative care services until a much later stage of her family member being in care. She did not expect it to be relevant because she had only heard of it in the context of HIV AIDS: "It was only later that the doctor drew my attention toward the possibility of palliative care. Palliative medicine was too little known in my eyes, except in the case of AIDS."

\subsection{Client Centeredness of Care}

The key aspects of client centeredness in care include perceived respect for patient needs and preferences and also the involvement of family members in care, which is discussed below.

\subsubsection{Respect for Patient Wishes}

The results indicated a shared consensus between the professionals and family members of palliative patients about considering and respecting the needs and wishes of the patients during the care process. Notably, many professionals expressed their opinion on the patient being "the center" of all care decisions. As one specialist doctor describes: "Of course, it all depends on the client. We try to take into account all their desires and see them as a person, on the whole". In general, family members agreed that professionals respected the patients' wishes while treating them. One family member quoted an example of their son who was in the care and requested that his father should accompany him to all meetings with the GP that was ensured: "He did not feel confident being in those meetings on his own because it was often overwhelming, but when he asked that I attend and take notes for him, the team did everything to accommodate us. We then worked around a schedule that suited all of us". However, there were also some notable complaints from family members who felt that the patient was not truly at the "center" of care because the health care practitioners working with them were too busy to dedicate time to the patient, hence they did not feel like their family member was a priority. Although one mother acknowledged the inevitability of this situation. Admitting to her frustration, she quoted: "I, of course, accept that the world does not revolve around us and the nurses are busy. But it is not nice to feel that your daughter is just one of 
many patients because they just do not have time to talk to us about what she wants. They have too many other people's needs to think about". Similarly, another relative supported this notion about patients not being given the full attention of health care professionals. Instead, he thought they had to fight for it. Otherwise, they risk being forgotten: "He (the patient) does not draw attention to himself and is very reserved. He does not get attention at all. So, they just leave him alone. Someone who is always shouting gets a lot more attention".

\subsubsection{Relatives' Involvement in Care}

3.2.2.1 Perspectives of the Relatives. A vast majority of relatives reported feeling intensely involved in the care process. Many of them describe playing a crucial role in the care of their loved ones in the treatment plan and decision making. For example, one relative explains the fundamental role in the decision-making process toward the care of the patient: "There was no decision made without her and my consent. We were completely in agreement and involved in the decision-making and reflection process." Similarly, many others also quoted examples of knowing the treatment plan and schedule of their relatives' care to support their involvement and often described themselves as a part of the care team. As one daughter explains: "I have a black file here filled with every detail about when and which medications she should take. I know everything at this point. The nurses leave me to tend to her bandages and so on because they know I am no different to them, I am also one of her carers". However, in two cases, the family members reported that they did not feel involved enough in the care process, not because the professionals failed to keep them involved, but because of the resistance from the patient. As explained by a mother: "He (son) refuses to let me in the meetings. He wants his treatment to be between him and his doctors, and I cannot override that, unfortunately, I am just on the sidelines".

3.2.2.2 Perspectives of the Professionals. Most professionals agree that family members are fully involved in the care. As described by one nurse: "I know mothers and daughters who could tell you more about the pain medications of their relative than the nurses on the same team who are there for years. They are absolutely involved!". However, in some cases, professionals felt that relatives were too involved. For instance, one GP feared that the relative risked 'burn out' from the extent of his involvement with care: "I saw the husband doing everything he could, he invested everything at the cost of his career, his training. It was incredible and there was little compensation. There was no tax allowance to support this. I have to be very careful about the burn-out of the spouse who could not take it anymore. The spouse can sometimes get out of control, and then psychologically, it becomes difficult.". This is in line with a specialist doctor who admitted to not include the family members much because they are too emotionally invested. The specialist feels wary of involving relatives too intensely in the decision-making process: "When it comes to making decisions, unfortunately, they cannot be trusted to make the 'best' decisions, let's say because their emotions are in it too". However, despite family members feeling extremely involved in the care process, responses from some GPs suggest that the family members may not be as involved as they might think. Another doctor admits that he with-holds the information from relatives in some cases: "I do not want to gloss over this, of course, conversations also take place behind the patient's back, or it is expressed that one should not communicate certain things with the patient, which is quite difficult for me, it is legally a gray area". Similarly, a few GPs mentioned difficulties in involving the families for practical reasons such as not all family members agree on a course of action related to the care. 
As one doctor describes: "Families are always involved but it is not always easy with them, because you have a son from Geneva who does not agree with the son from Lausanne. The son from Lausanne thinks that you should not put her (mother) in a palliative care center and she should die at home while the one from Geneva thinks it is better to have care."

\subsection{Satisfaction with Care}

Satisfaction with care describes the perception of how satisfied professionals and family members think the patients to be with their overall care, including physical and psychosocial care. The perspectives are further described below.

\subsubsection{Perspectives of the Professionals}

The results demonstrated that both the professionals and family members perceived the patients to be satisfied with the care. However, professionals often acknowledged their limited capacity in estimating the satisfaction of patients. When asked by a family doctor whether he believed his patients to be satisfied with the care they received, he said: "I do not know. How do we evaluate that? (Should we ask) Was it all right? How are you doing? Most of the people I sent to a mobile unit or hospital felt satisfied. But it is only an impression". Yet, on the whole, professionals generally believe that patients were satisfied. One nurse even goes as far as to say: "I do not think I have ever known a patient to be truly unhappy with the care they get here".

\subsubsection{Perspectives of the Relatives}

Although the majority of family members seem to be happy with the care and feel that their family members were content with the received palliative care, many family members also raised some specific issues negatively impacting their perception about satisfaction with care. Notably, relatives often attributed the dissatisfaction with overall care to a) poor coordination, b) a perceived lack of professional competency, and c) poor continuity of care. Moreover, family members also had complaints about physical and psychosocial care, especially a lack of psychosocial support. The aspects of care to perceived satisfaction are expanded below.

3.3.2.1 Poor Coordination of Care. The relatives commonly expressed that palliative care teams were poorly coordinated. For instance, a relative describes that her mother's care was compromised due to the poor coordination of care and time: "It was badly organized. The nurses never came on time. They also had no plans about when to come. It was agreed at about 8 o'clock, but by the time they came, it would be 10 o'clock. In the meantime, my mother was lying on the bed with wet diapers and could not cope anymore". Similarly, one person explains a situation where the care was badly coordinated because professionals were not sure of the medications being already received by the patient: "They were not well informed and did not appear to know what was going on. They did not even know if they would give him any medication. When we saw that they were not sure, I was not happy". Additionally, some relatives mentioned their unhappiness with the care because the coordination was unreliable. One relative describes their frustration: "Depending on the time, there were very quick changes happening, and every time there was someone else. So, consistency was often missing, actually continuously (missing)". Similarly, another relative pointed out that they could not answer the question about how satisfied they were with the care because of the 
unpredictability of the level of care: "One day you might get the best team ever and it is all great, but just as quickly, you find yourself with strangers on the team, and it is just the opposite of great!"

3.3.2.2 Poor Continuity of Care. Continuity of care is an important aspect that refers to the relational continuity (the patient is continued with the usual practitioner), informational continuity (continued communication and knowledge for easy flow of patient information between the involved care providers), and management continuity (coordination for transitioning between care providers to be clear and seamless for the patient) [24]. Some family members describe their frustration: "I cannot do anything for you anymore (said by a professional). That is another shock you can imagine". Understandably, family members also reported suffering because of the sudden drop in communication with professionals after they no longer needed their care or were transferred to another care facility. As one relative describes, there was no form of continuity of care once the treatment for the patient ended: "From one day to the next, I went from seeing their (nurses) faces any time I needed them, to suddenly being all alone again. I think that is a terrible part of care because my life goes on, but the care just stops". Yet another person explains a situation where there was no continuity of care after the death of their partner and they were left without knowledge about the next steps: "When my partner died, the only thing I thought was, now I have to call somebody, and I do not know who, I do not know what to do now."

3.3.2.3 Lack of Professional Competency. Alarmingly, quite a few family members reported dissatisfaction with the quality of care because they felt that the professionals were incompetent. As one person puts it bluntly: "I was not happy with the home care service because they may be nice people but they are not competent". Similarly, another relative was unhappy with the care and wanted to replace the professional because she felt that the professional was not competent: "Let's just say, I had to deal with a nurse who was not up to the task. I talked to the head nurse about it and said, "This one (nurse) has to get out of here. It is not okay at all". Another relative speculates that the standard of care could be lower now because of the lack of sufficient training: "The level of care has gone down. I have the impression that it has dropped because some foreign doctors do not have the level of Swiss studies (education)". In another situation, the relative implies that the competency was so poor that they liked to step in themselves to carry out the treatments: "I liked to be a volunteer for a while because I noticed that some treatments were sometimes poorly done". She goes on to explain that the nurses failed to meet the needs of the patient and were not attended in times of need: "For example, they did not give her anything to drink for a very long time and she was dying of thirst after she was cold, and then she was hot because of her changing temperature but no one passed in to help."

\subsubsection{Physical and Psychosocial Care}

Physical care involves medical and practical aspects along with pain and symptom management [25] while the psychosocial care involves meeting the emotional, psychological, and existential needs of palliative patients and their families, and help in alleviating grief, fear, and other psychological and social problems [1]. The results indicated the following key perspectives: a) psychosocial care was lacking, b) physical care was prioritized over psychosocial care, and c) relatives felt conflicted about the approaches to pain and symptom management. The data on these perspectives are discussed below. 
3.3.3.1 Lack in Psychosocial Care. Professionals support that many services are available for treating both physical and psychological needs, and doctors and nurses do attend to these demands of patients and their relatives. As one adamantly puts: "It is not just a job, but it is my duty to make sure that they (patients) are looked after in every way imaginable. This is palliative care; it is about giving them the best possible life at this stage". Despite this, both family members and professionals seemed to agree that psychosocial care is considerably lacking compared to physical care. One GP expresses that they are not comfortable providing psychological support compared to the physical support and admit to not being concerned with the 'spiritual' needs: "Every day, medical needs I can manage, but not spiritual needs. We are not very good at this. I am rarely interested in knowing what the patient's spiritual position is. I am rather involved in all kinds of things about the pain but I am not very good at this psychological thing". Moreover, another GP implies that although psychological issues should be addressed, it is difficult to provide the support because these problems are expected and are considered 'normal' in palliative care: "Spiritual needs should not be neglected, nor depression, but when you realize, "She has cancer", it is normal that she is sad". What should we do about her depression?" This may account for why professionals also report that they do not necessarily advertise the options for psychosocial support but rather only provide it when requested. As one GP explains: "There are psychologists and support groups available, especially for bereaved widows but I give this information only when asked. I do not usually ask them (patients) about their spiritual needs".

3.3.3.2 Physical Care Prioritized Over Psychosocial Care. Perhaps, such thinking from the professionals might account for many family members also reporting their relatives' psychological needs not being taken as seriously as the physical ones. One concerning example was provided by a relative who describes that their relative received proper care only when she started threatening to take her own life: "She wanted to get it over with and she said, "I am going to get a knife and kill myself" and then I went to the doctor and said, "Now you listen to what she said to me and do something" and then they said, "OK, we heard her despair". Oh, yes only then it was more physical, that is what they meant by 'despair'. We had to wait a long time because I felt the despair long before that". Also, family members admit having limited knowledge about where to receive the psychological help, as one father describes: "I have heard of churches sending people out to come and talk with you. But I am not sure that this is something people like us would have the chance to do".

3.3.3.3 Relatives Feeling Conflicted about Pain and Symptom Management. On the other hand, some family members reported feeling conflicted about physical care, including pain and symptom management (i.e., commonly, the painkillers or sedatives, e.g., morphine). Although the majority of relatives desired their family members in care to be in as little pain as possible, they also struggled with the perceived negative impact on the patients' quality of life. As one relative describes the adverse effects of pain killers: "Well, we try to relieve the symptoms, and calm the pain, but the more we subside the pain, the more the person's personality goes away, which I find difficult to bear. She (family member) is concerned that she would "end up like a vegetable" and that decision would be made in her place." Another mother describes a similar concern: "If we say, yes, up the dosage, then the doctor will do so, but at what cost? Perhaps her life will be spared, but what kind of life are you saving? She is totally out of it, on medications". 


\subsection{Satisfaction with Care}

Communication and information transfer refer to all the aspects of the provider's collaboration and patient-centered interactions [1]. The sharing of information that is relevant, accurate, transparent, concise, and timely is an essential element to ensure a high quality of palliative care [26]. Responses from professionals and relatives echoed similar concerns for communication between the relatives, patients, and professionals and the transfer of important care information. Crucially, the majority of professionals reported issues with finding time to talk with patients or their relatives. As one assistant nurse explains: "It goes without saying that we are all busy around here. I do my best, but I am limited to quick pop-ins". Another GP admitted that despite trying to reassure the patients that he is available for them, he often misses the phone calls and cannot attend meetings: "I tell them I am reachable $24 / 7$ which is technically true but practically not. My days are so full, one visit runs over and then I cannot make the next one". Also, some family members reported that information about patient care was poorly explained or lacking, as one relative describes: "They (nurses) explained it badly to us. They should have said when she is unconscious or she sleeps then you should not give her anything to drink, but they did not tell us that." In other cases, relatives describe miscommunications because the information was not passed on to them directly. One family member describes that they waited a long time for nothing because a message was relayed to them: "She (nurse) should have come but had an emergency and could not come. She reported it to the central office, but then they forgot to pass on the message to us and we waited two hours after all that."

\section{Discussion}

The findings of this study highlighted important aspects of the quality of palliative care. Specifically, in indicating the valuable perspectives and experiences of the professionals and relatives of palliative patients regarding the availability, client centeredness, satisfaction with care, and communication and information transfer.

Firstly, the data reveals a high level of disagreement between professionals and relatives' opinions toward the availability of care. Crucially, although professionals perceived that palliative services were highly available to patients and their relatives, relatives expressed being poorly informed about it. Instead, they felt 'left alone' to discover palliative services for themselves, or when encouraged to seek palliative services, the responsibility to take the initiative was on them. It was also apparent that professionals mainly understood care to be available to patients because of the presence of infrastructure for services. However, relatives described that since professionals did not inform patients about these services, it was not easily available or accessible. The experiences of relatives describing the situations where professionals do not adequately inform patients about the availability of care are highly concerning because failing to provide patients with care information could result in missing out on the appropriate treatment, which at the palliative level may contribute to further deteriorating health outcomes to already fragile patients. A vast body of literature supports that misinformation can also negatively impact the quality of patient care because the coordination of care is prohibited by inaccurate or untimely information [27]. If the patients who require specialized care in palliative services are not made aware of or connected to these services, then they may risk neglecting their health at a potentially critical point of their illness trajectory [1]. If this is the case, as the relatives imply, where patients and relatives are not 
sufficiently informed about the services available to them and/or they must rely on themselves to reach out to services, then it not only indicates a considerable failing on behalf of professionals to meet the patient needs but also clearly demands them to re-evaluate their approaches in providing the service information.

Another important finding is the divergent perceptions of the client centeredness, especially the involvement of relatives in care. Adequate involvement of relatives in care is a vital aspect of the quality of care [9]. Yet, the responses indicated that while the relatives and professionals agreed on relatives being highly involved in care, they differed in the opinions for appropriateness of their involvement. Specifically, health care professionals often expressed concern with the extent of involvement of relatives in the process of care. Many explained that they were worried about the family members taking too much responsibility for their relatives' care and risked psychological distress, including 'burn-out'. Consequently, a few GPs alluded to concealing information from the family members regarding the patient, out of the fear of causing too much stress, particularly during decision-making. The concerns expressed by the GPs here are legitimate, especially because extensive literature supports the association between the caregivers and a high risk for a whole host of adverse psychological effects, including, but not limited to depression, anxiety, burn- out, stress, fatigue, and insomnia $[28,29]$. The conflict expressed by the GPs here needs the relatives to be adequately involved in care but also raises the question about the extent of their involvement in care, or, in what capacity? There is a growing body of evidence to support that relatives are not only involved in care but are also becoming an increasingly fundamental part of the palliative care team [30]. In many cases, family members are described as 'primary caregivers' in palliative care literature, i.e., individuals who provide most of the care to the patients without payment [12]. While other countries, including the United Kingdom and the United States, have adopted strategies to financially support such primary caregivers [31], Switzerland does not yet support the health regulations enabling such initiatives to a large extent. Relatives living in the same household may receive some financial reimbursement, but that does not apply to all cases, especially those who care for the patients living separately from them [32]. This may also contribute to the burden that health care professionals have described in the data. Adequate reimbursement in health care is of utmost importance to ensure good quality care and alleviate stress for carers [33]. Beyond that, appropriate training and emotional support should be provided to equip the relatives to manage the high level of involvement while taking care of their loved ones.

The concerns raised by the relatives of palliative patients related to the satisfaction of care are important discoveries made in this study because health care professionals in the field often felt that they could not necessarily comment on the satisfaction of patients. The relatives were able to shed light on the specific issues that negatively impacted their evaluation of care, they also pointed out several factors, such as poor coordination, poor continuity, as well as negligence of psychosocial needs, and a perceived lack of professional competency. Poor care coordination is consistently identified as a problematic facet of care in the context of palliative services [34]. These results echo recent literature concerning the coordination of palliative care services in Switzerland highlighting some of the problems described here, such as the poor organization of care resulting from frequent changes happening in the care team, miscommunication, and a lack of time for care coordination [17]. They further advocated the need for improvement in this regard. Regarding the perceived poor continuity of care, in context to the problems with communication and information transfer, findings indicated a need for strengthened communication between the patients, relatives, and 
providers, and one way of aligning this is through the allocated care coordinators who facilitate the care coordination and communication between the members of the palliative team [20]. Notably, palliative care settings with methods such as the employment of case managers who manage the smooth transitioning of patient care continuity have been well received by the patients and relatives [35].

\section{Conclusion}

This study brought different perspectives toward the quality of palliative care to light. Significantly, professionals working in the field and relatives of palliative patients held different beliefs toward the key aspects of care, including the availability and access to care, client centeredness of care, satisfaction with care (especially in case of psychosocial care), and communication and information transfer. Although there was a consensus between the professionals and family members for various dimensions of care, this study highlighted important apparent divergences between professionals and relatives' perspectives. Significantly, the interviews of the family members showed that though the health care professionals strive to provide the highest quality of care, there are some aspects where it lacks. The primary concerns were that care was not always readily available, poorly coordinated, patient needs were overlooked because of the lack of time, few professionals were perceived to be incompetent, communication was lacking, and psychosocial needs were neglected. Professionals agreed that psychosocial needs are not well addressed as the physical needs and also reported communication problems. However, professionals also raised other concerns with aspects of care, where they felt that though it is good for relatives to be involved in care, their over-involvement may lead to a high risk of burn out caused by the high stress and emotionally taxing circumstances of palliative care. These different perspectives, including both similarities and discrepancies, represent a demand for addressing the shortcomings of palliative care services.

\section{Recommendations}

In response to the findings of the study, the following propositions are suggested for the health policies of palliative care in Switzerland. Firstly, because of the significant involvement of family members of palliative patients in the process of care, adequate psychosocial and financial support should not only be available but mandatorily provided in the case of family members being primary caregivers. This measure is proposed to alleviate the psychological and financial burden from relatives and reduce the risk of adverse health and financial outcomes often caused by being involved in palliative care. Additionally, professionals in the field are urged to provide relevant information about the availability of basic palliative care along with psychosocial support. The education of palliative professionals should ensure that they are equally equipped to cater to the psychological issues as they are to physical ones to avoid the negligence of psychosocial needs. For the coordination issues faced in palliative care teams, designated care coordinators should be employed to mitigate the miscommunication and untimely delivery of information. 


\section{Limitations}

This study relies on the perspectives of professionals and family members. However, it would be invaluable to have palliative patients' insight and perceptions for understanding the patients' experience of the quality of care in palliative services. This information could serve important knowledge to inform the policy and provide recommendations concerning the quality of care in palliative services with patient wishes in mind. Another drawback of the study, as acknowledged by some of the interviewed health care professionals, is that it is difficult to accurately assess the quality of care without having measurable scales, and it is uncertain to what extent professionals and relatives can evaluate the standard of care since they are not the patient themselves. Specifically, many studies support that health care professionals tend to overestimate the patients' satisfaction and overall quality of care provision [36]. Although it is recognized that health care professionals and relatives of patients may have an excellent insight into the patients' experience of care, the most accurate understanding of the quality of a patient's care would inevitably be the one directly coming from the patient.

\section{Acknowledgments}

The authors wish to thank all the participants of the study for kindly contributing their expert knowledge and insights of palliative care, which were crucial to explore the quality of palliative care. The authors also wish to thank the Swiss National Science Foundation (SNF), NRP 74 "Smarter Health Care" (www.nrp74.ch; Schweizerischer Nationalfonds zur Förderung der Wissenschaftlichen Forschung) for funding this research.

\section{Author Contributions}

Ms. Emily Reeves is the corresponding author of this work. Ms. Emily Reeves and Dr. Prof. Brigitte Liebig were responsible for the conceptual development of this work. Additionally, Ms. Emily Reeves carried out the write up of this research, whilst Dr. Prof. Brigitte Liebig expertly edited and refined the paper. Ms. Emily Reeves and Eveline Degen-Jermann carried out the qualitative interview studies and were responsible for the qualitative analysis of the interview data. The share of labour with respect to the analysis of the interviews was divided equally between Ms. Eveline Degen and Ms. Emily Reeves.

\section{Competing Interests}

The authors have declared that no competing interests exist.

\section{References}

1. Bainbridge D, Brazil K, Krueger P, Ploeg J, Taniguchi A. A proposed systems approach to the evaluation of integrated palliative care. BMC Palliat Care. 2010; 9: 8.

2. Hanson LC, Bull J, Wessell K, Massie L, Bennett RE, Kutner JS, et al. Strategies to support recruitment of patients with life-limiting illness for research: The palliative care research cooperative group. J Pain Symptom Manag. 2014; 48: 1021-1030. 
3. Darzi A. High quality care for all: NHS next stage review final report. London: Department of health; 2008. Available from: https://www.gov.uk/government/publications/high-quality-carefor-all-nhs-next-stage-review-final-report.

4. High quality-safe services-quality and patient safety in the health and care services. Report to the storting (norwegian parliament) No. 10. Olso: Ministry of Health and Care Services; 20122013. Available from: https://www.regjeringen.no/no/dokumenter/meld-st-1020122013/id709025.

5. Wittenberg-Lyles E, Parker OD, Demiris G, Baldwin P, Regehr K. Communication dynamics in hospice teams: Understanding the role of the chaplain in interdisciplinary team collaboration. J Palliat Med. 2009; 11: 1330-1335.

6. Bengoa R, Kawar R, Key P, Leatherman S, Massoud R, Sturno P. Quality of care: A process for making strategic choices in health systems. Geneva: World Health Organization (WHO); 2006. Available from: http://www.who.int/iris/handle/10665/43470.

7. What is quality of care and why is it important? Geneva: World Health Organization (WHO); 2020. Available from: https://www.who.int/maternal child adolescent/topics/quality-ofcare/definition/en.

8. Austin L, Luker K, Caress A, Hallett C. Palliative care: Community nurses' perceptions of quality. Qual Health Care. 2000; 9: 151-158.

9. Burns CM, Abernethy AP, Grande DE, David CC. Uncovering an invisible network of direct caregivers at the end of life: A population study. Palliat Med. 2013; 27: 608-615.

10. Gomes B, Calanzani N, Higginson I. Reversal of the British trends in place of death: Time series analysis 2004-2010. Palliat Med. 2012; 26: 102-107.

11. Hauser JM, Kramer BJ. Family caregivers in palliative care. Clin Geriatr Med. 2004; 20: 671-688.

12. Kitrungrote L, Cohen MZ. Quality of life of family caregivers of patients with cancer: A literature review. Oncol Nurs Forum. 2006; 33: 625-632.

13. Aasb $\varnothing$ G, Rugkåsa J, Solbraeække KN, Werner A. Negotiating the care-giving role: Family members' experience during critical exacerbation of COPD in Norway. Health Soc Care Community. 2007; 25: 612-620.

14. Brobäck G, Berterö C. How next of kin experience palliative care of relatives at home. Eur J Cancer Care. 2003; 12: 339-346.

15. Oosterveld-Vlug MG, Custers B, Hofstede J, Donker GA, Rijken PM, Korevaar JC, et al. What are essential elements of high-quality palliative care at home? An interview study among patients and relatives faced with advanced cancer. BMC Palliat Care. 2019; 18: 96.

16. Ke YX, Hu SH, Takemura N, Lin CC. Perceived quality of palliative care in intensive care units among doctors and nurses in Taiwan. Int J Qual Health Care. 2019; 31: 741-747.

17. Vedel I, Ghadi V, Lapointe L, Routelous C, Aegerter P, Guirimand F. Patients', family caregivers', and professionals' perspectives on quality of palliative care: A qualitative study. Palliat Med. 2006; 28: 1128-1138.

18. Brazil K, Bainbridge D, Ploeg J, Krueger P, Taniguchi A, Marshall D. Family caregiver views on patient-centred care at the end of life. Scand J Caring Sci. 2011; 26: 513-518.

19. Hudson P, Payne S. Family Caregivers and palliative care: Current status and agenda for the future. J Palliat Med. 2012; 14: 864-869. 
20. Reeves $E$, Schweighoffer R, Liebig B. An investigation of the challenges to coordination at the interface of primary and specialized palliative care services in Switzerland: A qualitative interview study. J Interprof Care. 2020; 26: 1-7.

21. Alvarado V, Liebig B. Inter-professional collaboration between family doctors and nurses at the end of life. Challenges of community-based palliative care in Switzerland. J Comm Pub Health. 2016; 2: 124.

22. Braun V, Clarke V. Using thematic analysis in psychology. Qual Res Psychol. 2006; 3: 77-101.

23. Shaller D, Consulting S. Patient-centered care: What does it take? New York: The commonwealth fund; $2007 . \quad$ Available from: https://www.researchgate.net/publication/228787332 Patient Centered Care What Does It Take.

24. Reid Rj, Haggerty J, Mckendry. Defusing the confusion: Concepts and measures of continuity of health care. Final Report. Ottawa: Canadian Health Service Research Foundation; 2002. Available

from: https://www.researchgate.net/publication/245856177 Defusing the Confusion Concepts a nd Measures of Continuity of Health Care.

25. Ferris FD, Von Gunten CF, Emanuel LL. Ensuring competency in end-of-life care: Controlling symptoms. BMC Palliat Care. 2002; 1: 5.

26. Oandasan I, Baker GR, Barker K, Bosco D, D'amour D, Jones S, et al. Teamwork in healthcare: Promoting effective teamwork in healthcare in Canada Ottawa. Ottawa: Canadian Health Services Research Foundation; 2006. Available from: https://www.researchgate.net/publication/249940003 Teamwork in Healthcare Promoting Effective Teamwork in Healthcare in Canada.

27. Luckett T, Phillips J, Agar M, Virdun C, Green A, Davidson P. Elements of effective palliative care models: A rapid review. BMC Health Serv Res. 2014; 14: 136.

28. Sand L, Strang P, Milberg A. Dying cancer patients experiences of powerlessness and helplessness. Support Care Cancer. 2008; 16: 853-862.

29. Totman J, Pistrang N, Smith S, Hennessey S, Martin J. You only have one chance to get it right: A qualitative study of relatives experiences of caring at home for a family member with terminal cancer. Palliat Med. 2015; 29: 496-507.

30. Woodman C, Baillie J, Sivell S. The preferences and perspectives of family caregivers towards place of care for their relatives at the end-of-life. A systematic review and thematic synthesis of the qualitative evidence. BMJ Support Palliat Care. 2016; 6: 418-429.

31. Gardiner C. Exploring the financial impact of caring for family members receiving palliative and end-of-life care: A systematic review of the literature. Palliat Med. 2014; 28: 375-390.

32. Nationale strategie (National strategy) palliative care 2013-2015. Bern: Federal Office of Public Health (FOPH); 2012. Available from: https://www.pallnetz.ch/cm document/07 D Nationale Strategie Palliative Care 20132015.pdf.

33. Travis S, Hunt P. Supportive and palliative care networks: A new model for integrated care. Int J Palliat Nurs. 2001; 7: 501-504.

34. Reeves S, Xyrichis A, Zwarenstein M. Teamwork, collaboration, coordination, and networking: Why we need to distinguish between different types of interprofessional practice. J Interprof Care. 2018; 32: 1-3. 
35. Ozcelik H, Fadiloglu C, Karabulut B, Uya M. Examining the effect of the case management model on patient results in the palliative care of patients with cancer. Am J Hosp Palliat Med. 2014; 31: 655-664.

36. Hoffmann TC, Del Mar C. Clinicians' expectations of the benefits and harms of treatments, screening, and tests: A systematic review. JAMA Intern Med. 2017; 177: 407-419.

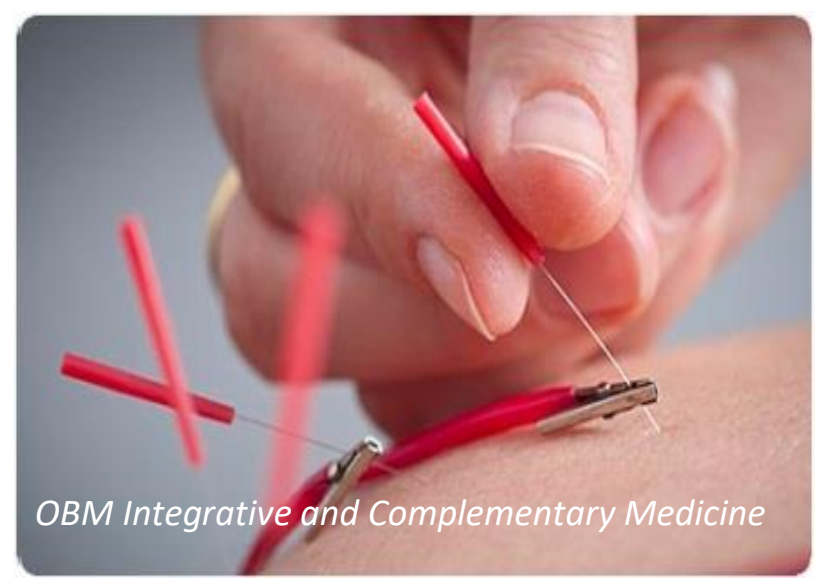

Enjoy OBM Integrative and Complementary Medicine by:

1. Submitting a manuscript

2. Joining in volunteer reviewer bank

3. Joining Editorial Board

4. Guest editing a special issue

For more details, please visit: http://www.lidsen.com/journals/icm 
Opinion

\title{
A Potential Role of Coenzyme Q10 Deficiency in Severe SARS-CoV2 Infection
}

Vasilios M. Polymeropoulos*

2200 Pennsylvania Avenue NW Suite 300E, Washington, DC, USA; E-Mail: vasilios.polymeropoulos@vandapharma.com

* Correspondence: Vasilios M. Polymeropoulos; E-Mail: vasilios.polymeropoulos@vandapharma.com

Academic Editor: Sok Cheon Pak and Soo Liang Ooi

Special Issue: Complementary, Traditional, and Integrative Medicine for COVID-19

OBM Integrative and Complementary Medicine 2020, volume 5 , issue 4

doi:10.21926/obm.icm.2004042
Received: August 01, 2020

Accepted: October 16, 2020

Published: October 26, 2020

\begin{abstract}
There is a dramatic need for extensive research into the predictors of severe infection with SARS-CoV2 and therapeutic options for infected people. People who suffer from severe illness and higher mortality display a pattern of having specific co-morbidities (diabetes, obesity, hypertension) and are of higher age. Recent research has described methods of viral entry via receptors (ACE2, TMPRSS2) and the hyper-inflammatory state often associated with severe illness (increase in interleukins, increase in TNF-alpha). These discoveries have led to the research of currently available and developing therapies, that are helpful to patients. Deficiencies of specific vitamins and other endogenous molecules of the body should be examined to understand if a pattern exists among the people most severely affected. Coenzyme Q10 (CoQ10) is a fat-soluble substance ubiquitously expressed throughout the body that is important for the generation of ATP and mediation of inflammatory disease. CoQ10 faces a decline with increasing age, genetic predispositions, and ingestion of exogenous compounds that could reduce the level of CoQ10. Deficiencies and subsequent
\end{abstract}

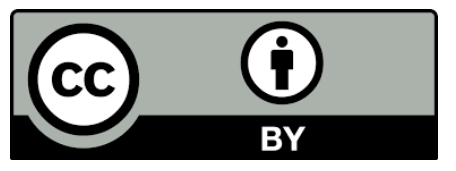

(C) 2020 by the author. This is an open access article distributed under the conditions of the Creative Commons by Attribution License, which permits unrestricted use, distribution, and reproduction in any medium or format, provided the original work is correctly cited. 
supplementation with CoQ10 recently has displayed encouraging results for the improvement of a wide variety of diseases. This manuscript is significant as it points to a potential relationship of CoQ10 and the population suffering from severe illness of COVID-19, and further encourages the need for research into measuring the levels of CoQ10 and vitamins to understand if levels predict severe illness and mortality. This could offer new avenues into research in combating this pandemic and potentially future therapeutic options.

\section{Keywords}

COVID-19; Coenzyme Q10; antioxidant; SARS-CoV-2; inflammation

\section{Introduction}

Oxidative stress plays an important role in viral infection through multiple mechanisms, including the reduction of the host antioxidant response [1, 2]. The global scientific community is rapidly trying to delineate the pathophysiology of disease with SARS-CoV2 infection, by identifying associated biomarkers of severe illness in order to discover potential therapeutics. An examination of associations between levels of critical antioxidants such as Coenzyme Q10 (CoQ10), and severity of SARS-CoV2 infection should be examined, as potential associations may indicate markers of disease severity and possibly may have a causative role.

CoQ10 is a fat-soluble molecule that is a member of the ubiquinone family [3]. CoQ10 is ubiquitous in humans and present in most cells, and is both synthesized endogenously and acquired exogenously [3]. The highest levels of CoQ10 are in organs with the highest metabolic demand such as the heart, lung, kidney and liver [3]. CoQ10 has several important physiological roles including acting as an essential cofactor in the electron-transport chain to generate ATP and serving as a lipid antioxidant, neutralizing free radicals thereby preventing ensuing damage to the body [3]. Other researchers have also suggested a deficiency in CoQ10 allows for increased cellular damage from oxidative stress, potentially propagating the cytokine storm hypothesized to be driving severe illness associated with SARS-CoV2 infection [4]. A combination of reactive oxygen species (ROS) and viroporins due to viral infection, may be leading to the stimulation of pro-inflammatory signals, causing the cascade of inflammatory disease in susceptible individuals with COVID-19 pneumonia [5].

Levels of CoQ10 can be diminished for several reasons including advanced age, the effects of compounds interfering with synthesis, and genetic factors. Statins are a major class of compounds highly associated with diminished CoQ10 levels. Statins inhibit HMG-CoA reductase reducing synthesis of cholesterol and levels of CoQ10 due to the inhibition of a common pathway of synthesis [6]. Atorvastatin was found to decrease the level of CoQ10 by $49 \%$ within 14 days of treatment [6]. Supplementation with CoQ10 has demonstrated benefit in a variety of diseases. MELAS syndrome, a genetic disease leading to impaired mitochondrial energy production and multi-organ disease, has been treated with CoQ10 [7]. In young women with decreased ovarian reserve, where increased oxidative stress and lower levels of CoQ10 were found to be associated with disease, CoQ10 supplementation significantly improved the number of retrieved oocytes and fertilization rate [8]. CoQ10 may also have benefit in reducing cardiovascular fibrosis associated with aging [9]. CoQ10 
levels peak around age 20 , followed by an age-dependent decrease over time [10]. The largest tissue specific decrease at age 80 occurs in the lungs (51.7\% from peak) and heart (42.9\% from peak) [10]. Mutations of several genes (primarily the COQ genes) involved in CoQ10 biosynthesis can result in a deficiency [11].

\section{CoQ10 Anti-Inflammatory and Anti-Oxidant Roles in Disease}

CoQ10 has an integral anti-inflammatory role in the body as a free radical scavenger, and has been explored in the treatment of a variety of inflammatory mediated diseases.

Treatment with CoQ10 has been evaluated in several diseases causing critical illness. CoQ10 supplementation improved survival and decreased pulmonary edema in sepsis-induced acute lung injury in rats [12]. Relatedly, in patients with septic shock, CoQ10 levels were found to be lower and correlated with higher levels of inflammatory markers [13].

CoQ10 supplementation has also been evaluated in several inflammatory disease models of platelet aggregation, fibrosis, and chronic inflammatory disease. Inhibition of platelet aggregation by CoQ10 was found to occur through multiple pathways, including the up-regulation of cAMP and PKA, and through the inhibition of vitronectin (CD51/CD61) [14, 15]. CoQ10 supplementation has been found to be beneficial in attenuating fibrosis in the lung and liver in rats through up-regulation of autophagy processes [16]. Supplementation with CoQ10 improves liver and systemic markers of inflammation in people with nonalcoholic fatty liver disease [17].

The utility of supplementation with CoQ10 has also been examined in cardiac and vascular disease. CoQ10 supplementation improves mortality and cardiac markers in people with heart failure $[18,19]$. Total cholesterol and low-density lipoprotein levels improve in people with diabetes with CoQ10 supplementation [20]. Supplementation with CoQ10 has been found to improve endothelial dysfunction in people with dyslipidemia [21].

Regarding a potential role in viral infection, CoQ10 has been shown to be lower in patients with acute influenza [22]. A study of sixty-five children with influenza demonstrated that children with H1N1 had significantly lower levels of CoQ10 compared to the group with seasonal influenza [23]. Supplementation with CoQ10 was found to reduce severity of viral myocarditis and reduce mortality in mice, likely through the reduction of oxidative stress [24]. Treatment with CoQ10 in hospitalized elderly patients with community-acquired pneumonia significantly reduced hospital stay and improved time to abatement of fevers [25].

While CoQ10 levels decrease with age and also through the consumption of exogenous agents such as statins, it has also been seen that several genetic diseases result in CoQ10 deficiencies. People with Down syndrome were found to have lower levels of CoQ10, and higher levels of TNFalpha and IL-6 [26]. Correspondingly, people with Down syndrome have a higher susceptibility to viral and bacterial infections, a higher incidence of autoimmune diseases (diabetes, hypothyroidism), and a higher incidence of acute lung injury [27]. Acute respiratory distress syndrome (ARDS) in people with Down syndrome has been postulated to be due to an imbalance in free radical scavengers [27]. Mutations of the CoQ genes can result in primary CoQ10 deficiency that is associated with low ATP production and the increased presence of ROS [11]. People with primary CoQ10 deficiency can have a range of clinical manifestations including encephalopathy, myopathy, and kidney disease, but many respond well to supplementation with CoQ10 to prevent further progression of disease [11]. 


\section{CoQ10 and COVID-19}

Understanding the etiology of why specific members of the population are more susceptible to severe disease necessitating hospitalization with SARS-CoV2 infection is important in the development of therapeutics, and may provide a rapid solution for this globally catastrophic pandemic. An examination of the epidemiological associations has demonstrated the occurrence of severe illness more widely in people of specific age groups possessing certain co-morbid conditions. Mortality and severity of illness of COVID-19 increases dramatically with increasing age [28]. The most common co-morbidities for hospitalized patients with COVID-19 are hypertension, diabetes, and obesity [28]. Although severe disease with COVID-19 often involves respiratory compromise with bilateral interstitial opacities on imaging; notably in the case of severe infection, pulmonary disease is less often a comorbidity [29].

An inverse correlation is seen with age and $\mathrm{BMI}$, as it appears that younger people who were hospitalized were more often obese [30]. Statins are among the most widely prescribed medications in the United States in 2020, and would likely be more commonly prescribed to patients with disorders such as hypertension, diabetes, and obesity, frequently seen with severe SARS-CoV2 infection [31]. If decreased levels of CoQ10 are determined to increase susceptibility to severe COVID-19 pneumonia, people may consider stopping statins in the short-term during the pandemic to reduce risk.

Further, the finding of increasing age being associated with lower CoQ10 levels correlates well with the connection between age and severe illness in COVID-19, as the disease significantly affects individuals of older age more often while causing mild illness in the vast majority of children [30]. The severe complications of COVID-19 including ARDS are thought to be due to a hyperinflammatory state [32]. Given the widespread role of CoQ10 in mitigating oxidative stress in the affected organ systems, interplay with inflammatory mediators, and the possibility that the demographic of severely affected people are at risk of CoQ10 deficiency, CoQ10 may be a marker of those susceptible to severe disease with COVID-19 and may be a causative agent for disease progression to the pathological hyper-inflammatory state (Figure 1).

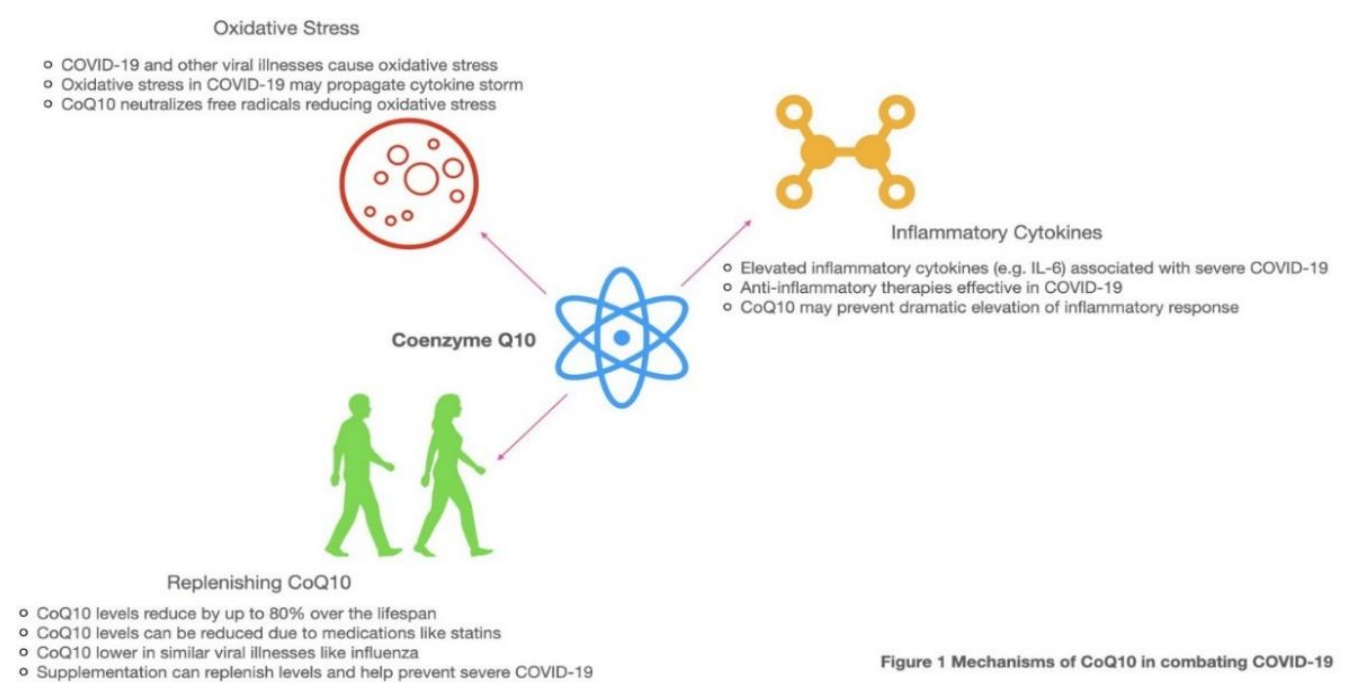

Figure 1 Mechanisms of CoQ10 in combating COVID-19: 1 Scavenging free radicals and reduction of oxidative stress; 2 Mitigation of damaging inflammatory cytokines; 3 Replenishing levels in aging individuals whose levels of CoQ10 decrease with age. 


\section{Exploring the Role of CoQ10 in COVID-19}

A potential association may exist between reduced levels of CoQ10 and the population of people most severely affected by COVID-19. The causative mechanisms of creating a susceptibility to severe illness remain unclear, though they could be a result of a reduced ability to preventing oxidative stress, attenuating coagulation, mitigating a hyper-immune response, or inhibiting viral replication or viral entry directly. The deficiencies associated with disease may involve other antioxidants such as Vitamin C and E. Exploring whether correlations exist between known antioxidants such as CoQ10 and inflammatory cytokines can help further understand the pathophysiologic process and find new therapeutic solutions.

Patients with influenza were found to have significantly lower levels of CoQ10 when compared to healthy controls [22]. Further, II-2 and TNF-alpha were found to be negatively correlated with levels of CoQ10 in influenza patients [22]. Large studies should continue to measure CoQ10 levels along with vitamins and lipids of infected people with COVID-19 at the time of presentation to examine whether correlations predict clinical outcomes and correlate with the levels of inflammatory cytokines and molecules such as IL-2, IL-6, TNF-alpha and D-dimer. Studies have demonstrated relatively higher levels of II-6 in patients with severe COVID-19 [33]. The cytokine storm hypothesis has prevailed as the leading hypothesis for the development of complications resulting in severe COVID-19, with a focus on IL-6 [34]. The evaluation of anti-inflammatory therapies has been investigated in patients with severe COVID-19, with corticosteroids showing significant therapeutic benefit and reduction in mortality [35]. Recently, the JAK1/2 inhibitor baricitinib, when combined with remdesivir, was shown to reduce median recovery time by one day [36]. As such, CoQ10, which mitigates the hyper-immune response may play an integral role in the susceptibility and development to severe COVID-19. Clinical outcomes of COVID-19 in individuals with genetically predisposed CoQ10 deficiency should also be examined.

Given the complexity of SARS-CoV2 infection and heterogeneity in disease presentation, the reasons for severe illness are likely multifactorial. CoQ10 may serve as a marker correlated to severe illness, and potentially as a causative agent for susceptibility to worse clinical outcomes.

\section{Conclusions}

If an association is confirmed, a causative mechanism could further be explored and CoQ10 may potentially become a protective therapy in the future. Studies using CoQ10 supplementation found that daily doses of between $100 \mathrm{mg}$ to $200 \mathrm{mg}$ significantly improved inflammatory markers [37]. Based on these results, dosing to replenish levels of CoQ10 in deficiency could be initiated between $100 \mathrm{mg}$ to $200 \mathrm{mg}$ daily to have physiological impact [37]. While supplementation with CoQ10 is generally well-tolerated, adverse effects have included gastrointestinal upset, abdominal discomfort, allergic reaction and headache [38]. As is the case with many disease states, more impactful benefits can be made when treatments are used as prophylaxis. If lower levels of CoQ10 are correlated with severe COVID-19 illness, supplementation of deficient individuals may potentially offer a therapeutic solution to reduce the burden of disease and improve the state of this pandemic. 


\section{Author Contributions}

VM Polymeropoulos completed all work for this manuscript.

\section{Funding}

This work was conducted without any funding.

\section{Competing Interests}

Author VM Polymeropoulos was employed by Vanda Pharmaceuticals.

\section{References}

1. Lee C. Therapeutic modulation of virus-induced oxidative stress via the nrf2-dependent antioxidative pathway. Oxid Med Cell Longev. 2018; 2018: 1-26

2. Ansar M, Ivanciuc $T$, Garofalo RP, Casola A. Increased lung catalase confers protection against experimental RSV infection. Sci Rep. 2020; 10: 3653.

3. Saini R. Coenzyme Q10: The essential nutrient. J Pharm Bioallied Sci. 2011; 3: 464-467.

4. Fakhrolmobasheri M, Hosseini MS, Shahrokh SG, Mohammadi Z, Kahlani MJ, Majidi SE, et al. Coenzyme Q10 and its therapeutic potencies against COVID-19 and other similar infections: A molecular review. Zenodo. 2020; 1: 3788046.

5. Banerjee A, Czinn SJ, Reiter RJ, Blanchard TG. Crosstalk between endoplasmic reticulum stress and anti-viral activities: A novel therapeutic target for COVID-19. Life Sci. 2020; 255: 117842.

6. Rundek T, Naini A, Sacco R, DiMauro S. Atorvastatin decreases the coenzyme Q10 level in the blood of patients at risk for cardiovascular disease and stroke. Arch Neurol. 2004; 61: 889-892.

7. El-Hattab AW, Adesina AM, Jones J, Scaglia F. MELAS syndrome: Clinical manifestations, pathogenesis, and treatment options. Mol Genet Metab. 2015; 116: 4-12.

8. Xu Y, Nisenblat V, Lu C, Rong L, Jie Q, Zhen XM, Wang SY. Pretreatment with coenzyme Q10 improves ovarian response and embryo quality in low-prognosis young women with decreased ovarian reserve: A randomized controlled trial. Reprod Biol Endocrinol. 2018; 16: 29.

9. Hargreaves IP, Mantle D. Coenzyme Q10 supplementation in fibrosis and aging. Adv Exp Med Biol. 2019; 1178: 103-112.

10. Kalen A, Appelkvist EL, Dallner G. Age-related changes in the lipid compositions of rat and human tissues. Lipids. 1989; 24: 579-584

11. Acosta JM, Fonseca LV, Desbats MA, Cerqua C, Zordan R, Trevisson E, et al. Coenzyme Q biosynthesis in health and disease. Biochem Biophys Acta. 2016; 1857: 1079-1085.

12. Li R, Ren T, Zeng J. Mitochondrial Coenzyme $Q$ protects sepsis-induced acute lung injury by activating PI3K/Akt/GSK-3ß/mTOR pathway in rats. Biomed Res Int. 2019; 2019: 5240898.

13. Donnino MW, Cocchi MN, Salciccioli JD, Kim D, Naini AB, Buettner C, et al. Coenzyme Q10 levels are low and may be association with the inflammatory cascade in septic shock. Crit Care. 2011; 15: R189.

14. Ya F, Xu XR, Shi Y, Gallant RC, Song F, Zuo X, et al. Coenzyme Q10 upregulates platelet cAMP/PKA pathway and attenuates integrin allb $\beta 3$ signaling and thrombus growth. Mol Nutr Food Res. 2019; 63: e1900662. 
15. Serebruany VL, Ordonoez JV, Herzog WR, Rohde M, Mortensen SA, Folkers K, et al. Dietary coenzyme Q10 supplementation alters platelet size and inhibits human vitronectin (CD51/CD61) receptor expression. J Cardiovasc Pharmacol. 1997; 29: 16-22.

16. Mohamed DI, Khairy E, Tawfek SS, Habib EK, Fetouh MA. Coenzyme Q10 attenuates lung and liver fibrosis via modulation of autophagy in methotrexate treated rat. Biomed Pharmacother. 2019; 109: 982-1001.

17. Farsi F, Mohammadshahi M, Alavinejad P, Rezazadeh A, Zarei M, Engali KA. Function of coenzyme Q10 supplementation on liver enzymes, markers of systemic inflammation, and adipokines in patients affected by nonalcoholic fatty liver disease: A double-blind, placebocontrolled, randomized clinical trial. J Am Coll Nutr. 2016; 35: 364-353.

18. Mortensen SA, Rosenfeldt F, Kumar A, Dolliner P, Filipiak JK, Pella D, et al. The effect of coenzyme Q10 on morbidity and mortality in chronic heart failure: Results from Q-SYMBIO: A randomized double-blind trial. JACC Heart Fail. 2014; 2: 641-649.

19. Soja AM, Mortensen SA. Treatment of chronic cardiac insufficiency with coenzyme Q10, results of meta-analysis in controlled clinical trials. Ugeskr Laeger. 1997; 159: 7302-7308.

20. Dludla PV, Nyambuya TM, Orlando P, Silvestri S, Mxinwa V, Mokgalaboni K, et al. The impact of coenzyme Q10 on metabolic and cardiovascular disease profiles in diabetic patients: A systematic review and meta-analysis of randomized controlled trials. Endocrinol Diabetes Metab. 2020; 3: e00118.

21. Gao L, Mao Q, Cao J, Wang Y, Zhou X, Fan L. Effects of coenzyme Q10 on vascular endothelial function in humans: A meta-analysis of randomized clinical trials. Atherosclerosis. 2012; 221: 311-316.

22. Chase M, Cocchi MN, Liu X, Andersen LW, Holmberg MJ, Donnino MW. Coenzyme Q10 in acute influenza. Influenza Other Respir Viruses. 2019; 13: 64-70.

23. Kelekçi S, Evliyaoğlu O, Yolbaş, Uluca U, Tan I, Gürkan MF. The relationships between clinical outcome and the levels of total antioxidant capacity (TAC) and coenzyme Q (CoQ 10) in children with pandemic influenza (H1N1) and seasonal flu. Eur Rev Med Pharmacol Sci. 2012; 16: 10331038.

24. Miyamoto S, Ito T, Terada S, Eguchi T, Furubeppu H, Kawamuraet $\mathrm{H}$, et al. Fulminant myocarditis associated with severe fever with thrombocytopenia syndrome: A case report. BMC Infect Dis. 2019; 19: 266.

25. Farazi A, Sofian M, Jabbariasl M, Nayebzadeh B. Coenzyme $q 10$ administration in communityacquired pneumonia in the elderly. Iran Red Crescent Med J. 2014; 16: e18852.

26. Zaki ME, El-Bassyouni HT, Tosson AM, Younness E, Hussein J. Coenzyme Q10 and proinflammatory markers in children with down syndrome: Clinical and biochemical aspects. J Pediatr. 2017; 93: 100-104.

27. Brujn $M$, van der Aa LB, van Rijn RR, Bos AP, van Woensel JB. High incidence of acute lung injury in children with down syndrome. Intensive Care Med. 2007; 33: 2179-2182.

28. Richardson S, Hirsch JS, Narasimhan M. Presenting characteristics, co-morbidities, and outcomes among 5700 patients hospitalized with COVID-19 in the New York City area. JAMA. 2020; 323: 2052-2059.

29. Zhou F, Yu T, Du RH, Fan GH, Liu Y, Liu ZB, et al. Clinical course and risk factors for mortality of adult inpatients with COVID-19 in Wuhan, China: A retrospective cohort study. Lancet. 2020; 395: 1054-1062. 
30. Kass DA, Duggal P, Cingolani O. Obesity could shift severe COVID-19 disease to younger ages. Lancet. 2020; 395: 1544-1545

31. Center for Disease Control and Prevention. National Health and Nutrition Examination Survey. Atlanta: CDC; 2019. Available From: https://www.cdc.gov/nchs/nhanes/index.htm.

32. Merad M, Martin JC. Pathological inflammation in patients with COVID-19: A key role for monocytes and macrophages. Nat Rev Immunol. 2020; 20: 335-362.

33. Mojtabavi H, Saghazadeh A, Rezaei N. Interleukin-6 and severe COVID-19: A systematic review and meta-analysis. Eur Cytokine Netw. 2020; 31: 44-49.

34. Tang Y, Liu J, Zhang D, Xu Z, Ji J, Wen C. Cytokine storm in COVID-19: The current evidence and treatment strategies. Front Immunol. 2020; 11: 1708.

35. WHO rapid evidence appraisal for COVID-19 therapies (REACT) working group. Association between administration of systemic corticosteroids and mortality among critically III patients with COVID-19: A meta-analysis. JAMA. 2020; 324: 1-13.

36. Lilly E. Baricitinib in combination with remdesivir reduces time to recovery in hospitalized patients with COVID-19 in NIAID-Sponsored ACTT-2 trial. Canada: GlobeNewswire; 2020. Available From: https://www.globenewswire.com/newsrelease/2020/09/17/2095439/0/en/Baricitinib-in-Combination-with-Remdesivir-ReducesTime-to-Recovery-in-Hospitalized-Patients-with-COVID-19-in-NIAID-Sponsored-ACTT-2-

Trial.html.

37. Dludla PV, Orlando P, Silvestri S, Marcheggiani F, Cirilli I, Nyambuya TM, et al. Coenzyme Q10 supplementation improves adipokine levels and alleviates inflammation and lipid peroxidation in conditions of metabolic syndrome: A meta-analysis of randomized controlled trials. Int J Mol Sci. 2020; 21: 3247.

38. Hidaka T, Fujii K, Funahashi I, Fukutomi N, Hosoe K. Safety assessment of coenzyme Q10 (CoQ10). Biofactors. 2008; 32: 199-208.

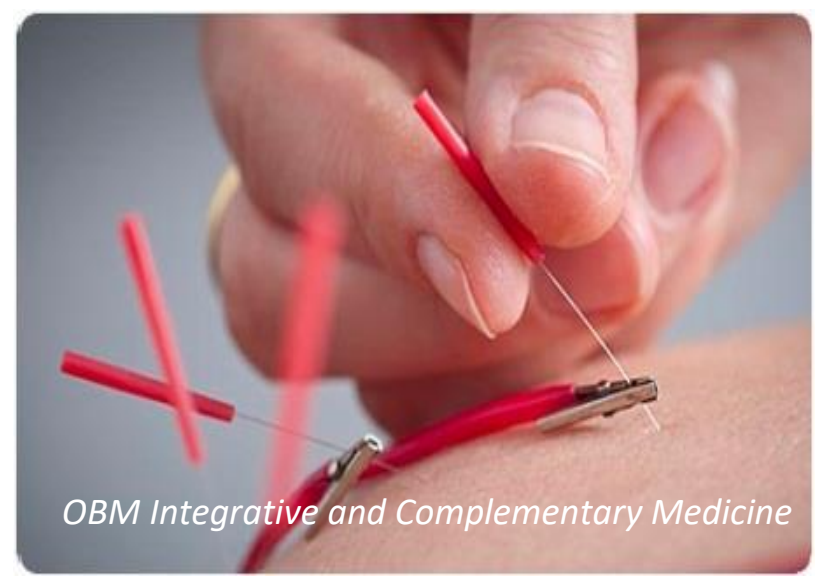

Enjoy OBM Integrative and Complementary Medicine by:

1. Submitting a manuscript

2. Joining in volunteer reviewer bank

3. Joining Editorial Board

4. Guest editing a special issue

For more details, please visit: http://www.lidsen.com/journals/icm 
Editorial

\title{
Dynamic Pulse Reaction - Update 2020
}

\section{Gerhard Litscher *}

Research Unit of Biomedical Engineering in Anesthesia and Intensive Care Medicine, Research Unit for Complementary and Integrative Laser Medicine, and Traditional Chinese Medicine (TCM) Research Center Graz, Medical University of Graz, 8036 Graz, Austria; E-Mail: gerhard.litscher@medunigraz.at

* Correspondence: Gerhard Litscher; E-Mail: gerhard.litscher@medunigraz.at

Collection: Trends in Acupuncture and Laser Research and Education

OBM Integrative and Complementary Medicine

2020 , volume 5 , issue 4

doi:10.21926/obm.icm.2004043
Received: November 11, 2020

Accepted: November 12, 2020

Published: November 13, 2020

\begin{abstract}
The Nogier reflex or reflex auriculo-cardiac (RAC) or the dynamic pulse reaction is a physiological phenomenon that is not generally accepted in conventional medicine. Therefore, evidence-based research in this sub-area is important for auricular medicine as it provides appropriate scientific evidence for better acceptance. In this editorial, a RAC measurement based on a modified smartphone registration and analysis is presented. The contribution is intended to stimulate researchers to continue working in this interesting area.

\section{Keywords}

Nogier reflex; reflex auriculo-cardiac (RAC); dynamic pulse reaction; auricular medicine; basic research; integrative and complementary medicine
\end{abstract}

\section{Introduction}

The importance of the Nogier reflex or reflex auriculo-cardiac (RAC) or the dynamic pulse reaction as a physiological variable that has so far been almost unknown in conventional medicine

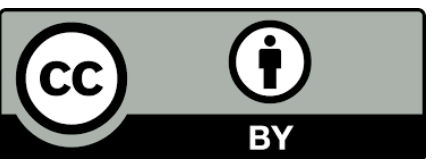

(C) 2020 by the author. This is an open access article distributed under the conditions of the Creative Commons by Attribution License, which permits unrestricted use, distribution, and reproduction in any medium or format, provided the original work is correctly cited. 
can be regarded as considerable [1]. It is a dynamic change [1]. Usually, we feel the RAC while microstimuli are acting on the patient, which is not a static process. We usually only feel it with a fingertip. There is only one ideal pulse sampling point (in an anatomically predetermined position). The RAC palpation provides reproducible results in double-blind tests [1].

In a (2019/2020) multinational research project of the TCM (Traditional Chinese Medicine) Research Center at the Medical University of Graz funded by IMON (Initiative for Medicine without Side Effects), efforts were made to break new ground, primarily providing quantifiable evidence for the changes in dynamic pulse recording with optical methods and artificial intelligence. The results were registered for the first time using an adapted smartphone and can be used for further research by other groups.

Detailed technical descriptions can be found in a report that was already presented to a broad interest group [2].

\section{Information Technology-Based Dynamic 'Pulse Sensing' Using an Adapted Smartphone}

The 'dynamic pulse phenomenon' described by Bahr [1] was first described by Paul Nogier in the 1970s [3-5]. Based on the assumption that most of the cells of an organism react to stimuli from the autonomic nervous system, Nogier looked for a method with which the body's reaction to acupuncture-induced measures could be represented. He discovered that mechanical pressure on certain points on the auricle caused a cardiovascular reaction. He called this reaction 'reflexe auriculo cardiaque', later it was also abbreviated as RAC or, as Bahr preferred, called 'dynamic pulse reaction' (to differentiate it from Chinese static pulse sensing) [1]. In the research of Bahr et al., the RAC served for its practical application in the field of auriculomedicine both as an instrument for diagnosis and therapy [6]. The use of the RAC signal enabled the development of interference field acupuncture as well as the therapeutic so-called 'controlled acupuncture', in which the acupuncture needle is positioned under dynamic pulse control and not, as in the classical Chinese tradition, based on 'cun' and other units of measurement [6]. Litscher et al. [7] published an original article in Integrative Medicine International in 2014 together with German and Chinese researchers. A new high-resolution imaging technique for registering pulsating surface changes could make it possible to quantify the RAC reproducibly for the first time even without statistical analysis. This method combines an innovative microscope system, video analysis software, and special image processing software (from the Beijing University of Science and Technology). Even small, pulse-dependent changes in the skin surface could be made visible [7]. Moser et al. [8], also from the Medical University of Graz in Austria, reported in 2017 that the RAC follows the physiological laws quite well. The study, based on an earlier set of tests on measurements and analyses of heart rate variability, showed various reproducible changes in physiological parameters. A total of seven parameters and various external non-invasive stimuli were recorded under a defined setup and test course. The authors assumed that the RAC is a manifestation of a cardiac reaction of the autonomic nervous system, which then triggers a pulse wave. They concluded that the use of subtle stimuli near the limit of perception should be appropriate to allow for repeatable testing of immediate vagal and delayed sympathetic response.

The application presented in this editorial is based, for the first time, on a modified smartphone registration of pulse activity and can be applied to both static and dynamic pulse reactions. 
The hardware architecture of the innovative system is divided into three parts, the sensors, the smartphone per se, and the cloud server section. The sensors first register GSR (galvanic skin response) and PPG (photoplethysmography) signals from the patient and transfer them to the smartphone. The mobile unit then transmits the data wirelessly to the cloud server. The RAC is predicted with the help of artificial neural networks [9].

Figure 1 shows the dynamic pulse response (RAC) after triggering by laser stimulation at the Shenmen ear point $(810 \mathrm{~nm}, 150 \mathrm{~mW})$. The changes in amplitude and frequency in the pulse signal of the radial artery can be seen.

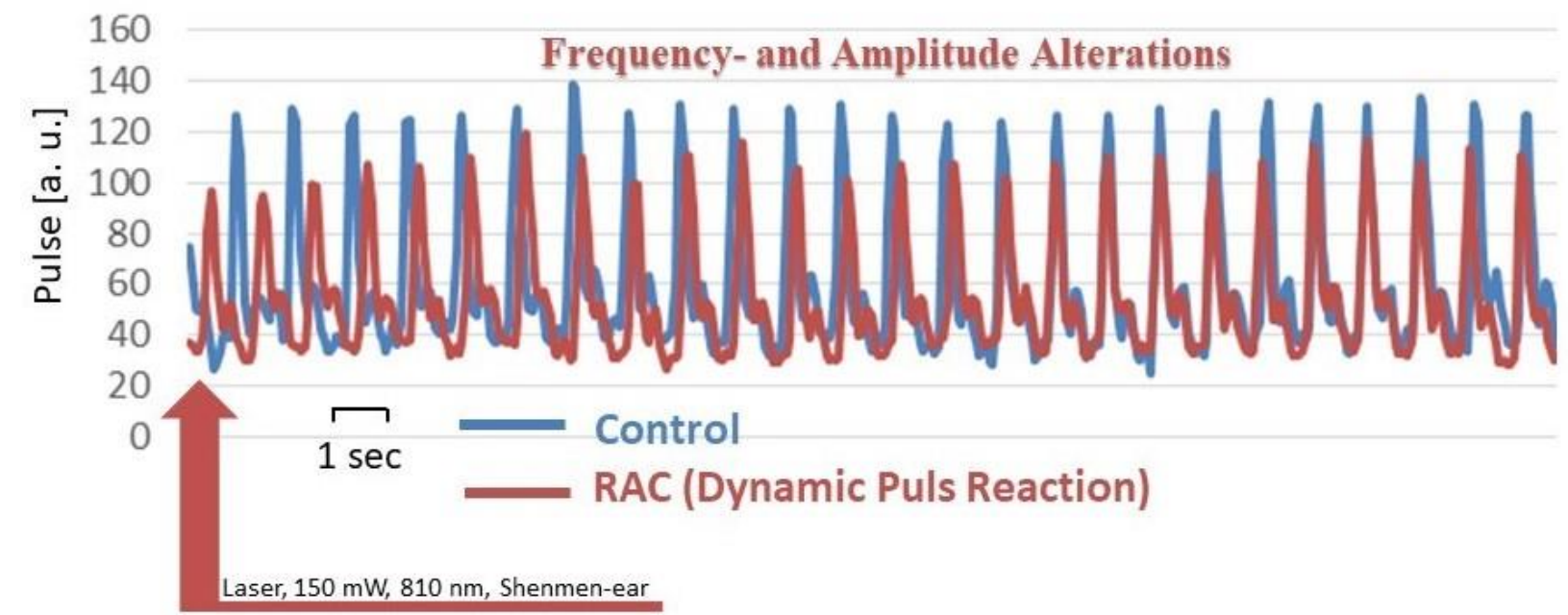

Figure 1 Dynamic pulse response (RAC) triggered by laser stimulation at the Shenmen ear point (modified with permission from Lan KC, Tainan).

\section{Future Aspects}

RAC research is entering a new phase in which modern information technology and artificial intelligence (AI) will play a major role. The RAC could be quantified and documented in the future using adapted smartphones with integrated sensors and analysis methods of Al (neural networks).

In theory, Al will help in making better medical decisions by registering, storing, and analyzing RAC data. However, in practice, it is often difficult to convert the changes in heart rate into usable information. The requirements of medical professionals are different, and the influencing factors too diverse.

Today, we are already taking the opposite step, and the human factor is again playing a bigger role. From the author's point of view, the greatest difficulty is to include human perception in the RAC analysis process. The solution to these questions can only be that $\mathrm{Al}$ and information technology processes in RAC research cannot and should not be a substitute for the human factor, but the basis for its data-supported decision-making.

\section{Acknowledgments}

The author of this editorial would like to thank all members of the Initiative for Medicine without Side Effects (IMON, non-profit association), the European Academy of Traditional Chinese Medicine (EATCM), and especially its president Professor Frank Bahr for the support made to the Medical 
University of Graz. Many thanks also to the project partners and employees from Austria, China and regions of China, especially Professor Kun-Chan Lan, PhD, Department of Computer Science and Information Engineering, National Cheng Kung University, Tainan, Taiwan and Priv.-Doz. Lu Wang, MD LAc, Research Unit for Complementary and Integrative Laser Medicine, Research Unit of Biomedical Engineering in Anesthesia and Intensive Care Medicine, and TCM Research Center at the Medical University of Graz, Austria. Parts of this editorial will be published in modified form as a short report in German language in Akupunktur \& Aurikulomedizin in vol.4, December 2020 [10].

\section{Author Contributions}

Gerhard Litscher did all work.

\section{Funding}

IMON/EATCM, Germany and TCM Research Center Graz, Medical University of Graz.

\section{Competing Interests}

The author does not declare any conflict of interest.

\section{References}

1. Bahr F. Personal communication 8.7. und 22. 10. 2020.

2. Litscher G. Dynamic pulse reaction and auricular medicine. Preliminary results from innovative transcontinental developments and basic research. Project report including 10 peer reviewed manuscripts. Graz: TCM Research Center Graz; 2020.

3. Nogier P. Le Réflexe Auriculocardiaque (RAC). Paris: Lyon Méditeranée Medical; 1972. p. 1709p. 1738.

4. Nogier R. How did Paul Nogier establish the map of the ear? Med Acupunct. 2014; 26: 76-83.

5. Oleson T. Auriculotherapy stimulation for neuro-rehabilitation. NeuroRehabilitation. 2002; 17: 49-62.

6. Bahr F, Strittmatter B. Das große Buch der Ohrakupunktur. Stuttgart: Hippokrates; 2014.

7. Litscher G, Bahr F, Litscher D, Min LQ, Rong PJ. A new method in auricular medicine for the investigation of the Nogier reflex. Integr Med Int. 2014; 1: 205-210.

8. Moser M, Frühwirth M, Messerschmidt D, Goswami N, Dorfer L, Bahr F, et al. Investigation of a micro-test for circulatory autonomic nervous system responses. Front Physiol. 2017; 8: 448.

9. Lan KC, Litscher G, Hung TH. Traditional Chinese medicine pulse diagnosis on a smartphone using skin impedance at acupoints: A feasibility study. Sensors. 2020; 20: 4618.

10. Litscher G. Ein Projektkurzbericht zur dynamischen Pulsreaktion in COVID-19-Pandemie-Zeiten. Akupunktur Aurikulomedizin. 2020. 
OBM Integrative and Complementary Medicine 2020; 5(4), doi:10.21926/obm.icm.2004043

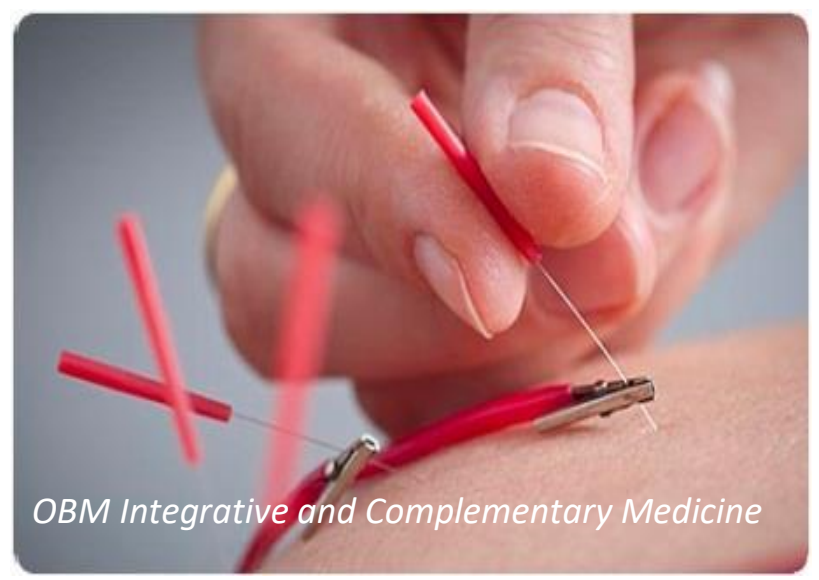

Enjoy OBM Integrative and Complementary Medicine by:

1. Submitting a manuscript

2. Joining in volunteer reviewer bank

3. Joining Editorial Board

4. Guest editing a special issue

For more details, please visit:

http://www.lidsen.com/journals/icm 
Review

\title{
Nutritional Interventions in Cancer Cachexia Prevention and Treatment
}

Wangkuk Son ${ }^{\dagger}$, Jason Lin $^{\dagger}$, Melissa Puppa ${ }^{*}$

University of Memphis College of Health Sciences, 495 Zach Curlin St., Memphis, TN, USA; E-Mails: wangkuk.s@memphis.edu; jlin5@memphis.edu; mpuppa@memphis.edu.

+ These authors contributed equally to this work.

* Correspondence: Melissa Puppa; E-Mail: mpuppa@memphis.edu

Academic Editor: Gerhard Litscher

Special Issue: $\underline{\text { Cancer Cachexia }}$

OBM Integrative and Complementary Medicine

2020 , volume 5 , issue 4

doi:10.21926/obm.icm.2004044
Received: September 28, 2020

Accepted: Novemeber 23, 2020

Published: December 7, 2020

\begin{abstract}
Cancer cachexia contributes to $30 \%$ of cancer-related deaths. There is currently no treatment or standard of care for cancer cachexia. Many nutritional interventions show promise for the treatment and or prevention of cachexia. Supplementation with omega-3 fatty acids, protein and vitamins either alone or in combination has shown some beneficial effects in the prevention and treatment of cancer cachexia. The mechanisms through which many nutritional interventions work to attenuate cachexia are just beginning to be understood. Therefore, the purpose of this review is to examine several nutritional strategies that have been investigated in the prevention and or treatment of cancer cachexia and provide evidence for the use of additional nutritional interventions to combat cachexia.
\end{abstract}

\section{Keywords}

Cachexia; nutrition; omega-3 PUFA; protein supplementation; probiotics; fasting; ketogenic; vitamin D

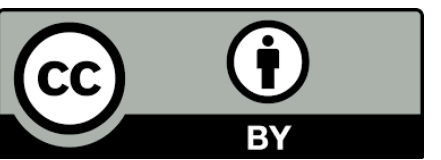

(C) 2020 by the author. This is an open access article distributed under the conditions of the Creative Commons by Attribution License, which permits unrestricted use, distribution, and reproduction in any medium or format, provided the original work is correctly cited. 


\section{Introduction}

Cancer induced cachexia, the unintentional loss of body weight and muscle mass, occurs in approximately $80 \%$ of cancer patients and contributes to $30 \%$ of cancer-related deaths [1]. While cachexia does not occur with all cancer, it is especially prominent in colorectal, lung, and pancreatic cancers. The cachexia stage correlates with patient morbidity and mortality and the cancer treatments $[2,3]$. The loss of skeletal muscle and adipose as well as the metabolic dysfunction and systemic inflammatory environment that occur with cachexia leads to decreased responsiveness to therapies and eventually, poorer prognosis [4-6].

Cachexia is a multifactorial process that includes metabolic dysfunction, systemic inflammation, hypogonadism, and anemia [6, 7]. As there are both tumor derived factors and alterations in the immune system that contribute to muscle and adipose catabolism, it is difficult to understand how to treat this condition best. While the prevention of cachexia has been demonstrated with exercise [8], challenges arise. Most patients are not diagnosed with cachexia until they have significant weight loss, at which point they experience fatigue, and exercise interventions may not be feasible [9]. Hence, treatment strategies become as important as prevention to improve cancer patients' treatment efficacy, quality of life, and survivorship [6]. There is currently no treatment for cachexia, and the target of most treatment is the tumor itself. Therefore, the use of nutritional interventions to prevent and treat cancer cachexia is gaining popularity.

A number of studies conducted in the 1980 s attempted to use parenteral nutrition in the treatment and management of cancer cachexia $[10,11]$. These studies demonstrated that increased caloric consumption alone was insufficient to attenuate cachexia and did not offer benefits in treating the underlying cancer. Since then, several additional studies utilizing parenteral nutrition have been conducted with similar results. Patients receiving parenteral nutrition experienced more severe adverse events, and there was no improvement in the quality of life [12]. More recent studies using enteral nutrition show more promise and have been shown to improve nutritional status and lower post-operative complications than parenteral nutrition in cancer patients [13]. Additional nutritional interventions have been studied in the context of cancer cachexia that are more targeted toward some of the known underlying mediators of cancer cachexia, including omega-3 supplementation [14], protein supplementation [15], and pro/prebiotics [16, 17]. The purpose of this review is to highlight several nutritional interventions and their potential for the prevention and treatment of cancer cachexia.

\section{Methods}

A comprehensive search of peer-reviewed journals was completed on a wide range of key terms including but not limited to cachexia, nutrition, omaga-3, vitamin D, fasting, time restricted feeding, protein, ketogenic, and probiotics were searched using the NCBI and Google Scholar databases. Only peer reviewed research was used. While there are other nutritional interventions that may aid in the prevention or treatment of cancer cachexia, the topics of omega-3 fatty acids, protein, pre/probiotics, vitamin $D$, fasting, and the ketogenic diet were selected for this targeted review. Table 1 gives an overview of relevant clinical studies included in this review. 
Table 1 Review of clinical studies.

\begin{tabular}{|c|c|c|c|c|c|c|}
\hline Reference & Subjects & Study Design & Intervention & Measurements & Outcomes & Principal Finding \\
\hline $\begin{array}{l}\text { Laviano et } \\
\text { al. (2020) }\end{array}$ & $\begin{array}{l}\text { Patients with non- } \\
\text { small-cell lung } \\
\text { cancer (NSCLC) } \\
\text { receiving first-line } \\
\text { chemotherapy }\end{array}$ & $\begin{array}{l}\text { 1. Targeted medical } \\
\text { nutrition (TMN) } \\
\text { (Whey protein+n- } \\
\text { 3PUFA+VitaminD3) } \\
\text { 2. Isocaloric } \\
\text { comparator }\end{array}$ & $\begin{array}{l}\text { Juice-based TMN } \\
\text { drink or milk-based } \\
\text { isocaloric } \\
\text { comparator drink } \\
\text { for } 12 \text { weeks, daily. }\end{array}$ & $\begin{array}{l}\text { Adverse events } \\
\text { (AES) Vital sign } \\
\text { laboratory safety } \\
\text { parameters } \\
\text { Compliance with } \\
\text { nutrition Exercise } \\
\text { capacity Survival } \\
\text { over } 12 \text { months } \\
\text { Clinical measures }\end{array}$ & $\begin{array}{l}\text { TMN vs Comparator } \\
\text { Smaller number of } \\
\text { AES ( } 64 \text { vs } 87 \text { ) Fewer } \\
\text { compliance ( } 58.5 \% \text { vs } \\
73.6 \% \text { ) Exercise } \\
\text { capacity favored } \\
\text { TMN Smaller } \\
\text { patients died (4 vs } \\
\text { 10) }\end{array}$ & $\begin{array}{l}\text { TMN containing high } \\
\text { dose n3 PUFA, vitamin } \\
\text { D and high-quality } \\
\text { protein chemotherapy } \\
\text { tolerability and has a } \\
\text { favorable safety profile. }\end{array}$ \\
\hline $\begin{array}{l}\text { Del Fibro et } \\
\text { al. (2013) }\end{array}$ & $\begin{array}{l}\text { Advanced cancer } \\
\text { patient with } \\
\text { decreased weight } \\
\text { and appetite }\end{array}$ & $\begin{array}{l}\text { 1. Fish oil } \\
\text { 2. Placebo }\end{array}$ & $\begin{array}{l}\text { Daily high doses of } \\
\text { fish oil capsules or } \\
\text { placebo (olive oil) } \\
\text { for } 2 \text { weeks }\end{array}$ & $\begin{array}{l}\text { Appetite Tiredness } \\
\text { Nausea } \\
\text { Well-being Caloric } \\
\text { Intake Nutritional } \\
\text { Status Function }\end{array}$ & $\begin{array}{l}\text { No improvement in } \\
\text { all of measures in } \\
\text { Fish oil group } \\
\text { compared to placebo }\end{array}$ & $\begin{array}{l}\text { Fish oil did not } \\
\text { influence appetite, } \\
\text { tiredness, nausea, well- } \\
\text { being, caloric intake, } \\
\text { nutritional status, or } \\
\text { function after } 2 \text { weeks } \\
\text { compared with placebo }\end{array}$ \\
\hline $\begin{array}{l}\text { Murphy et } \\
\text { al. (2011) }\end{array}$ & $\begin{array}{l}\text { Patient with non- } \\
\text { small cell lung } \\
\text { cancer (NSCLC) } \\
\text { receiving first-line } \\
\text { chemotherapy }\end{array}$ & $\begin{array}{l}\text { 1. Fish oil } \\
\text { 2. Standard Care }\end{array}$ & $\begin{array}{l}\text { Daily fish oil } \\
\text { capsules or } \\
\text { standard care for } 10 \\
\text { weeks }\end{array}$ & $\begin{array}{l}\text { Weight } \\
\text { Skeletal muscle } \\
\text { Adipose tissue }\end{array}$ & $\begin{array}{l}\text { Fish oil ( } 69 \% \\
\text { patients) gained or } \\
\text { maintained muscle } \\
\text { mass and weight } \\
\text { compared with } 29 \% \\
\text { of Standard care }\end{array}$ & $\begin{array}{l}\text { Early intervention with } \\
\text { fish oil during } \\
\text { chemotherapy resulted } \\
\text { in maintenance of } \\
\text { weight and muscle } \\
\text { mass compared with }\end{array}$ \\
\hline
\end{tabular}


Patients with non-

Murphy et

al. (2010) small-cell lung cancer (NSCLC) receiving first-line chemotherapy

Barber et

al. (2004)

\section{Weight-losing} patients with pancreatic cancer

Time trial repeated measures with one group
1. Sarcopenic patient with NSCLC 2. Non-sarcopenic patient with NSCLC
Muscle mass Plasma phospholipid(n-3) fatty acid day

Oral n-3 fatty-acid- Hepatic synthesis enriched with fish of albumin oil supplement for 3 Fibrinogen Body weeks

n-3 fatty acid with antioxidant enriched oral

Cachectic patients 1. n-3 experiment with advanced pancreatic cancer

\section{(E)}

2. Control (C) al. (2003) supplement (E) or supplement without n-3 fatty acid with enhanced antioxidants (C) for 8 weeks
Sarcopenic patients had lower plasma EPA, DHA, and n-3 fatty acid than nonsarcopenic patients.

n-3 fish oil decreased albumin and fibrinogen synthetic rate in the fasting and fed state. Also, patient' weight stabilized

Both stopped losing weight and lean body mass. But E patients only have significant correlations between their supplement

intake, weight gain and lean body mass. patients receiving Standard care.

Plasma (n-3) fatty acids are depleted in cancer patients with sarcopenia, which may accelerate rates of muscle loss

Hepatic protein kinetics were modulated to some degree by an $n-3$ fatty-acid-enriched fish oil supplement.

If taken in sufficient quantity, only the $n-3$ enriched and protein dense supplement results in net gain of weight, lean tissue, and improved quality of life 


\begin{tabular}{|c|c|c|c|c|c|c|}
\hline \multirow[b]{2}{*}{$\begin{array}{l}\text { Wigmore et } \\
\text { al. (2000) }\end{array}$} & \multirow[b]{2}{*}{$\begin{array}{l}\text { Weight-losing } \\
\text { patients with } \\
\text { advanced } \\
\text { pancreatic cancer }\end{array}$} & \multirow[b]{2}{*}{$\begin{array}{l}\text { Time trial repeated } \\
\text { measures with one } \\
\text { group }\end{array}$} & \multirow[b]{2}{*}{$\begin{array}{l}\text { Oral } \\
\text { supplementation } \\
\text { with high-purity EPA } \\
\text { starting at } 1 \mathrm{~g} / \text { day to } \\
6 \mathrm{~g} / \text { day for } 12 \text { weeks }\end{array}$} & \multicolumn{3}{|l|}{ Tolerance/Toxicity } \\
\hline & & & & $\begin{array}{l}\text { Body composition } \\
\text { Hematologic and } \\
\text { clinical chemistry } \\
\text { Acute-phage } \\
\text { protein response } \\
\text { Performance status } \\
\text { Overall survival }\end{array}$ & $\begin{array}{l}\text { After EPA, weight } \\
\text { was stable. }\end{array}$ & $\begin{array}{l}\text { EPA is well tolerated, } \\
\text { may stabilize weight in } \\
\text { cachectic pancreatic } \\
\text { cancer patients }\end{array}$ \\
\hline $\begin{array}{l}\text { Barber et } \\
\text { al. (1999) }\end{array}$ & $\begin{array}{l}\text { Patients with } \\
\text { unresectable } \\
\text { pancreatic } \\
\text { adenocarcinoma, } \\
\text { ongoing weight- } \\
\text { loss }\end{array}$ & $\begin{array}{l}\text { Time trial repeated } \\
\text { measures with one } \\
\text { group }\end{array}$ & $\begin{array}{l}\text { Combination of EPA } \\
\text { with a conventional } \\
\text { oral nutritional } \\
\text { supplement per day } \\
\text { for } 7 \text { weeks }\end{array}$ & $\begin{array}{l}\text { Body composition } \\
\text { Dietary intake } \\
\text { Resting energy } \\
\text { expenditure (REE) } \\
\text { Performance status }\end{array}$ & $\begin{array}{l}\text { Weight-gain } \\
\text { Increased dietary } \\
\text { intake } \\
\text { Improved } \\
\text { performance status }\end{array}$ & $\begin{array}{l}\text { An EPA-enriched } \\
\text { supplement may } \\
\text { reverse cachexia in } \\
\text { advanced pancreatic } \\
\text { cancer }\end{array}$ \\
\hline $\begin{array}{l}\text { Cereda et } \\
\text { al. (2019) }\end{array}$ & $\begin{array}{l}\text { Malnourished } \\
\text { advanced cancer } \\
\text { patients } \\
\text { undergoing } \\
\text { chemotherapy }\end{array}$ & $\begin{array}{l}\text { 1. Only nutritional } \\
\text { counseling } \\
\text { (Control) } \\
\text { 2. Nutritional } \\
\text { counseling with } \\
\text { whey protein (WPI) }\end{array}$ & $\begin{array}{l}\text { Whey protein } \\
\text { isolate } \\
\text { supplementation } \\
\text { (WPI) in addition to } \\
\text { nutritional } \\
\text { counseling for } 3 \\
\text { months }\end{array}$ & $\begin{array}{l}\text { Change in phase } \\
\text { angle (PhA) } \\
\text { Standardized PhA } \\
\text { (SPA) } \\
\text { Fat-free mass index } \\
\text { (FFMI) } \\
\text { Body weight } \\
\text { Muscle strength } \\
\text { CT toxicity }\end{array}$ & $\begin{array}{l}\text { WPI improved PhA, } \\
\text { SPA, FFMI, body } \\
\text { weight, muscle } \\
\text { strength and a } \\
\text { reduced risk of CT } \\
\text { toxicity }\end{array}$ & $\begin{array}{l}\text { WPI may lead to } \\
\text { improved treatment } \\
\text { efficacy for } \\
\text { malnourished advanced } \\
\text { cancer patients }\end{array}$ \\
\hline $\begin{array}{l}\text { Tozer et al. } \\
(2008)\end{array}$ & $\begin{array}{l}\text { Patients with } \\
\text { stage IIIB-IV non- } \\
\text { small cell lung } \\
\text { cancer }\end{array}$ & $\begin{array}{l}\text { 1. Cysteine-rich } \\
\text { protein (IMN1207) } \\
\text { 2. Casein }\end{array}$ & $\begin{array}{l}\text { Oral administration } \\
\text { of the cysteine-rich } \\
\text { protein or casein for } \\
6 \text { months }\end{array}$ & $\begin{array}{l}\text { Overall compliance } \\
\text { Body weight } \\
\text { Survival and } \\
\text { Plasma amino acids }\end{array}$ & $\begin{array}{l}\text { Patients with } \\
\text { IMN1207 had an } \\
\text { increased body } \\
\text { weight, body cell }\end{array}$ & $\begin{array}{l}\text { The cysteine-rich } \\
\text { protein is sufficient to } \\
\text { reverse cancer-related } \\
\text { weight loss and the loss }\end{array}$ \\
\hline
\end{tabular}




\section{Storck et al. Advanced cancer (2020) patient \\ 1. Intervention \\ 2. Control}

$\begin{array}{ll}\begin{array}{l}\text { Patients with } \\ \text { advanced }\end{array} & \\ \begin{array}{ll}\text { esophageal cancer } & \text { 1. Synbiotics } \\ \text { receiving } & \text { 2. Control } \\ \text { neoadjuvant } & \\ \text { chemotherapy } & \end{array}\end{array}$

Hand-grip force

Quality of life

\section{A leucine-rich}

supplement in

combination with a

nutrition and

physical exercise

program for 12

weeks

\section{Oral synbiotics}

(Bifidobacterium,

Lactobacillus, and

galacto-

oligosaccharides) or

Biofermin

(Streptococcus

faecalis) treatment

for 6 weeks mass, hand-grip

force, and quality of

life

In inter-group, increased handgrip strength and a trend toward an improvement in nutritional status, dietary intake, fatigue, QoL and clinical course

In synbiotic groups, smaller frequencies

\section{of severe}

lymphopenia, febrile neutropenia and diarrhea. The syngroup improved the numbers of beneficial and harmful bacteria of body cell mass which is associated with an improvement of muscle force and certain quality of life parameters.

A multimodal therapy combining a leucinerich supplement with nutritional counselling and exercise is widely accepted by advanced cancer patients, more importantly improved handgrip strength Significantly

\section{Synbiotics during} chemotherapy reduce the occurrence of adverse events of chemotherapy through adjustments to the intestinal microbiota 
Patients with

Osterlund colorectal cance

et al. (2007) receiving

chemotherapy

Advanced non-

Akiba et al. small cell lung

(2018)

cancer (NSCLC)

patients

Patients with

advanced

1. Vitamin D

2. Placebo

metastatic

$\mathrm{Ng}$ et al.

colorectal cancer

receiving

chemotherapy

1. High-does

Vitamins
Lactobacillus.

rhamnosus GG or

fiber-containing

nutritional support

for 24 weeks

A does of 1,200

IU/day for 12

months

\section{Every}

chemotherapy cycle

(14days). The high

group $>4000 \mathrm{IU} / \mathrm{d}$

the standard $>$

400IU/d until

disease progression,

intolerance toxicity,

or decision to
Relapse-free

survival (RFS)

Overall survival

(OS) Serum levels

of 25(OH)D

Genotyping

associated-Vitamin

2. Standard-dose

Progression-free

survival (PFS)

Tumor objective

response rate

Grade 3 and

adverse events

Overall survival

(OR) Change in

plasma 25D level

In Lacto-GG showed

less grade 3 or 4

diarrhea, abdominal

discomfort, hospital

care and fewer

chemotherapy does

reduction due to

bowel toxicity

(Vitamin vs Placebo)

Relapse and Death

( $28 \%$ vs $17 \%$ ) By

restricting to

subgroup with early-

stage

5 -year RFS (86\% vs

$50 \%)$

OS ( $91 \%$ vs $48 \%)$

Lactobacillus GG

supplementation is well

tolerated and reduce

the frequency of severe

diarrhea and abdominal

discomfort due to

chemotherapy

\section{Vitamin D}

supplementation may

improve survival of

patients with early-

stage lung

adenocarcinoma with

lower 25D levels, but

not advanced stage

High-does vitamin

showed higher

median PFS and

improved supportive

hazard ratio

Higher plasma 25-

hydroxyvitamin D levels

are associated with

improved survival in

metastatic colorectal

cancer 


\begin{tabular}{|c|c|c|c|c|c|c|}
\hline & & & $\begin{array}{l}\text { discontinue } \\
\text { treatment }\end{array}$ & & & \\
\hline $\begin{array}{l}\text { Fearon et } \\
\text { al. (1988) }\end{array}$ & $\begin{array}{l}\text { Cachectic cancer } \\
\text { patients with } \\
\text { severe weight loss }\end{array}$ & $\begin{array}{l}\text { One group } \\
\text { 1. Normal diet } \\
\text { followed by } \\
\text { 2. Ketogenic diet }\end{array}$ & $\begin{array}{l}\text { Normal diet on days } \\
1-6 \text { Ketogenic diet } \\
\text { on days } 7-13\end{array}$ & $\begin{array}{l}\text { Blood substrates } \\
\text { Whole-body } \\
\text { protein turnover } \\
\text { Insulin } \\
\text { concentration Urea } \\
\text { and Electrolytes } \\
\text { Liver function test }\end{array}$ & $\begin{array}{l}\text { After ketogenic diet, } \\
\text { Ketosis blood of } \\
\text { glucose, lactate, and } \\
\text { pyruvate } \\
\text { Body mass } \\
\text { No significant } \\
\text { alteration in N } \\
\text { balance or whole- } \\
\text { body protein } \\
\text { synthesis, } \\
\text { degradation, or } \\
\text { turnover rates }\end{array}$ & $\begin{array}{l}\text { Whether the change } \\
\text { from glucose to fat- } \\
\text { derived energy } \\
\text { substrates might reduce } \\
\text { tumor growth rates in } \\
\text { the long term remains } \\
\text { to be determined }\end{array}$ \\
\hline
\end{tabular}




\section{Omega-3 Polyunsaturated Fatty Acids}

There are three types of omega-3 polyunsaturated fatty acids (PUFAs); eicosapentaenoic acid (EPA), docosahexaenoic acid (DHA), and alpha-linolenic acid (ALA). EPA and DHA are primarily found in a marine-based source such as fish and krill, while ALA is typically found in plant-based sources such as flax seed. ALA is a precursor to EPA and DHA and is converted albeit not efficiently in humans into EPA and DHA. Omega-3 has known beneficial health effects due to its anti-inflammatory properties and role in cell signaling and membrane fluidity. Omega-3 PUFAs, specifically EPA and DHA, target multiple systems and pathways that are dysregulated during cancer cachexia. There is an imbalance between protein synthesis and degradation during cachexia, leading to unregulated activation of degradation pathways including autophagy and ubiquitin proteasomal degradation, while inhibiting protein synthesis $[1,18]$. Concomitantly, there is also a loss of functional energyproducing mitochondria [19]. Much of this imbalance originates with the inflammatory environment (increased IL-6 or TNF- $\alpha$ ) induced by cancer [20]. Omega -3 PUFAs have been shown to be beneficial in preventing lean body mass loss with cancer [20,21].

Omega-3 PUFAs can modulate pro-inflammatory cytokines production, thereby altering the catabolic stimuli driving cachexia. Independent from its anti-inflammatory function, EPA inhibits cancer-induced protein degradation by inhibiting ATP-dependent proteolytic pathways [22, 23]. EPA has been shown to inhibit protein degradation by suppression of prostaglandin E2 (PGE2) and proteolysis-inducing factors (PIF) in muscle $[22,24-26]$. Many cachectic patients develop anabolic resistance with decreased protein synthesis pathways, even when given an anabolic stimulus [16]. By administering omega-3 PUFAs, the blockade of the anabolic response may be reduced and allow for muscle mass maintenance, which has been shown to correlate with cancer survivorship and quality of life, without promoting tumor growth [27, 28].

Fearon et al. demonstrated that cachectic patients have low plasma concentrations of EPA and DHA, so supplementation may prove beneficial [14]. Many studies have shown promising effects of omega-3 PUFA supplementation in advanced cancer patients in helping to stabilize weight [29, 30], attenuate muscle loss [30-33], and increase survival [32, 34]. Wigmore et al. evaluated 26 patients with pancreatic induced cachexia. After increasing the dose over the course of 4 weeks, patients continued with $6 \mathrm{~g}$ per day of EPA out to 12 weeks. The weight change rate was altered by EPA supplementation from an average loss of $2 \mathrm{~kg} / \mathrm{month}$ before supplementation to a gain of 0.5 $\mathrm{kg} /$ month after four weeks; however, there was no significant change in body composition [33]. A meta-analysis conducted by Colomer et al. found in patients with advanced cancer, a minimum dose of $1.5 \mathrm{~g}$ per day of omega-3 PUFAs is associated with improved weight, appetite, and quality of life [32]. In a double-blind study utilizing a protein and energy-dense supplement enriched with omega3 PUFAs and antioxidants, the supplement resulted in a gain of lean mass and improved quality of life if taken in a high enough dose [14].

The mechanisms through which omega-3 PUFAs are able to stabilize weight in cancer cachexia are not fully understood. However, there is evidence that omega-3 PUFAs might aid in suppressing the acute phase protein response, which is elevated in pancreatic cancer cachexia [35], through the modulation of liver protein export [30]. Omega-3 supplementation may also increase whole-body protein anabolism, as demonstrated by Gingras et al. [36]. Skeletal muscle mTOR and p70S6K1 phosphorylation are increased with EPA and DHA supplementation in young and older adults [37, 38]; however, more work is needed to understand if omega-3 PUFA supplementation can overide 
the mTOR suppression and increased protein degradation in patients with cancer cachexia. Taken together, the results from published data demonstrate potential therapeutic use for omega-3 supplementation in the treatment of cancer cachexia.

\section{Protein}

Cancer patients have abnormal anabolic metabolism of amino acids on muscle protein synthesis [15]. Such Metabolic actions can accelerate muscle loss, which leads to an unfavorable prognosis [39]. Thus, it is essential to care for cancer patients by preventing muscle loss. Protein supplementation is demonstrated to be an essential determinant for muscle maintenance in cancer because optimal protein synthesis is required to take high-quality protein. [40]. On top of that, many studies have shown the different benefits of Protein supplementation, such as elimination of toxins, promotion of tissue repair, destruction of pathogens, and prebiotic effects [41]. Therefore, it is possible to assume that providing enough protein with cancer patients would offer crucial precursors for muscle synthesis and a favorable prognosis by suppressing muscle loss.

Tozer et al. conducted a placebo-controlled double-blind trial over a 6-month to investigate whether a cysteine-rich protein may positively or negatively affect the clinical outcome in non-small cell lung cancer patients [42]. The results report that the patients treated with the cysteine-rich protein displayed the increase of weight and the body cell mass. These results may be associated with an increase in muscle force and specific quality-of-life parameters. The authors suggested that cysteine's metabolic function as a biosynthetic factor of proteins and the cellular anti-oxidant affected an oxidative change in the plasma redox conditions and redox-sensitive signaling pathways in the catabolic process $[43,44]$. This study shows that specific protein supplementation can be part of an anti-cancer cachexia therapy.

Cereda et al. determined the effects of whey protein isolate (WPI) supplementation besides nutritional therapy in malnourished advanced cancer patients during chemotherapy. This study resulted in improved body composition, muscle strength, body weight, and less chemotherapy toxicity [45]. This could be because whey protein includes plenty of leucine and cysteine, a crucial precursor for glutathione synthesis and muscle protein anabolism [46], which could protect cells by decreasing the oxidative stress conditions involved in chemotherapy and preserving anabolic pathways. Additionally, the result is supported by the fact that whey protein supplementation is rich in various essential amino acids, such as leucine, which could affect the results because it is necessary to maintain muscle protein anabolism $[40,47,48]$. This study emphasizes the efficiency of combination with whey protein and standard nutritional care in malnourished patients undergoing anticancer therapy to interrupt a downward flow.

Several studies have used leucine in the context of cachexia with varying results. Some studies show that leucine supplementation attenuated the loss of lean mass and increased protein synthesis, without increasing tumor mass [49-51], while others show no preservation of lean mass with leucine supplementation $[52,53]$. These differences could be related to the timing and dosage of leucine as well as the models used. Despite the differences in the preservation of muscle mass, leucine supplementation has been shown to improve muscle mitochondrial biogenesis and number, and restore tumor-induced losses in energy production [49, 52, 54], which are known to be dysregulated with cancer cachexia and may precede the loss of muscle mass [55]. More work is 
needed to better understand the utility of leucine and protein supplementation as part of a multimodal approach to treating cachexia.

A recent pilot study published by Laviano et al. was conducted to assess an oral targeted medical nutrition supplement's safety and tolerability containing a combination of n-3 PUFAs, 25-hydroxyvitamin D3, and high-quality whey protein in cachexia patients with non-small-cell lung cancer during chemotherapy [56]. This study reported that the targeted medical nutrition, including $\mathrm{n}$ 3PUFAs, Vitamin D3, and whey protein, positively affected several clinical outcomes measures such as gained weight, metabolic markers, and exercise capacity [57]. Given that IGF-1/mTOR, FOXO3a and IL-6 signaling are associated with muscle wasting in cancer cachexia, the therapeutic intervention, including protein supplement, would change the pathways [58]. This study demonstrates that protein supplement is regarded as a vital constitute, and protein can combine with other nutrition supplements to increase treatment efficiency for cachexia patients with cancer.

A recent multimodal study, combining leucine and physical activity, published by Storck et al. conducted a randomized trial in patients with advanced cancer to determine if the addition of leucine-rich whey protein combined with exercise and a standard nutrition program would affect physical performance and quality of life [58]. This study reported that the multimodal program, including a leucine-rich protein, increased handgrip power significantly. Additionally, physical performance and nutritional status exhibited a positive trend even though not statistically significance [58]. The results present meaningful implications because handgrip strength is a significant prognostic predictor associated with mortality, morbidity, and complications [59]. Considering the complex pathophysiology of cachexia, multimodal interventions should be considered. From this study, we can see the feasibility of the combination of protein and exercise to increase treatment efficiency for cachexia patients.

Based on the studies we found out so far, it is reasonable to assume that protein supplement is necessary for cancer patients, considering a potential role of protein on clinical outcomes and part of a multimodal approach for the cachexia patients. Most of the studies we found were conducted with a small sample size. Plus, there are several limitations in a fair comparison, lack of different cases and experiments. Therefore, scientific evidence of protein supplementation would need to be confirmed by large-scale trials and many cases before recommendations to cancer patients with cachexia.

\section{Probiotics and Prebiotics}

The gut microbiota has a crucial effect on biological mechanisms in the human body, including nutrient utilization, resistance against infections, maturation of the immune system, and host metabolism [60,61]. Interestingly, recent studies showed that the balance of gut microbiota is disrupted by tumor cells, cachexia symptoms, and chemotherapy's toxicity [16, 17]. Such a relationship is related to gut permeability, antimicrobial defense, altered intestinal homeostasis, and dysbiosis [62-64]. An unhealthy gut condition would also accelerate the development of a tumor and cancer-related cachexia by fostering harmful bacteria and allowing the translocation of proinflammatory bacterial elements $[16,65]$. We need to look at the gut microbiota as the potential of nutritional therapy with two sides. First is the direct way of affecting gut microbiota metabolism and improving dysbiosis. Second is the indirect way of maximizing cancer-related treatment efficiency by minimizing the side effects of chemotherapy and alleviating cachexia symptoms. 
Bindels et al. conducted an animal experiment to determine if inulin-type fructans (ITF), Lactobacillus strains influence cancer cell proliferation $[66,67]$. This study reported that Inulin-type fructans (ITF) reduce cell infiltration markers, systemic inflammation associated with cancer growth. Moreover, ITF increases portal propionate concentration and the activation of free fatty acid receptors, which would inhibit cancer cell proliferation. They concluded that specific Lactobacillus strains could decrease cancer cell infiltration into the liver and decrease the inflammation [66]. In a subsequent study, the authors investigated the gut microbiota's role in the therapeutic effects on cancer cachexia using two mouse models of cancer cachexia [17]. They found that synbiotic intervention mitigated some of the dysbiotic patterns and restored antimicrobial proteins' expression controlling the intestinal gut barrier and immune system. Such effects led to reduced cancer cell proliferation, muscle wasting and morbidity, and prolonged survival [17]. These studies showed that synbiotics might improve gut microbiota's function that is impaired by cancer-related factors and so be a crucial therapeutic strategy for cancer cachexia.

Cancer patients struggle with cancer treatment because of gastrointestinal symptoms such as abdominal pain, mucositis, diarrhea, and gut toxicity during chemotherapy [68, 69]. These symptoms would interrupt the digestion of nutrients in patients undergoing chemotherapy and cause muscle loss, which eventually leads to the delay of treatment and even can lead to significant morbidity [68, 70,71]. Osterlund et al. conducted randomized clinical trials to assess Lactobacillus's efficacy in reducing chemotherapy toxicity [72]. This study found that Lactobacillus supplementation reduced grade $3 / 4$ diarrhea ( $22 \%$ vs $37 \%$ ) and abdominal discomfort in colorectal cancer patients treated with chemotherapy [74]. In line with the previous study, Motoori et al. (2015) demonstrated that the synbiotics, the combination of probiotics and prebiotics, stabilized intestinal microbiota during and after chemotherapy in advanced esophageal cancer patients, and decreased the frequency of side effects of chemotherapy [73]. The author suggests three mechanisms underlying this data. (1) The change of number in harmful and beneficial bacteria. (2) intestinal motility and concentration of short-chain fatty acid. (3) the improvement of epithelial proliferation and the reduction of intestinal apoptosis [73]. These results showed the practical possibility of synbiotics as supplementary nutrition therapy beyond the theoretical potential.

As the importance of gut microbiota has been emphasized, much research on gut microbiota has been going on. Some studies showed the possibility of probiotics and prebiotics or synbiotics; on the contrary, some studies question the efficacy of the method, and even some studies suggest the risk of gut microbiota as a nutritional intervention [74-76]. For synbiotics to be nutritional intervention, further studies should prove the safety and efficacy in cancer patients.

\section{Vitamin D}

Vitamin D plays a crucial role in bone metabolism and calcium homeostasis (Bouillon and Suda, 2014). In recent years, vitamin D biologic function has been emphasized, including inhibition of cancer cell progression [77]. Vitamin D anti-cancer mechanisms involve inhibition of proliferation, induction of apoptosis and autophagic cell death, and suppressing angiogenesis [78, 79]. Additionally, very recent research confirmed that vitamin D would address immunological infiltrations and cancer stem cell proliferation and affect the tumor microenvironment [80, 81]. With these studies, we can assume that vitamin D has a significant role in tumor suppression. 
Many meta-analysis studies support the assumption by demonstrating an inverse association between vitamin $D$ levels and cancer risk. A review paper with meta-analysis [82] investigated the relationship between cancer risk and vitamin D-related genetic variation and circulating 25hydroxyvitamin D (25OHD) concentration. This study reported that better cancer outcome has to do with higher vitamin $D$ in cancer patients; similarly, low baseline vitamin $D$ is a clear and strong association with worse cancer survival. Under the analysis, a follow-up study in colorectal cancer patients with stage IV found a significant relationship between higher 25-hydroxyvitamin $D$ level and improved survival among patients undergoing chemotherapy [83]. Therefore, given the association between vitamin $D$ deficiency and poor prognosis, vitamin D's nutritional intervention could benefit selected patients.

Akiba et al. conducted a randomized, double-blind trial comparing vitamin D supplements with placebo in advanced non-small cell lung cancer (NSCLC) patients to examine the relapse-free survival and overall survival [84]. This study reported that vitamin D supplements did not improve either relapse-free survival or overall survival of NSCLC patients, including advanced stages and squamous cell carcinoma or large cell carcinoma. Nevertheless, when the analysis was restricted to the subgroup of patients with early adenocarcinoma with lower $25(\mathrm{OH}) \mathrm{D}$, the relapse-free survival and overall survival significantly are improved in the vitamin D group [84]. Similarly, Ng et al. conducted a double-blind phase 2 randomized clinical trial in patients with advanced or metastatic colorectal cancer to determine whether high-dose vitamin D3 improves outcomes during standard chemotherapy [85]. They found that there is not a significant difference in median progression-free survival between the two groups. However, the supportive Cox proportional hazards analysis showed a significantly better hazard ratio in high-dose vitamins than standard-dose vitamins. These studies show that vitamin's effect can differ depending on the degree and type of cancer and the dose of vitamin [85].

Apart from the studies mentioned above, several research pieces have carried out randomized controlled trials to assess the effect of vitamin D supplementation in cancer patients based on rational evidence [86-88]. Part of the data showed positive results in a specific area, and part of the results did not show significant results. In light of it, there are still apparent limitations and considerations to demonstrate the effect of vitamin $D$ in a clinical area such as effect sizes of vitamins does, small study samples, cancer types, and interaction with other nutritional methods. Therefore, further researches have to be warranted to prove vitamin $D$ as nutritional therapy for cancer patients.

\section{Fasting}

While it may seem counterintuitive to utilize fasting to help treat a wasting disease driven by increased catabolic metabolism, there is some evidence that fasting could aid in resetting the metabolic program during cancer cachexia thus attenuating further muscle atrophy. There are several different forms of fasting ranging from time-restricted feeding in which individuals feed for a restricted period and fast the remainder of the day and there can be little impact on caloric intake, to fasting that includes longer durations and has decreased caloric intake. Fasting protocols have many health benefits, including improved glucose tolerance and insulin resistance as well as improved symptoms of hyperlipidemia [89-93]. Both caloric restriction and intermittent fasting are 
associated with reduced cancer incidence [94]. Additionally, fasting is associated with improved cancer treatment efficacy and minimized chemotherapy side effects [95-97].

It has been established that cancer cachexia is associated with altered carbohydrate metabolism, decreased glucose clearance, and lipid dysregulation [98, 99]. While the exact mechanisms of carbohydrate intolerance are not fully understood, a decrease in peripheral glucose uptake and GLUT4 expression are seen in experimental models of cancer cachexia [100-102]. Additionally, cachexia is associated with mitochondrial dysfunction, dysregulation of the ubiquitin proteasomal system, and alteration in autophagy, contributing to muscle loss and metabolic dysfunction [103106]. Intermittent fasting has been shown to improve insulin sensitivity and hyperglycemia in both human and animal models [91, 92, 107]. Fasting increases autophagy, which is already upregulated during cachexia; however, autophagy appears unregulated during cachexia. While the exact mechanisms regulating the disruption in metabolic and mitochondrial function and autophagy are not fully understood, a link between these factors and a loss of circadian function may lead to further explanations.

Changes in circadian rhythm and markers of circadian function have been seen in models of cachexia [108-110], and circadian dysfunction is associated with many of the hallmarks of cachexia, including metabolic dysfunction, dysregulation of autophagic flux, and decreased muscle mass. Harfmann et al. demonstrated that post developmental loss of Bmal1, a regulator of circadian rhythm, in skeletal muscle leads to decreased fat mass, impaired glucose uptake, and insulin resistance [111]. Similar results are seen by others where a loss of muscle Bmal1 leads to the upregulation of lipid metabolism and down-regulation of glucose oxidation [112-114]. Additionally, loss of other clock components leads to decreased skeletal muscle mass and function [115]. Autophagy is also regulated by the circadian clock [116]. Fasting alters clock genes' expression in peripheral tissues such as the liver and muscle [117]. Feeding is a strong regulator of the peripheral clocks and can uncouple the peripheral clocks from the central clock, and the use of fasting and feeding can be used to entrain the peripheral clocks [118-120]. It is currently unknown if fasting and feeding can correct the dysregulation of the peripheral clocks during cancer cachexia. More research is needed to understand better the implications of fasting and fasting mimicking diets on lean mass maintenance and attenuation of metabolic dysfunction during cancer cachexia. Careful monitoring of nutritional status should be utilized in these studies as malnutrition could be a risk.

\section{Ketogenic Diet}

The ketogenic diet is characterized by a low-carbohydrate, high-fat diet that simulates a fasting state without the restriction of calories. When the human body is deprived of carbohydrates, it will naturally shift to producing ketones and fatty acids ( $\beta$ hydroxybutyrate and acetoacetate) as a source of energy [121]. The ketogenic diet has been utilized in pre-clinical and clinical models to target cancer growth [94, 95, 122]. Cancer cells are characterized by the unique phenotype of high aerobic glycolysis rates with lactate production, referred to as the Warburg effect [123]. Thus, cancer cells rely tremendously on glycolysis for ATP to sustain proliferation [124]. The use of the ketogenic diet in cancer patients relies on the fact that cancer cells need glucose for ATP production. However, other tissues in the body, such as the muscle and the brain, can utilize ketone bodies for ATP production and lack of glucose availability for the tumor would prevent further growth [125]. 
Additionally, $\beta$-hydroxybutyrate can suppress the NLRP3 inflammasome, ultimately decreasing IL$1 \beta$ and IL-18 secretion [126].

Several pre-clinical models have shown anti-cancer effects of the ketogenic diet [122]. Additional studies have shown that the ketogenic diet can be utilized to prevent cancer cachexia $[125,127$, 128]. Shukla et al. demonstrated that ketone bodies attenuate both adipocyte and myotube degradation. Additionally, they demonstrate that inhibition of tumor glucose uptake attenuates muscle and fat loss, suggesting that tumor metabolism plays a critical role in the progression of cancer cachexia [125]. Nakamura et al. utilized a colon cancer model and showed that a ketogenic diet decreased tumor mass and preserved muscle mass [128]. They further demonstrated a reduction in plasma IL-6 with the ketogenic diet in tumor-bearing mice [128]. In a clinical study Fearon et al. established that a ketogenic diet could increase body mass; however, the ketogenic diet was unable to alter the nitrogen balance or rates of protein turnover in cachectic patients [129]. Additional research is needed to further understand the ketogenic diet's role in preventing and treating cancer cachexia.

\section{Discussion}

The use of nutritional interventions may provide benefits in the prevention and treatment of cancer cachexia. Individually, omega-3 fatty acids, protein, vitamin D, and probiotics can provide some benefits to cachectic patients through varying mechanisms. Additionally, there are a wide array of other nutritional products that may provide therapeutic value to cachectic patients that were not discussed in this review including curcumin, quercetin, resveratrol, pomegranate, and citrus unshiu peel to name a few. The research to date primarily uses animal models and small sample sizes. More research is needed in clinical populations to further validate the used of these treatment modalities as well as the feasibility of their use. Additionally, studies need to be done accessing the used of these nutritional interventions both as a prevention for cachexia as well as a treatment for cachexia.

Most of the research to date has focused on single supplement or nutritional approached; however, a multimodal approach to treating cancer cachexia should be considered for greater benefits. Multimodal approaches should include more than just a variety of nutritional strategies. Exercise has been shown to prevent and attenuate cachexia progression in animal studies [8], and when combined with protein supplementation is able to improve grip strength in patients with advanced cancer [58]. Additionally, a phase II clinical trial demonstrated an increased body weight with a 12 week multimodal approach combining exercise, anti-inflammatory medications, nutritional counselling, and EPA supplements in patients with lung and pancreatic cancers [130]. This further demonstrates that a multimodal approach is feasible and may provide the most benefit for patients.

There is currently no standard of care treatment for cancer cachexia, a major contributor to cancer-related deaths [1]. The use of dietary interventions shows promise through a variety of mechanisms. Much of the research to date has focused on the prevention and treatment of the tumor without consideration of the presence or impact of cachexia. While much more research is needed, we have demonstrated the potential for the therapeutic use of omega-3 fatty acids, protein, vitamin $D$, probiotics, and fasting and fasting mimicking diets in the treatment and 
prevention of cancer cachexia. The use of nutritional support should be considered as one part of a greater multimodal approach in treating and preventing cancer cachexia.

\section{Author Contributions}

Wangkuk Son conducted a literature review and assisted in writing and editing the manuscript. Jason Lin conducted a literature review and assisted in writing and editing the manuscript. Melissa Puppa conducted a literature review and assisted in writing and editing the manuscript.

\section{Competing Interests}

The authors have declared that no competing interests exist.

\section{References}

1. Tisdale MJ. Mechanisms of cancer cachexia. Physiol Rev. 2009; 89: 381-410.

2. Cespedes Feliciano EM, Lee VS, Prado CM, Meyerhardt JA, Alexeeff S, Kroenke CH, et al. Muscle mass at the time of diagnosis of nonmetastatic colon cancer and early discontinuation of chemotherapy, delays, and dose reductions on adjuvant FOLFOX: The C-SCANS study. Cancer. 2017; 123: 4868-4877.

3. Pin F, Couch ME, Bonetto A. Preservation of muscle mass as a strategy to reduce the toxic effects of cancer chemotherapy on body composition. Curr Opin Support Palliat Care. 2018; 12: 420-426.

4. Dewys WD, Begg C, Lavin PT, Band PR, Bennett JM, Bertino JR, et al. Prognostic effect of weight loss prior to chemotherapy in cancer patients. Am J Med. 1980; 69: 491-497.

5. Ross PJ, Ashley S, Norton A, Priest K, Waters JS, Eisen T, et al. Do patients with weight loss have a worse outcome when undergoing chemotherapy for lung cancers? Br J Cancer. 2004; 90: 1905-1911.

6. Fearon KC, Voss AC, Hustead DS, Cancer Cachexia Study Group. Definition of cancer cachexia: Effect of weight loss, reduced food intake, and systemic inflammation on functional status and prognosis. Am J Clin Nutr. 2006; 83: 1345-1350.

7. Evans WJ, Morley JE, Argiles J, Bales C, Baracos V, Guttridge D, et al. Cachexia: A new definition. Clin Nutr. 2008; 27: 793-799.

8. Puppa MJ, White JP, Velazquez KT, Baltgalvis KA, Sato S, Baynes JW, et al. The effect of exercise on IL-6-induced cachexia in the Apc (Min/+) mouse. J Cachexia Sarcopenia Muscle. 2012; 3: 117137.

9. Prado CM, Sawyer MB, Ghosh S, Lieffers JR, Esfandiari N, Antoun S, et al. Central tenet of cancer cachexia therapy: Do patients with advanced cancer have exploitable anabolic potential? Am J Clin Nutr. 2013; 98: 1012-1019.

10. McGeer AJ, Detsky AS, O'Rourke K. Parenteral nutrition in patients receiving cancer chemotherapy. Ann Intern Med. 1989; 110: 734-736.

11. Klein S, Simes J, Blackburn GL. Total parenteral nutrition and cancer clinical trials. Cancer. 1986; 58:1378-1386. 
12. Bouleuc C, Anota A, Cornet C, Grodard G, Thiery-Vuillemin A, Dubroeucq O, et al. Impact on health-related quality of life of parenteral nutrition for patients with advanced cancer cachexia: Results from a randomized controlled trial. Oncologist. 2020; 25: e843-e851.

13. Gärtner S, Krüger J, Aghdassi AA, Steveling A, Simon P, Lerch MM, et al. Nutrition in pancreatic cancer: A review. Gastrointest Tumors. 2016; 2: 195-202.

14. Fearon KC, Von Meyenfeldt MF, Moses AG, Van Geenen R, Roy A, Gouma DJ, et al. Effect of a protein and energy dense $\mathrm{N}-3$ fatty acid enriched oral supplement on loss of weight and lean tissue in cancer cachexia: A randomised double blind trial. Gut. 2003; 52: 1479-1486.

15. Deutz NE, Safar A, Schutzler S, Memelink R, Ferrando A, Spencer $H$, et al. Muscle protein synthesis in cancer patients can be stimulated with a specially formulated medical food. Clin Nutr. 2011; 30: 759-768.

16. Schwabe RF, Jobin C. The microbiome and cancer. Nat Rev Cancer. 2013; 13: 800-812.

17. Bindels LB, Neyrinck AM, Claus SP, Le Roy Cl, Grangette C, Pot B, et al. Synbiotic approach restores intestinal homeostasis and prolongs survival in leukaemic mice with cachexia. ISME J. 2016; 10: 1456-1470.

18. White JP, Puppa MJ, Gao S, Sato S, Welle SL, Carson JA. Muscle mTORC1 suppression by IL-6 during cancer cachexia: A role for AMPK. Am J Physiol Endocrinol Metab. 2013; 304: E10421052.

19. White JP, Baltgalvis KA, Puppa MJ, Sato S, Baynes JW, Carson JA. Muscle oxidative capacity during IL-6-dependent cancer cachexia. Am J Physiol Regul Integr Comp Physiol. 2011; 300: R201-R211.

20. Giacosa A, Rondanelli M. Fish oil and treatment of cancer cachexia. Genes Nutr. 2008; 3: 25-28.

21. Murphy RA, Mourtzakis M, Chu QS, Baracos VE, Reiman T, Mazurak VC. Nutritional intervention with fish oil provides a benefit over standard of care for weight and skeletal muscle mass in patients with nonsmall cell lung cancer receiving chemotherapy. Cancer. 2011; 117: 1775-1782.

22. Whitehouse AS, Smith HJ, Drake JL, Tisdale MJ. Mechanism of attenuation of skeletal muscle protein catabolism in cancer cachexia by eicosapentaenoic acid. Cancer Res. 2001; 6: 36043609.

23. Smith HJ, Lorite MJ, Tisdale MJ. Effect of a cancer cachectic factor on protein synthesis/degradation in murine C2C12 myoblasts: Modulation by eicosapentaenoic acid. Cancer Res. 1999; 59: 5507-5513.

24. Mitchell T, Clarke L, Goldberg A, Bishop KS. Pancreatic cancer cachexia: The role of nutritional interventions. Healthcare. 2019; 7: 89.

25. Gorjao R, Dos Santos CM, Serdan TD, Diniz VL, Alba-Loureiro TC, Cury-Boaventura MF, et al. New insights on the regulation of cancer cachexia by $\mathrm{N}-3$ polyunsaturated fatty acids. Pharmacol Ther. 2019; 196: 117-134.

26. Lee JY, Sim TB, Lee JE, Na HK. Chemopreventive and chemotherapeutic effects of fish oil derived omega-3 polyunsaturated fatty acids on colon carcinogenesis. Clin Nutr Res. 2017; 6: 147-160.

27. Prado CM, Lieffers JR, McCargar LJ, Reiman T, Sawyer MB, Martin L, et al. Prevalence and clinical implications of sarcopenic obesity in patients with solid tumours of the respiratory and gastrointestinal tracts: A population-based study. Lancet Oncol. 2008; 9: 629-635.

28. Tan BH, Birdsell LA, Martin L, Baracos VE, Fearon KC. Sarcopenia in an overweight or obese patient is an adverse prognostic factor in pancreatic cancer. Clin Cancer Res. 2009; 15: 69736979. 
29. Bruera E, Strasser F, Palmer JL, Willey J, Calder K, Amyotte G, et al. Effect of fish oil on appetite and other symptoms in patients with advanced cancer and anorexia/cachexia: A double-blind, placebo-controlled study. J Clin Oncol. 2003; 21: 129-134.

30. Barber MD, Preston T, McMillan DC, Slater C, Ross JA, Fearon KC. Modulation of the liver export protein synthetic response to feeding by an $n-3$ fatty-acid-enriched nutritional supplement is associated with anabolism in cachectic cancer patients. Clin Sci. 2004; 106: 359-364.

31. Barber MD, Ross JA, Voss AC, Tisdale MJ, Fearon KC. The effect of an oral nutritional supplement enriched with fish oil on weight loss in patients with pancreatic cancer. $\mathrm{Br} J$ Cancer. 1999; 81: 80-86.

32. Colomer R, Moreno-Nogueira JM, García-Luna PP, García-Peris P, García-de-Lorenzo A, Zarazaga A, et al. N-3 Fatty acids, cancer and cachexia: A systematic review of the literature. $\mathrm{Br}$ J Nutr. 2007; 97: 823-831.

33. Wigmore SJ, Barber MD, Ross JA, Tisdale MJ, Fearon KC. Effect of oral eicosapentaenoic acid on weight loss in patients with pancreatic cancer. Nutr Cancer. 2000; 36: 177-184.

34. Murphy RA, Mourtzakis M, Chu QS, Reiman T, Mazurak VC. Skeletal muscle depletion is associated with reduced plasma $(n-3)$ fatty acids in non-small cell lung cancer patients. J Nutr. 2010; 140: 1602-1606.

35. Barber MD, Ross JA, Fearon KC. Changes in nutritional, functional, and inflammatory markers in advanced pancreatic cancer. Nutr Cancer. 1999; 35: 106-110.

36. Gingras AA, White PJ, Chouinard PY, Julien P, Davis TA, Dombrowski L, et al. Long-chain omega3 fatty acids regulate bovine whole-body protein metabolism by promoting muscle insulin signalling to the Akt-mTOR-S6K1 pathway and insulin sensitivity. J Physiol. 2007; 579: 269-284.

37. Smith GI, Atherton P, Reeds DN, Mohammed BS, Rankin D, Rennie MJ, et al. Omega-3 polyunsaturated fatty acids augment the muscle protein anabolic response to hyperinsulinaemia-hyperaminoacidaemia in healthy young and middle-aged men and women. Clin Sci. 2011; 121: 267-278.

38. Smith GI, Atherton P, Reeds DN, Mohammed BS, Rankin D, Rennie MJ, et al. Dietary omega-3 fatty acid supplementation increases the rate of muscle protein synthesis in older adults: A randomized controlled trial. Am J Clin Nutr. 2011; 93: 402-412.

39. Kadar L, Albertsson M, Areberg J, Landberg T, Mattsson S. The prognostic value of body protein in patients with lung cancer. Ann N Y Acad Sci. 2000; 904: 584-591.

40. Devries MC, Phillips SM. Supplemental protein in support of muscle mass and health: Advantage whey. J Food Sci. 2015; 80: A8-A15.

41. Walzem RL, Dillard CJ, German JB. Whey components: Millennia of evolution create functionalities for mammalian nutrition: What we know and what we may be overlooking. Crit Rev Food Sci Nutr. 2002; 42: 353-375.

42. Tozer RG, Tai P, Falconer W, Ducruet T, Karabadjian A, Bounous G, et al. Cysteine-rich protein reverses weight loss in lung cancer patients receiving chemotherapy or radiotherapy. Antioxid Redox Signal. 2008; 10: 395-402.

43. Droge W. Redox regulation in anabolic and catabolic processes. Curr Opin Clin Nutr Metab Care. 2006; 9: 190-195.

44. Hack V, Breitkreutz R, Kinscherf R, Rohrer H, Bartsch P, Taut F, et al. The redox state as a correlate of senescence and wasting and as a target for therapeutic intervention. Blood. 1998; 92: 59-67. 
45. Cereda E, Turri A, Klersy C, Cappello S, Ferrari A, Filippi AR, et al. Whey protein isolate supplementation improves body composition, muscle strength, and treatment tolerance in malnourished advanced cancer patients undergoing chemotherapy. Cancer Med. 2019; 8: 6923-6932.

46. Bounous G, Batist G, Gold P. Whey proteins in cancer prevention. Cancer Lett. 1991; 57: 91-94.

47. Bauer JM, Verlaan S, Bautmans I, Brandt K, Donini LM, Maggio M, et al. Effects of a vitamin D and leucine-enriched whey protein nutritional supplement on measures of sarcopenia in older adults, the provide study: A randomized, double-blind, placebo-controlled trial. J Am Med Dir Assoc. 2015; 16: 740-747.

48. Rondanelli M, Klersy C, Terracol G, Talluri J, Maugeri R, Guido D, et al. Whey protein, amino acids, and vitamin $\mathrm{D}$ supplementation with physical activity increases fat-free mass and strength, functionality, and quality of life and decreases inflammation in sarcopenic elderly. Am J Clin Nutr. 2016; 103: 830-840.

49. Cruz B, Oliveira A, Viana LR, Lopes-Aguiar L, Canevarolo R, Colombera MC, et al. Leucine-rich diet modulates the metabolomic and proteomic profile of skeletal muscle during cancer cachexia. Cancers. 2020; 12: 1880.

50. Eley HL, Russell ST, Tisdale MJ. Effect of branched-chain amino acids on muscle atrophy in cancer cachexia. Biochem J. 2007; 407: 113-120.

51. Gomes-Marcondes MC, Ventrucci G, Toledo MT, Cury L, Cooper JC. A leucine-supplemented diet improved protein content of skeletal muscle in young tumor-bearing rats. Braz J Med Biol Res. 2003; 36: 1589-1594.

52. Lee HW, Baker E, Lee KM, Persinger AM, Hawkins W, Puppa M. Effects of low-dose leucine supplementation on gastrocnemius muscle mitochondrial content and protein turnover in tumor-bearing mice. Appl Physiol Nutr Metab. 2019; 44: 997-1004.

53. Peters SJ, van Helvoort A, Kegler D, Argilès JM, Luiking YC, Laviano A, et al. Dose-dependent effects of leucine supplementation on preservation of muscle mass in cancer cachectic mice. Oncol Rep. 2011; 26: 247-254.

54. Liang C, Curry BJ, Brown PL, Zemel MB. Leucine modulates mitochondrial biogenesis and SIRT1AMPK signaling in C2C12 myotubes. J Nutr Metab. 2014; 2014: 239750.

55. Brown JL, Rosa-Caldwell ME, Lee DE, Blackwell TA, Brown LA, Perry RA, et al. Mitochondrial degeneration precedes the development of muscle atrophy in progression of cancer cachexia in tumour-bearing mice. J Cachexia Sarcopenia Muscle. 2017; 8: 926-938.

56. Laviano A, Calder PC, Schols AM, Lonnqvist F, Bech M, Muscaritoli M. Safety and tolerability of targeted medical nutrition for cachexia in non-small-cell lung cancer: A randomized, doubleblind, controlled pilot trial. Nutr Cancer. 2020; 72: 439-450.

57. White JP, Baynes JW, Welle SL, Kostek MC, Matesic LE, Sato S, et al. The regulation of skeletal muscle protein turnover during the progression of cancer cachexia in the ApcMin/+ mouse. Plos One. 2011; 6: e24650.

58. Storck $\amalg$, Ruehlin M, Gaeumann S, Gisi D, Schmocker M, Meffert PJ, et al. Effect of a leucinerich supplement in combination with nutrition and physical exercise in advanced cancer patients: A randomized controlled intervention trial. Clin Nutr. 2020; 11: S0261-5614(20)301631.

59. Norman K, Stobaus N, Gonzalez MC, Schulzke JD, Pirlich M. Hand grip strength: Outcome predictor and marker of nutritional status. Clin Nutr. 2011; 30: 135-142. 
60. Brestoff JR, Artis D. Commensal bacteria at the interface of host metabolism and the immune system. Nat Immunol. 2013; 14: 676-684.

61. Hooper LV, Littman DR, Macpherson AJ. Interactions between the microbiota and the immune system. Science. 2012; 336: 1268-1273.

62. Lawley TD, Walker AW. Intestinal colonization resistance. Immunology. 2013; 138: 1-11.

63. Lin XB, Dieleman LA, Ketabi A, Bibova I, Sawyer MB, Xue HY, et al. Irinotecan (CPT-11) chemotherapy alters intestinal microbiota in tumour bearing rats. Plos One. 2012; 7: e39764.

64. Puppa MJ, White JP, Sato S, Cairns M, Baynes JW, Carson JA. Gut barrier dysfunction in the $\operatorname{Apc}(\mathrm{Min} /+)$ mouse model of colon cancer cachexia. Biochim Biophys Acta. 2011; 1812: 16011606.

65. Garrett WS. Cancer and the microbiota. Science. 2015; 348: 80-86.

66. Bindels LB, Beck R, Schakman O, Martin JC, De Backer F, Sohet FM, et al. Restoring specific lactobacilli levels decreases inflammation and muscle atrophy markers in an acute leukemia mouse model. Plos One. 2012; 7: e37971.

67. Bindels LB, Neyrinck AM, Salazar N, Taminiau B, Druart C, Muccioli GG, et al. Non digestible oligosaccharides modulate the gut microbiota to control the development of leukemia and associated cachexia in mice. Plos One. 2015; 10: e0131009.

68. Wardill HR, Bowen JM. Chemotherapy-induced mucosal barrier dysfunction: An updated review on the role of intestinal tight junctions. Curr Opin Support Palliat Care. 2013; 7: 155-161.

69. Andreyev J, Ross P, Donnellan C, Lennan E, Leonard P, Waters C, et al. Guidance on the management of diarrhoea during cancer chemotherapy. Lancet Oncol. 2014; 15: E447-E460.

70. van der Meij BS, Deutz NE, Rodriguez RE, Engelen MP. Early signs of impaired gut function in patients with advanced cancer undergoing chemotherapy. J Parenter Enteral Nutr. 2020. DOI: https://doi.org/10.1002/ipen.1941.

71. Antoun S, Raynard B. Muscle protein anabolism in advanced cancer patients: Response to protein and amino acids support, and to physical activity. Ann Oncol. 2018; 29: 10-17.

72. Osterlund $P$, Ruotsalainen $T$, Korpela $R$, Saxelin $M$, Ollus $A$, Valta $P$, et al. Lactobacillus supplementation for diarrhoea related to chemotherapy of colorectal cancer: A randomised study. Br J Cancer. 2007; 97: 1028-1034.

73. Motoori M, Yano M, Miyata H, Sugimura K, Saito T, Omori T, et al. Randomized study of the effect of synbiotics during neoadjuvant chemotherapy on adverse events in esophageal cancer patients. Clin Nutr. 2017; 36: 93-99.

74. Kanazawa H, Nagino M, Kamiya S, Komatsu S, Mayumi T, Takagi K, et al. Synbiotics reduce postoperative infectious complications: A randomized controlled trial in biliary cancer patients undergoing hepatectomy. Langenbecks Arch Surg. 2005; 390: 104-113.

75. McNaught CE, Woodcock NP, Anderson AD, MacFie J. A prospective randomised trial of probiotics in critically ill patients. Clin Nutr. 2005; 24: 211-219.

76. Reid G, Gibson G, Sanders ME, Guarner F, Versalovic J. Probiotic prophylaxis in predicted severe acute pancreatitis. Lancet. 2008; 372: 112-113.

77. Pandolfi F, Franza L, Mandolini C, Conti P. Immune modulation by vitamin D: Special emphasis on its role in prevention and treatment of cancer. Clin Ther. 2017; 39: 884-893.

78. Fleet JC, DeSmet M, Johnson R, Li Y. Vitamin D and cancer: A review of molecular mechanisms. Biochem J. 2012; 441: 61-76. 
79. Umar M, Sastry KS, Chouchane Al. Role of vitamin D beyond the skeletal function: A review of the molecular and clinical studies. Int J Mol Sci. 2018; 19: 1618.

80. So JY, Suh N. Targeting cancer stem cells in solid tumors by vitamin D. J Steroid Biochem Mol Biol. 2015; 148: 79-85.

81. Karkeni E, Morin SO, Tayeh BB, Goubard A, Josselin E, Castellano R, et al. Vitamin D controls tumor growth and CD8+T cell infiltration in breast cancer. Front Immunol. 2019; 10: 1307.

82. Vaughan-Shaw PG, O'Sullivan F, Farrington SM, Theodoratou E, Campbell H, Dunlop MG, et al. The impact of vitamin D pathway genetic variation and circulating 25-hydroxyvitamin $D$ on cancer outcome: Systematic review and meta-analysis. Brit J Cancer. 2017; 116: 1092-1110.

83. Ng K, Sargent DJ, Goldberg RM, Meyerhardt JA, Green EM, Pitot HC, et al. Vitamin D status in patients with stage iv colorectal cancer: Findings from intergroup trial N9741. J Clin Oncol. 2011; 29: 1599-1606.

84. Akiba T, Morikawa T, Odaka M, Nakada T, Kamiya N, Yamashita $M$, et al. Vitamin D supplementation and survival of patients with non-small cell lung cancer: A randomized, double-blind, placebo-controlled Trial. Clin Cancer Res. 2018; 24: 4089-4097.

85. Ng K, Nimeiri HS, McCleary NJ, Abrams TA, Yurgelun MB, Cleary JM, et al. Effect of high-dose vs standard-dose vitamin D-3 supplementation on progression-free survival among patients with advanced or metastatic colorectal cancer the sunshine randomized clinical trial. JAMA. 2019; 321: 1370-1379.

86. Wactawski-Wende J, Kotchen JM, Anderson GL, Assaf AR, Brunner RL, O'Sullivan MJ, et al. Calcium plus vitamin D supplementation and the risk of colorectal cancer. N Engl J Med. 2006; 354: 684-696.

87. Lappe JM, Travers-Gustafson D, Davies KM, Recker RR, Heaney RP. Vitamin D and calcium supplementation reduces cancer risk: Results of a randomized trial. Am J Clin Nutr. 2007; 85: 1586-1591.

88. Urashima $M$, Ohdaira $H$, Akutsu $T$, Okada $S$, Yoshida $M$, Kitajima $M$, et al. Effect of vitamin $D$ supplementation on relapse-free survival among patients with digestive tract cancers the amaterasu randomized clinical trial. JAMA. 2019; 321: 1361-1369.

89. Golbidi S, Daiber A, Korac B, Li H, Essop MF, Laher I. Health benefits of fasting and caloric restriction. Curr Diab Rep. 2017; 17: 123.

90. Hatori M, Vollmers C, Zarrinpar A, DiTacchio L, Bushong EA, Gill S, et al. Time-restricted feeding without reducing caloric intake prevents metabolic diseases in mice fed a high-fat diet. Cell Metab. 2012; 15: 848-860.

91. Brandhorst S, Choi IY, Wei M, Cheng CW, Sedrakyan S, Navarrete G, et al. A periodic diet that mimics fasting promotes multi-system regeneration, enhanced cognitive performance, and healthspan. Cell Metab. 2015; 22: 86-99.

92. Patterson RE, Sears DD. Metabolic effects of intermittent fasting. Annu Rev Nutr. 2017; 37: 371393.

93. Anton SD, Moehl K, Donahoo WT, Marosi K, Lee SA, Mainous AG 3rd, et al. Flipping the metabolic switch: Understanding and applying the health benefits of fasting. Obesity. 2018; 26: 254-268.

94. Lv M, Zhu X, Wang H, Wang F, Guan W. Roles of caloric restriction, ketogenic diet and intermittent fasting during initiation, progression and metastasis of cancer in animal models: $A$ systematic review and meta-analysis. PLoS One. 2014; 9: e115147. 
95. Turbitt WJ, Demark-Wahnefried W, Peterson CM, Norian LA. Targeting glucose metabolism to enhance immunotherapy: Emerging evidence on intermittent fasting and calorie restriction mimetics. Front Immunol. 2019; 10: 1402.

96. de Groot S, Pijl H, van der Hoeven JJ, Kroep JR. Effects of short-term fasting on cancer treatment. J Exp Clin Cancer Res. 2019; 38: 209.

97. Zhang J, Deng Y, Khoo BL. Fasting to enhance cancer treatment in models: The next steps. J Biomed Sci. 2020; 27: 58.

98. Petruzzelli M, Wagner EF. Mechanisms of metabolic dysfunction in cancer-associated cachexia. Genes Dev. 2016; 30: 489-501.

99. Pin F, Barreto R, Couch ME, Bonetto A, O'Connell TM. Cachexia induced by cancer and chemotherapy yield distinct perturbations to energy metabolism. J Cachexia Sarcopenia Muscle. 2019; 10: 140-154.

100.Puppa MJ, Murphy EA, Fayad R, Hand GA, Carson JA. Cachectic skeletal muscle response to a novel bout of low-frequency stimulation. J Appl Physiol. 2014; 116: 1078-1087.

101.Yoshikawa T, Noguchi Y, Satoh S. Inhibition of IRS-1 phosphorylation and the alterations of GLUT4 in isolated adipocytes from cachectic tumor-bearing rats. Biochem Biophys Res Commun. 1999; 256: 676-681.

102.Cersosimo E, Pisters PW, Pesola G, Rogatko A, Vydelingum NA, Bajorunas D, et al. The effect of graded doses of insulin on peripheral glucose uptake and lactate release in cancer cachexia. Surgery. 1991; 109: 459-467.

103.Penna F, Costamagna D, Pin F, Camperi A, Fanzani A, Chiarpotto EM, et al. Autophagic degradation contributes to muscle wasting in cancer cachexia. Am J Pathol. 2013; 182: 13671378.

104.Argiles JM, Lopez-Soriano FJ, Busquets S. Mechanisms to explain wasting of muscle and fat in cancer cachexia. Curr Opin Support Palliat Care. 2007; 1: 293-298.

105.VanderVeen BN, Fix DK, Carson JA. Disrupted skeletal muscle mitochondrial dynamics, mitophagy, and biogenesis during cancer cachexia: A role for inflammation. Oxid Med Cell Longev. 2017; 2017: 3292087.

106. White JP, Baynes JW, Welle SL, Kostek MC, Matesic LE, Sato S, et al. The regulation of skeletal muscle protein turnover during the progression of cancer cachexia in the Apc (Min/+) mouse. PLoS One. 2011; 6: e24650.

107.Lee C, Safdie FM, Raffaghello L, Wei M, Madia F, Parrella E, et al. Reduced levels of IGF-I mediate differential protection of normal and cancer cells in response to fasting and improve chemotherapeutic index. Cancer Res. 2010; 70: 1564-1572.

108.Tsoli M, Schweiger M, Vanniasinghe AS, Painter A, Zechner R, Clarke S, et al. Depletion of white adipose tissue in cancer cachexia syndrome is associated with inflammatory signaling and disrupted circadian regulation. PLoS One. 2014; 9: e92966.

109.Vitale JA, Bonato M, La Torre A, Banfi G. The role of the molecular clock in promoting skeletal muscle growth and protecting against sarcopenia. Int J Mol Sci. 2019; 20: 4318.

110.Counts BR, Hardee JP, Fix DK, Vanderveen BN, Montalvo RN, Carson JA. Cachexia disrupts diurnal regulation of activity, feeding, and muscle mechanistic target of rapamycin complex 1 in mice. Med Sci Sports Exerc. 2020; 52: 577-587. 
111.Harfmann BD, Schroder EA, Kachman MT, Hodge BA, Zhang X, Esser KA. Muscle-specific loss of Bmal1 leads to disrupted tissue glucose metabolism and systemic glucose homeostasis. Skelet Muscle. 2016; 6: 12.

112.Dyar KA, Ciciliot S, Wright LE, Bienso RS, Tagliazucchi GM, Patel VR, et al. Muscle insulin sensitivity and glucose metabolism are controlled by the intrinsic muscle clock. Mol Metab. 2014; 3: 29-41.

113. Hodge BA, Wen Y, Riley LA, Zhang X, England JH, Harfmann BD, et al. The endogenous molecular clock orchestrates the temporal separation of substrate metabolism in skeletal muscle. Skelet Muscle. 2015; 5: 17.

114.Yin H, Li W, Chatterjee S, Xiong X, Saha P, Yechoor V, et al. Metabolic-sensing of the skeletal muscle clock coordinates fuel oxidation. FASEB J. 2020; 34: 6613-6627.

115.Chatterjee $S$, Ma K. Circadian clock regulation of skeletal muscle growth and repair. F1000Res. 2016; 5: 1549.

116. Ma D, Li S, Molusky MM, Lin JD. Circadian autophagy rhythm: A link between clock and metabolism? Trends Endocrinol Metab. 2012; 23: 319-325.

117.Kinouchi K, Magnan C, Ceglia N, Liu Y, Cervantes M, Pastore N, et al. Fasting imparts a switch to alternative daily pathways in liver and muscle. Cell Rep. 2018; 25: 3299-3314 E6.

118.Damiola F, Le Minh N, Preitner N, Kornmann B, Fleury-Olela F, Schibler U. Restricted feeding uncouples circadian oscillators in peripheral tissues from the central pacemaker in the suprachiasmatic nucleus. Genes Dev. 2000; 14: 2950-6291.

119.Stokkan KA, Yamazaki S, Tei H, Sakaki Y, Menaker M. Entrainment of the circadian clock in the liver by feeding. Science. 2001; 291: 490-493.

120.Vollmers C, Gill S, DiTacchio L, Pulivarthy SR, Le HD, Panda S. Time of feeding and the intrinsic circadian clock drive rhythms in hepatic gene expression. Proc Natl Acad Sci. 2009; 106: 2145321458.

121.Longo R, Peri C, Cricri D, Coppi L, Caruso D, Mitro N, et al. Ketogenic diet: A new light shining on old but gold biochemistry. Nutrients. 2019; 11: 2497.

122. Morscher RJ, Aminzadeh-Gohari S, Feichtinger RG, Mayr JA, Lang R, Neureiter D, et al. Inhibition of neuroblastoma tumor growth by ketogenic diet and/or calorie restriction in a CD1-Nu mouse model. PLoS One. 2015; 10: e0129802.

123.Warburg O. On the origin of cancer cells. Science. 1956; 123: 309-314.

124.Poff A, Koutnik AP, Egan KM, Sahebjam S, D'Agostino D, Kumar NB. Targeting the warburg effect for cancer treatment: Ketogenic diets for check for management of glioma. Semin Cancer Biol. 2019; 56: 135-148.

125.Shukla SK, Gebregiworgis T, Purohit V, Chaika NV, Gunda V, Radhakrishnan P, et al. Metabolic reprogramming induced by ketone bodies diminishes pancreatic cancer cachexia. Cancer Metab. 2014; 2: 18.

126.Youm YH, Nguyen KY, Grant RW, Goldberg EL, Bodogai M, Kim D, et al. The ketone metabolite beta-hydroxybutyrate blocks NLRP3 inflammasome-mediated inflammatory disease. Nat Med. 2015; 21: 263-269.

127.Tisdale MJ, Brennan RA, Fearon KC. Reduction of weight-loss and tumor size in a cachexia model by a high-fat diet. Brit J Cancer. 1987; 56: 39-43. 
128. Nakamura K, Tonouchi H, Sasayama A, Ashida K. A ketogenic formula prevents tumor progression and cancer cachexia by attenuating systemic inflammation in colon 26 tumorbearing mice. Nutrients. 2018; 10: 206.

129. Fearon KC, Borland W, Preston T, Tisdale MJ, Shenkin A, Calman KC. Cancer cachexia: Influence of systemic ketosis on substrate levels and nitrogen metabolism. Am J Clin Nutr. 1988; 47: 4248.

130. Solheim TS, Laird BJ, Balstad TR, Stene GB, Bye A, Johns N, et al. A randomized phase II feasibility trial of a multimodal intervention for the management of cachexia in lung and pancreatic cancer. J Cachexia Sarcopenia Muscle. 2017; 8: 778-788.

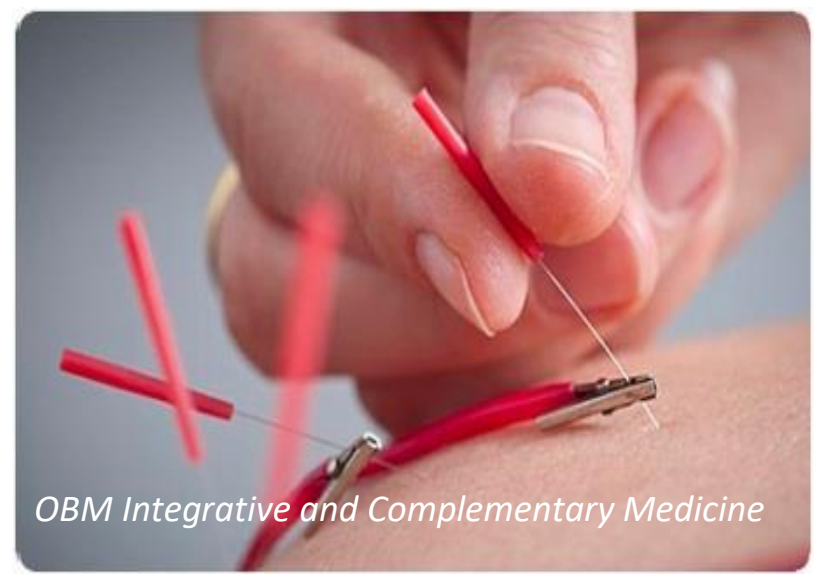

Enjoy OBM Integrative and Complementary Medicine by:

1. Submitting a manuscript

2. Joining in volunteer reviewer bank

3. Joining Editorial Board

4. Guest editing a special issue

For more details, please visit: http://www.lidsen.com/journals/icm 
Research Article

\title{
Auricular Acupuncture with Beads Supports Sustained Weight Loss
}

Takahiro Fujimoto ${ }^{1,2}$, Hidetake Kobayashi ${ }^{3,4}$, Takeshi Hataoka ${ }^{3,4}$, Kazuo Taniguchi ${ }^{4}$, Keisuke Miura 4

1. Clinic F, 1-8-12 Hirakawa-cho, Chiyoda-ku, Tokyo, Japan; E-Mail: fujimoto@clinic-f.com.

2. Tokyo City University Department of Medical Engineering, Faculty of Engineering, 1-28-1 Tamazutumi Setagaya-ku, Tokyo, Japan.

3. Kinki Medical College, 5-3-10 Nishitemma Kita-ku, Osaka, Japan; E-Mails: kobayashi@kinkiisen.ac.jp; t.hataoka@gmail.com.

4. NPO Japan Association of Medical Body Contouring (JAMBC), 2-75-2 Sakuragaoka Yao-shi, Osaka, Japan; E-Mails: sansaido@khf.biglobe.ne.jp; keisuke@holonic.net.

* Correspondence: Takahiro Fujimoto; E-Mail: fujimoto@clinic-f.com.

Academic Editors: Gerhard Litscher and Terry Oleson.

Special Issue: New Trends in Ear Acupuncture

OBM Integrative and Complementary Medicine

2020, volume 5, issue 4

doi:10.21926/obm.icm.2004045
Received: May 17, 2020

Accepted: November 15, 2020

Published: December 09, 2020

\begin{abstract}
Obesity causes serious long-term health problems in people worldwide. Since the effect of dietary advice is possibly limited, weight loss can be a major challenge; therefore, additional weight control techniques may be beneficial. We conducted four experiments to evaluate if auricular acupuncture point stimulation with simple metal beads (AA) rather than the popular use of intradermal needle (DA) to stimulate auricle would support weight loss. In this retrospective study, weight change in Japanese women (aged 18 to 78) was confirmed based on changes in body composition after receiving auricular acupuncture with $A A$ for three months. Furthermore, as a prospective study, we compared three groups-AA, DA, and nonintervention groups - to evaluate if $A A$, which is simpler than DA, can adjust hunger and reduce food intake and snacking $(n=58)$. We evaluated the effect of treatment using a questionnaire that recorded changes in weight, number of snacking time, and the amount of
\end{abstract}

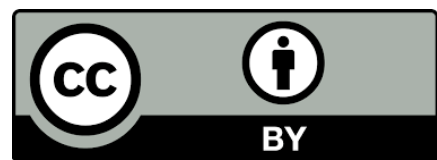


food intake (appetite suppression monitoring study). Furthermore, to evaluate changes in post-prandial blood glucose by AA stimulation by comparing groups, we switched AA and nonintervention periods every $24 \mathrm{~h}$ to measure changes in blood glucose by the AA. Finally, to evaluate long-term weight loss, we conducted a follow-up study 6 months after AA intervention. The rate of weight loss in subjects who received $A A$ intervention ( $n=1362)$ was $-11.15 \%$, confirming a significant decrease. By comparing people who underwent AA with people who did not, we demonstrated that AA reduces snacking between meals and improves satiety. Compared to the non-intervention group (42.75 (65.23) times), the AA (6.6 (10.53) times) and DA (7.93 (4.92) times) groups saw significant decrease in the number of snacking times $(p=0.04$ and $p=0.05)$. The rate of weight loss was $-3.57 \%,-2.74 \%$, and $-1.38 \%$ in the $A A, D A$, and untreated groups, respectively; thus, presenting a significant difference $(p=0.02)$. Glucose decreased in the intervention group, where a significant difference was observed for the median value at 90 and $105 \min (p=0.05, p=0.007$, respectively). Interestingly, weight loss by AA was maintained for six months after the end of treatment. AA had effects equivalent to or even better than DA while being safe and simple. Thus, it can effectively support weight loss and maintain body weight.

\section{Keywords}

Acupuncture; auricular; weight loss

\section{Introduction}

Obesity causes long-term health problems in people with modern lifestyles. Societies are facing an unprecedented increase in obesity, leading to serious problems such as visceral fat, fatty liver, diabetes, cancer, and cardiovascular diseases [1]. Prevention and treatment of obesity-related disorders include a low-calorie diet and regular exercise, but overweight individuals have extremely low compliance with exercise; in many cases, increased appetite caused by exercise cancels any positive impact $[2,3]$.

To solve these problems, we can identify attractive alternative strategies against obesity through acupuncture and alternative medicine, particularly the acupoint stimulation of the auricle. Auricular acupuncture $(A A)$ is said to have existed since the 7th century. It has been considered that people can feel satiated through AA treatment of obesity based on clinical experiences - with a small amount of food, experience less hunger, and see a reduction in food consumption. A simpler method is acupuncture stimulation with beads. In Japan, this method of obesity treatment has over 20 years of history. Previous studies on AA have shown interesting results regarding weight loss in overweight young men [4], young adults [5], obese women [6], and healthy volunteers [7]. AA has been demonstrated to be more effective on weight loss than acupuncture [8]. This method was related to a reduction in the waist circumference of overweight women [9].

Increasing satiety signals reduces food consumption between meals and prevents excess weight gain. Leptin, a peptide hormone produced by adipocytes, is linked to satiety. Its effect on hypothalamic receptors might contribute to obesity by reducing hunger and activating satiety [10]. 
A previous study demonstrated activation of the satiety center by acupoint stimulation of the auricle [11].

Since the effect of dietary advice is limited, the development of acupoint stimulation of the auricle is useful as an effective complementary medicine for people struggling with obesity and overweight [12]. Unlike intradermal acupuncture (DA) treatment, which requires expert acupuncturists and experts of traditional Chinese medicine, our unique method, although not validated, can provide effective dietary support through simple auricular stimulation with beads with a diameter of $1.5 \mathrm{~mm}$. Since it is attached to six points on the ear, no complex expert knowledge or skills are required. In this study, we observed changes in the body composition by a diet method that used beads for stimulation, conducted a follow-up study 6 months later, and examined improvements in the diet and sustainability of weight loss. This method could a more versatile treatment option. We compared hunger, satiety, and reduction in food consumption while comparing the effects of $A A$ and DA with non-intervention. In this manner, we evaluated if the simpler AA could provide an equivalent effect as DD on the ears. Moreover, we measured blood glucose level based on AA and discussed the impact of the auricular acupuncture point stimulation on weight loss.

In our study, we provide effective dietary support through simple auricular stimulation with beads with a diameter of $1.5 \mathrm{~mm}$, although the method has not yet been validated. Since $t$ hese beads are attached to six points on the ear, it does not require complex expert knowledge or skill. To compare if stimulation by beads is as effective as DA as a more versatile treatment method, we added an intradermal group as a control. Moreover, by comparing the reduction in food intake, we examined the sustainability of improved and reduced food intake by auricular stimulation.

\section{Materials and Methods}

\subsection{Auricular Acupuncture (AA) and Intradermal Acupuncture (DA) Point Selection}

We selected 1) ear Shen Men, 2) esophagus, 3) cardia, 4) stomach, 5) lungs, and 6) hypothalamohypophyseal axis for acupoint stimulation of the auricle using $1.5 \mathrm{~mm}$ metallic beads or DA. These points were selected based on the results of previous studies. Figure 1 shows the stimulation points of the auricle.
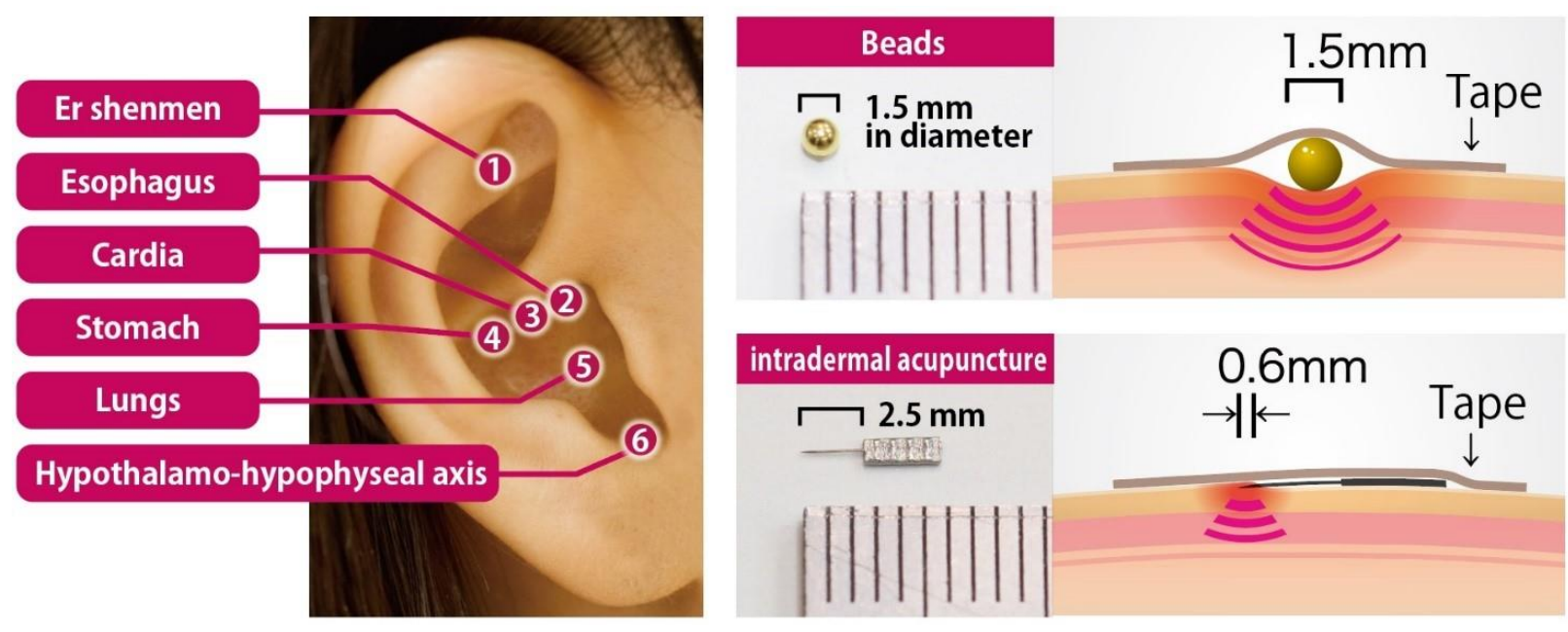

Figure 1 Locations and effects of the auricular acupuncture points. 
1) Ear Shen Men [MA-TF1 Earshenmen] stimulation reduces anxieties and pain and improves mood [13]; thus, presenting the anti-anxiety effect and anti-inflammatory effect [14]. 2) Esophagus and 3) cardia [MA-IC7 Benmen] stimulation regulates appetite and reduces body weight [15] while increasing the sensitivity to salty taste [16]. 4) Esophagus [MA-IC6 Shidao] and stomach [stomach] were selected to stimulate above and below the cardia because the center point can enhance cardiac function. 5) Lung [MA-1C1 Fei] stimulation increases satiety, reduces hunger, and reduces food consumption, leading to weight loss [17] and increasing the sensitivity to salty taste [16]. 6) Hypothalamo-hypophyseal axis [MA-IC3 Neifenmi] stimulation stabilizes the entire endocrine system through the pituitary gland [13]. Codes (MA followed by numbers) are acupuncture points with WHO/WPRO numbers. The use of hunger points has been reported in a previous study on AA to treat overweight people [18]. This point is located on the outside of the ear, and we excluded it, assuming that it would interfere with the subjects' appearance in their daily lives. Anatomically, the afferent nerve is roughly divided into three types: 1 ) the auriculotemporal nerve [the trigeminal nerve ( $C N$ V) the mandibular nerve] dominated region; 2 ) the vagus nerve (X cranial nerve) auricular branch (parasympathetic nerve) dominated region, and 3) the greater occipital nerve (C3 and C4) dominated region [19]. Stimulation in this study was 1) for Ear Shen Men but 2) for other acupuncture points.

The $1.5 \mathrm{~mm}$ metallic beads for the treatment were attached to each acupuncture point of the auricle using a tape. During the measurement, subjects were constantly receiving the stimulus by the beads; however, we had subjects visit the hospital twice a week to replace the beads for hygienic reasons. In terms of stimulation by beads, although seeds could have been used, we used beads created by plating metallic beads for bearing because it allows uniform pressure on each point. Moreover, we could have used magnetic metallic beads; however, we avoided such beads to eliminate magnetism to make a comparison with intradermal needles. Bead replacement for auricular stimulation was performed by treatment staff who had been trained for bead replacement for at least 6 hours using a textbook showing the location of acupuncture points. We assumed that 'the innervation zone' has its impact rather than stimulating traditional 'acupuncture points'; therefore, we organized representative acupuncture points of the lungs into one. Acupuncture point stimulation through acupuncture and moxibustion requires expert knowledge and techniques. We adopted this method based on our clinical experience that a simple method of attaching 1.5-mm beads around acupuncture points suppresses hunger, and offers a wider range of stimulation than acupuncture points due to the distribution of innervation zones.

\subsection{Retrospective Study on Subjects' Weight Change}

This study was a retrospective analysis of data for 1362 Japanese women, between 18 and 78 years of age, who received a 3-month weight-loss program with AA. The data were collected between June 2006 and April 2017 and provided by the NPO Japan Association of Medical Body Contouring (JAMBC). The subjects under 17 years of age were excluded because the calculation method of body composition is different. The subjects had the metallic beads replaced twice a week when visiting the hospital, where they received dietary guidance. Dietary guidance provided at the hospital was different from hospital meals, and managing caloric intake and specifying items was difficult; thus, we used routine meals of each subject before the program participation as the reference. We did not reduce items but instead, we instructed subjects to eat half of each item 
while maintaining a balance. To prevent any nutritional deficit, the subjects were guided in using the nutritional supplement. As a management method for regulation, we had subjects to report their state during hospital visits. We analyzed the rate of weight loss in four groups divided by the degree of obesity and estimated the regression coefficient of the rate of weight loss using multivariate linear regression analysis.

Table 1 Baseline Characteristics of the subjects by BMI.

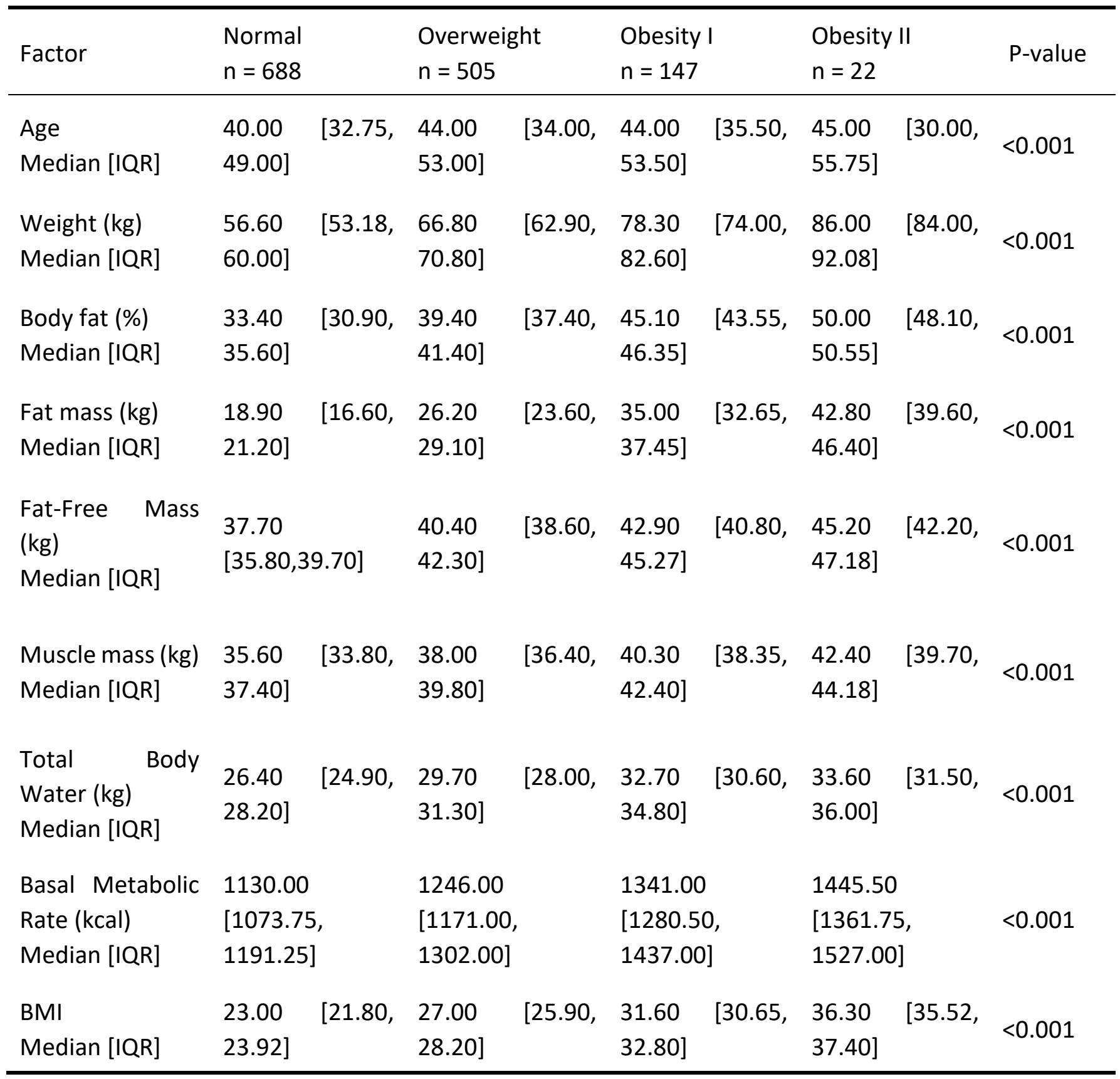

*IQR =Inter Quantile Range

\subsection{Monitoring of Food Consumption}

In this prospective study, we assumed that auricular stimulation suppresses appetite and helps will power. We aimed to demonstrate the impact of auricular stimulation on desire and satiety. 
We recruited student volunteers from Kinki Medical College. The target number of subjects in each group was 23 , and subjects were selected such that age and sex distribution would be as even as possible between the groups. In terms of sex, considering the applicability of the result and ethics of the burden put on by the study, we selected to have half the number of subjects to be male. The subjects were asked to fill out a questionnaire on food consumption, hunger, and satiety during the study period, and the changes in body composition were measured to obtain the objective measurement of the weight reduction effect.

Furthermore, we aimed to examine the difference in the frequency of snacking in the treatment groups with two types of auricular stimulation compared to the no-treatment group. The subjects were then assigned to 1) acupoint stimulation of the auricle by metallic beads (AA), 2) auricular stimulation by intradermal needle (DA), and 3) no-treatment. The caloric intake was reduced to $50 \%$ that of the pre-study level by the same method and was measured for 30 days. No other dietary restriction was imposed. Although double-blind testing is the gold standard, considering the challenges in placebo, we chose to proceed without blinding. In a previous study, multiple efforts were made to create a placebo effect by changing the current velocity and using non-acupuncture points, which did not show desirable results [19]. However, because auricular stimulation with beads is well known, it was assumed that blindness by therapy providers and subjects cannot be assured for AA or DA. With 30 days of reduced food intake, when subjects assigned in the nonintervention group found that their motivation in the study has been lost. It was thought from an ethical viewpoint that it is not appropriate for the subjects in the non-intervention group to undergo reduced food intake, as it might cause physical stress. Therefore, this study was not blinded, as subjects understood that they would be registering to treatment groups. After receiving consent, the assignee randomly assigned the subjects from the applicant list to participate in the study, considering that the ages of each group would be uniform between sexes. These subjects ate three main meals, that is, breakfast, lunch, and dinner per day. All subjects were asked to record the number of times they snacked between meals, satiety, hunger, and the amount of food consumption every $24 \mathrm{~h}$ during the measurement period. Both AA and DA were exchanged twice a week in the treatment group. Body composition was measured at the same timing. The notreatment group was asked to visit the hospital at the same frequency, and their body composition was measured.

Table 2 Questionnaire on eating habits and satiety. This checklist was filled every day by subjects.

\begin{tabular}{lll}
\hline Food intake & Hunger & Satiety \\
\hline $\begin{array}{l}\text { Increased } \\
\text { Unchanged }\end{array}$ & Feel strongly hungry & Feel strongly full \\
$\begin{array}{l}\text { Slightly reduced } \\
\text { Less than } 2 / 3 \text { of the previous } \\
\begin{array}{l}\text { amount } \\
\text { Less than } 1 / 2 \text { of the previous }\end{array}\end{array}$ & Feel full \\
amount & Hardly feel & Feel a little full \\
\hline
\end{tabular}




\subsection{Measurement of Blood Glucose Levels by Auricular Acupuncture}

This prospective study aimed to measure changes in blood glucose level by acupoint stimulation of the auricle. We recruited male and female volunteers of 18-59 years of age at Kinki Medical college. The target sample number was set at 20 . To observe the impact of AA stimulation, we did not provide any specific instruction on the method of dieting regardless of subjects' desire to lose weight. For the same subject, we switched 24-h measurement of intervention and no intervention (recorded at a 15-min interval) every day and took measurement three times daily for six days. No specific restriction was imposed on food consumption or any other lifestyle habits.

For evaluation, in addition to comparing overall measurements, we compared measured values $2 \mathrm{~h}$ after the meal. Since we did not place restrictions, such as standardization of food, it was assumed that there is a notable difference in food consumption, time, and alcohol consumption between breakfast and dinner; thus, we compared the glucose level after lunch, when the dietary habit was relatively stable. In order to compare glucose level when not eating, we compared the data measured when not eating, excluding three main meals and $2 \mathrm{~h}$ after these three meals between AA and no intervention. Since it was a comparison of the same person, and the subject was aware of intervention based on the presence of beads, the comparison could not be blinded. Rather, AA and no intervention were switched every day, and evaluation was made based on repetitive data. For measurement, a flash glucose monitoring system (FreeStyle Libre, Abbott: flash glucose monitoring measurement range of $40-500 \mathrm{mg} / \mathrm{dL}$, the accuracy of $\pm 15 \mathrm{mg} / \mathrm{dL}( \pm 0.83$ $\mathrm{mmol} / \mathrm{L}$ ), and $\pm 20 \%$ of the reference value) was used.

\subsection{Analysis of Weight and Body Composition}

To examine changes in weight after the acupuncture point stimulation, we recruited volunteers from-the retrospective study on subjects' weight change (subjects who had the treatment less than six months or longer than six months were excluded). In order to measure body weight and composition, we used dual-frequency body composition analyzer DC-430-A (TANITA Corporation).

The measurement method was the dual-frequency BIA method where the weak high-frequency alternating current was applied, and body composition was calculated based on the electric resistance. Measurement frequencies were $6.25 \mathrm{kHz} / 50 \mathrm{kHz}$, and the current was $90 \mu \mathrm{A}$ or below.

\subsection{Statistical Analysis}

The R software version 3.6.0 was used for data analysis. In the retrospective study on subjects' weight change under Section 2.2, repeated-measures ANOVA was performed. Then, Paired t-test and multivariate linear regression analysis were performed.

For the three-group trial described in Section 2.3, we performed the Kruskal-Wallis rank-sum test and then used the Welch two-sample t-test to avoid problems associated with multiple tests for body composition measurements. For the questionnaire data analysis, we used chi-square test.

In the blood glucose tests under Section 2.4, we performed the Friedman rank-sum test and then used the Wilcoxon signed-rank test for intergroup comparison.

A paired $t$-test was used for the rebound test described in Section 2.5. Significance level was $P<$ 0.05 .

This study only used existing anonymized data that cannot be linked. 


\section{Results}

\subsection{Changes in Body Composition by AA}

The data collected from the weight loss program by auricular acupuncture ( $n=1362$ ) had an overall rate of weight loss of $-11.15 \%$ (5.73). Moreover, we performed an analysis in groups separated by the obesity level based on BMI and reported that the normal group $(n=688)$, overweight group ( $n=505)$, obese I group $(n=147)$, and obese II group $(n=22)$ had a rate of weight loss of $-10.10 \%$ (5.65), $-12.04 \%$ (5.59), $-13.01 \%$ (5.61), and $-10.95 \%$ (5.64), respectively. The regression coefficient of the rate of weight loss demonstrated that each time BMI increased by 1 , a $-0.2 \%$ weight loss occurred with a significant difference.

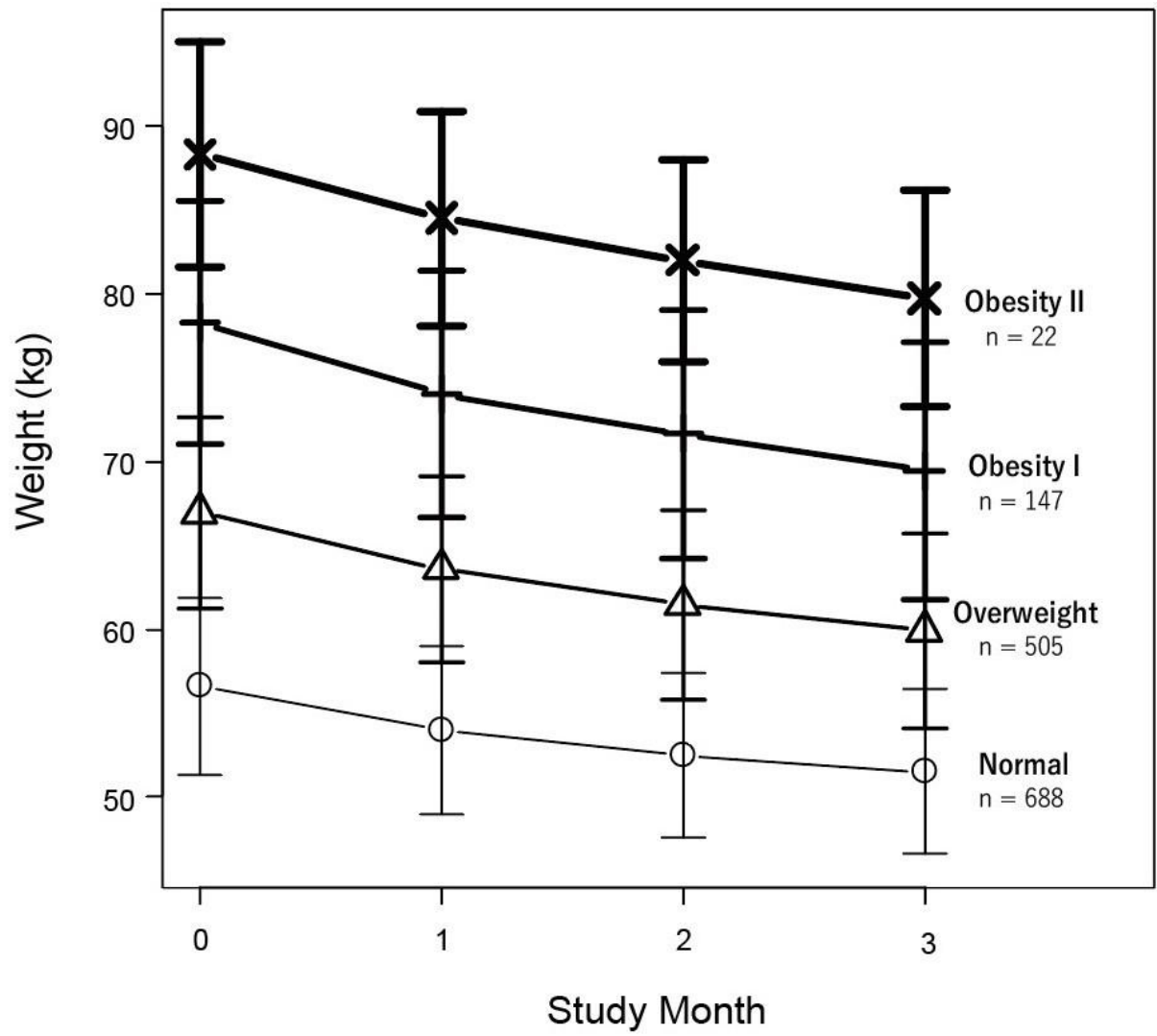

Figure $\mathbf{2}$ Changes in body weight in groups separated by the degree of obesity based on BMI over three months.

Significant difference in repeated measures ANOVA $(P<0.001)$.

Table 3 Age, baseline body weight, and estimated rate of weight loss adjusted to BMI.

Variable $\quad$ Estimate $(95 \% \mathrm{Cl}) \quad$ P-value
Age
$0.02(0-0.05)$
0.079 


\begin{tabular}{lll} 
BMI & $-0.24(-0.42--0.05)$ & 0.012 \\
Weight $(\mathrm{kg})$ & $-0.05(-0.11-0.02)$ & 0.19 \\
\hline
\end{tabular}

\subsection{Comparison of AA and DA on Body Weight Loss}

After the recruitment, 58 men and women of 21-60 years of age participated in the study (23 in the AA group, 17 in the DA group, and 18 in the no-treatment group).

Table 4 Patients' Baseline Characteristics in three-group comparison trial for the AA group, DD group, and no-treatment group.

\begin{tabular}{|c|c|c|c|c|c|c|c|c|}
\hline Factor & Group & $\begin{array}{l}\text { AA } \\
n=23\end{array}$ & & $\begin{array}{l}D A \\
n=17\end{array}$ & & $\begin{array}{l}\text { No trea } \\
\mathrm{n}=18\end{array}$ & ment & P-value \\
\hline Sex (\%) & Female & $11(47$ & & 9 (52.9) & & \multirow{2}{*}{\multicolumn{2}{|c|}{$\begin{array}{l}9(50.0) \\
9(50.0)\end{array}$}} & \multirow{2}{*}{0.95} \\
\hline No.(\%) & Male & \multicolumn{2}{|c|}{$\begin{array}{l}11(4 / .8) \\
12(52.2)\end{array}$} & $8(47.1)$ & & & & \\
\hline Age & & 39.00 & \multirow[t]{2}{*}{34.00,} & 36.00 & \multirow[t]{2}{*}{26.00,} & 41.00 & {$[35.25$,} & \multirow{2}{*}{0.108} \\
\hline Median [IQR] & & 47.00] & & 42.00] & & 46.50] & & \\
\hline BMI & & 27.20 & \multirow[t]{2}{*}{24.75,} & 26.70 & \multirow[t]{2}{*}{25.20,} & 23.25 & {$[20.88$,} & \multirow{2}{*}{0.001} \\
\hline Median [IQR] & & 31.50] & & 29.00] & & 24.98] & & \\
\hline Body weight (kg) & & 71.60 & \multirow[t]{2}{*}{64.20,} & 76.10 & \multirow[t]{2}{*}{56.10,} & 64.80 & {$[58.52$,} & \multirow{2}{*}{0.049} \\
\hline Median [IQR] & & 92.75] & & $80.00]$ & & 67.83] & & \\
\hline
\end{tabular}

*IQR = Inter Quantile Range

We conducted the Kruskal-Wallis rank-sum test on the AA group, DA group, and nonintervention group and reported a significant difference in the rate of weight loss $(P=0.02)$. Thus, we compared the baseline and the final weight of each group through the paired t-test. The result demonstrated that the weight decrease for AA, DA, and no-treatment groups was $78.81 \mathrm{~kg}(18.08)$ $\rightarrow 76.10 \mathrm{~kg}$ (17.18), $71.36 \mathrm{~kg}(14.95) \rightarrow 70.91 \mathrm{~kg}$ (14.58), and $64.7 \mathrm{~kg}(0.12) \rightarrow 62.59 \mathrm{~kg}(11.48)$, respectively; thus, presenting significant difference in each group $(P<0.001, P<0.001$, and $P=0.05$, respectively).

When we compared to the AA group and non-intervention group, a significant decrease in body weight was observed in the AA group with $-3.39 \%$ (2.26) and the non-intervention group with $1.52 \%$ (1.71) $(P=0.004)$. The difference between the DA group and non-intervention group was $2.34 \%$ (1.79), which was not statistically significant $(P=0.18)$. There was no significant difference between the AA group and the DA group $(P=0.11)$. 


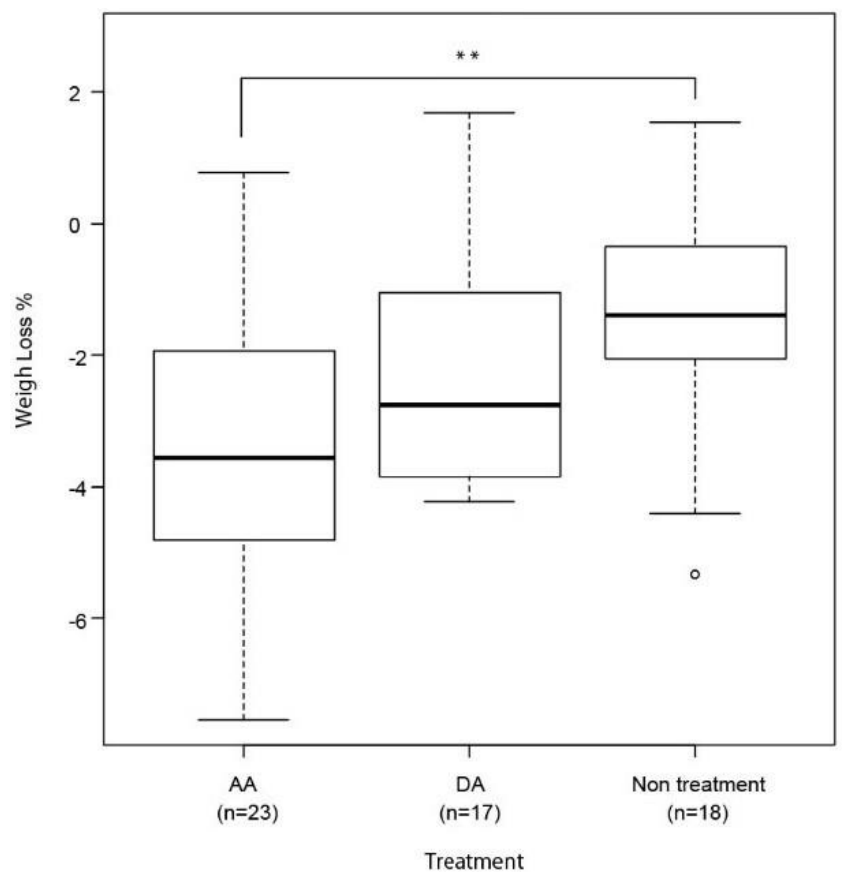

Figure 3 Median of weight loss in each treatment group after 30 days, expressed in percentage of the initial weight. ${ }^{* *}=\mathrm{P}<0.01,{ }^{\circ}=$ outliers.

In order to compare the number of snacking during food consumption reduction over 30 days, certain subjects did not respond to necessary questions on the questionnaire. Therefore, a comparison was made between 23 subjects in the AA group, 14 subjects in the DA group, and 16 subjects in the no-treatment group.

Since the Kruskal-Wallis rank-sum test confirmed a significant difference $(P=0.001)$, we compared with the non-intervention group 42.75 (65.23 times) and reported that it was 6.64 (10.54 times) $(P=0.04)$ and 7.93 (4.92 times) $(P=0.05)$ for the $A A$ and $D A$ groups, respectively.

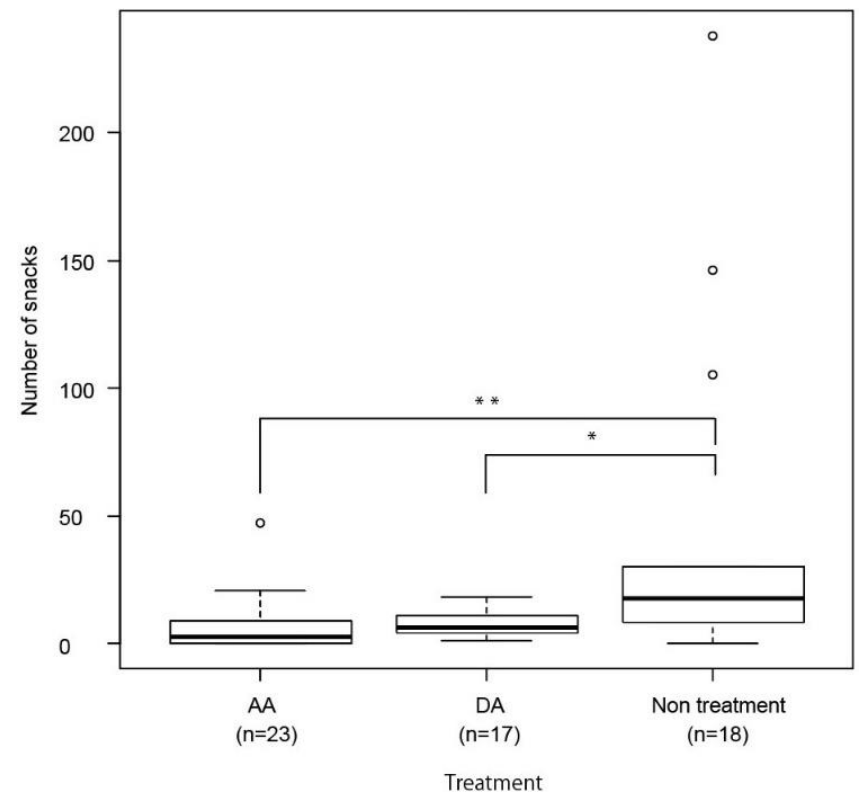

Figure 4 Intergroup comparison of the number of snacks. ${ }^{*}=\mathrm{P}<0.05,{ }^{* *}=\mathrm{P}<0.01,^{\circ}=$ outliers. 


\subsection{Effects of $A A, D A$, and no Treatment on Satiety}

It was observed in the questionnaire that one person missed answers in both DA and notreatment groups. Therefore, the analysis was performed with 23 subjects in the AA group, 16 subjects in the DA group, and 17 subjects in the no-treatment group. After 30 days of treatment, those who answered with 'able to reduce in half": 'unchanged' + 'increased' regarding the amount of food consumed during the measurement period were $51 \%: 7 \%$ for the AA group, $33.1 \%: 16 \%$ for the DA group, and 18.4\%:29\% for the no-treatment group. In terms of hunger, those who answered 'hardly felt hungry': 'strongly felt hunger' were $45.6 \%: 7 \%$ for the AA group, $36.5 \%: 8.5 \%$ for the DA group, and $20.7 \%: 26.5 \%$ for the no-treatment group. For satiety, those who answered 'did not feel full': 'felt strong fullness' were 5.5\%:11.7\% for the AA group and 16.6\%:7.7\% for the DA group, which does not confirm independence in any item $(P<0.001)$.

Table 5 Questionnaire response regarding food amount.

\begin{tabular}{|c|c|c|c|c|c|}
\hline Group & $\begin{array}{l}\text { Less than } 1 / 2 \text { of } \\
\text { the previous } \\
\text { amount }\end{array}$ & $\begin{array}{l}\text { Less than } \\
2 / 3 \text { of the } \\
\text { previous } \\
\text { amount }\end{array}$ & $\begin{array}{l}\text { Slightly } \\
\text { reduced }\end{array}$ & Unchanged & Increased \\
\hline $\begin{array}{l}\text { AA } \\
\text { Frequencyr } \\
\text { (\%) dij }\end{array}$ & $\begin{array}{l}342(51.5) 14.3 \\
* *\end{array}$ & $\begin{array}{lll}146 & (22)- \\
2.1 & & \\
* & & \end{array}$ & $\begin{array}{ll}130 & (19.6)- \\
6.4 & \\
* * & \end{array}$ & $\begin{array}{l}37(5.6)-18.2 \\
* *\end{array}$ & $\begin{array}{l}9(1.4)-22.4 \\
* *\end{array}$ \\
\hline $\begin{array}{l}\text { DA } \\
\text { Frequencyr } \\
\text { (\%) dij }\end{array}$ & $\begin{array}{l}155(33.1)-2.2 \\
*\end{array}$ & $\begin{array}{l}152 \quad(32.5) \\
9.9 \\
* *\end{array}$ & $\begin{array}{l}86(18.4)-6.2 \\
* *\end{array}$ & $57(12.2) 0.1$ & $18(3.8)-1.4$ \\
\hline $\begin{array}{l}\text { No treatment } \\
\text { Frequencyr } \\
\text { (\%) dij }\end{array}$ & $\begin{array}{l}91(18.4)-13.1 \\
* *\end{array}$ & $\begin{array}{l}83(16.8)- \\
7.6 \\
* *\end{array}$ & $\begin{array}{l}177 \\
12.9 \\
* *\end{array}$ & $\begin{array}{l}104 \quad(21.1) \\
19.5 \\
* *\end{array}$ & $\begin{array}{l}39(7.9) 25.2 \\
* *\end{array}$ \\
\hline
\end{tabular}

dij $=$ Adjusted residual, ${ }^{*} \mathrm{P}<0.05,{ }^{* *} \mathrm{P}<0.01$.

Table 6 Questionnaire responses on the sensation of hunger.

\begin{tabular}{llll}
\hline Group & Hardly feel & Feel hungry & Feel strongly \\
\hline $\begin{array}{l}\text { AA } \\
\text { frequencyr (\%) dij }\end{array}$ & $\begin{array}{l}308(45.6) 9.6 \\
* *\end{array}$ & $\begin{array}{l}321(47.5)-2.5 \\
*\end{array}$ & $\begin{array}{l}47(7)-16.2 \\
* *\end{array}$ \\
DA & & $258(55) 1.9$ & $\begin{array}{l}40(8.5)-9.1 \\
* *\end{array}$ \\
frequencyr (\%) dij & $171(36.5) 0.7$ & & $128(26.5) 26.4$ \\
No treatment & $100(20.7)-11$ & $255(52.8) 0.9$ & $* *$ \\
frequencyr (\%) dij & $* *$ & & \\
\hline
\end{tabular}

dij $=$ Adjusted residual, $* \mathrm{P}<0.05,{ }^{* *} \mathrm{P}<0.01$. 
Table 7 Questionnaire responses on the sensation of satiety.

\begin{tabular}{|c|c|c|c|c|}
\hline Group & Do not feel full & Feel a little full & Feel full & Feel strongly full \\
\hline $\begin{array}{l}\text { AA } \\
\text { frequencyr (\%) dij }\end{array}$ & $\begin{array}{l}37(5.5)-20.6 \\
* *\end{array}$ & $263(39.3) 1.9$ & $\begin{array}{l}291(43.5) 2.5 \\
*\end{array}$ & $\begin{array}{l}78(11.7) 15.2 \\
* *\end{array}$ \\
\hline $\begin{array}{l}\text { DA } \\
\text { frequencyr (\%) dij }\end{array}$ & $\begin{array}{l}75(16.6) 4.5 \\
* *\end{array}$ & $172(38) 0.5$ & $171(37.7)-1.8$ & $35(7.7)-1.1$ \\
\hline $\begin{array}{l}\text { No treatment } \\
\text { frequencyr (\%) dij }\end{array}$ & $\begin{array}{l}113(23.5) 17.8 \\
* *\end{array}$ & $\begin{array}{l}163(34)-2.4 \\
*\end{array}$ & $188(39.2)-1$ & $\begin{array}{l}16(3.3)-15.4 \\
* *\end{array}$ \\
\hline
\end{tabular}

$$
\text { dij }=\text { Adjusted residual, } * \mathrm{P}<0.05, * * \mathrm{P}<0.01
$$

\subsection{Effects of $A A$ on post-prandial glucose levels}

In total, 13 men and women who were 19-52 years old participated in the study. Among subjects, two showed abnormalities in the measurement device; thus, the analysis was performed with 13 subjects.

Table 8 Patients Baseline Characteristics in a follow-up survey six months after the end of the treatment.

\begin{tabular}{ll}
\hline Factor & $\begin{array}{l}\text { Overall } \\
\mathrm{n}=13\end{array}$ \\
\hline $\begin{array}{l}\text { Sex } \\
\text { No. (\%) }\end{array}$ & $\begin{array}{l}\text { Female } 3(23.1) \\
\text { Male } 10(76.9)\end{array}$ \\
$\begin{array}{l}\text { Age } \\
\text { Median [IOR] }\end{array}$ & $27.00[24.00,36.00]$ \\
BMI & \\
Median [IOR] & $22.60[20.60,25.30]$ \\
Weight (kg) & \\
Median [IQR] & $60.00[59.30,77.10]$ \\
\hline
\end{tabular}

Median [IQR], *IQR = Inter Quantile Range 


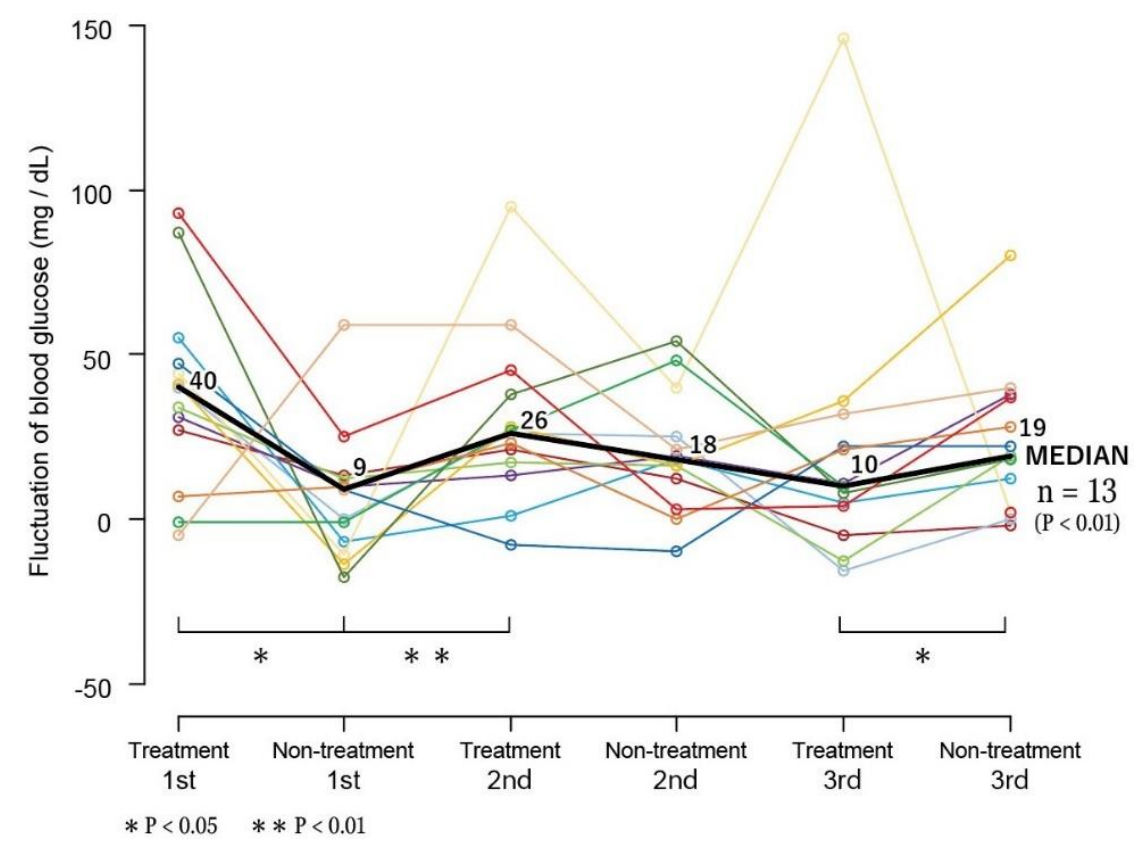

Figure 5 Changes in post-prandial blood glucose by the bead treatment (after $105 \mathrm{~min}$ ).

The glucose level for non-intervention and intervention groups was $100.62 \mathrm{mg} / \mathrm{dL}$; however, it was $98.48 \mathrm{mg} / \mathrm{dL}$ for the intervention group. We compared the post-prandial changes for every 15 min till $2 \mathrm{~h}$ after lunch based on the treatment and confirmed a significant difference for $90 \mathrm{~min}$ and $105 \mathrm{~min}(\mathrm{P}=0.05$ and $\mathrm{P}=0.007$, respectively). Then, we examined the changes at $105 \mathrm{~min}$ and reported that it was $+40 \rightarrow+9 \mathrm{mg} / \mathrm{dL}$ after the first treatment, $+9 \rightarrow+26 \mathrm{mg} / \mathrm{dL}$ after the second non-treatment, and $+10 \rightarrow+19 \mathrm{mg} / \mathrm{dL}$ after the third non-treatment; thus, confirming significant differences $(P=0.03,0.01$, and 0.04 , respectively). Moreover, in a comparison that excluded data from $2 \mathrm{~h}$ from the start breakfast, lunch, and dinner, i.e., non-meal times, blood glucose was 96.55 $\mathrm{mg} / \mathrm{dL}$ for the non-intervention group and $94.75 \mathrm{mg} / \mathrm{dL}$ for the intervention group where the latter had a significantly lower value $(P<0.001)$.

\subsection{Weight and Body Composition Six Months Post Treatment}

Among the 1,362 subjects of the retrospective study on subjects' weight change, we were able to obtain measurement data from 57 subjects after six months.

Table 9 Baseline Characteristics of the Patients.

\begin{tabular}{ll}
\hline Factor & $\begin{array}{l}\text { Overall } \\
\mathrm{n}=57\end{array}$ \\
\hline Sex (\%) & $57(100.0)$ \\
Age & $48.00[39.75,53.75]$ \\
Median [IOR] & $24.60[23.40,26.70]$ \\
BMI & \\
Median [IOR] & \\
\hline
\end{tabular}




$$
\begin{array}{lc}
\text { Weight }(\mathrm{kg}) & 62.20[57.10,67.50] \\
\text { Median [IQR] } &
\end{array}
$$

Median [IQR], *IQR = Inter Quantile Range.

The result demonstrated that no significant difference was present in body fat percentage, basal metabolic rate, muscle mass, and fat mass between the end of the study and six months after the treatment where the weight loss effect was maintained six months later, and reduction in both weight $(-0.8)$ and BMI $(-0.3)$ was significant. The change in the body weight six months after the treatment was $-1.3 \pm 4.2 \mathrm{~kg}(\mathrm{P}<0.01)$; however, the rate of weight change between the baseline and six months after the end of the treatment was $-11.1 \% \pm 8.8 \%(P<0.01)$.
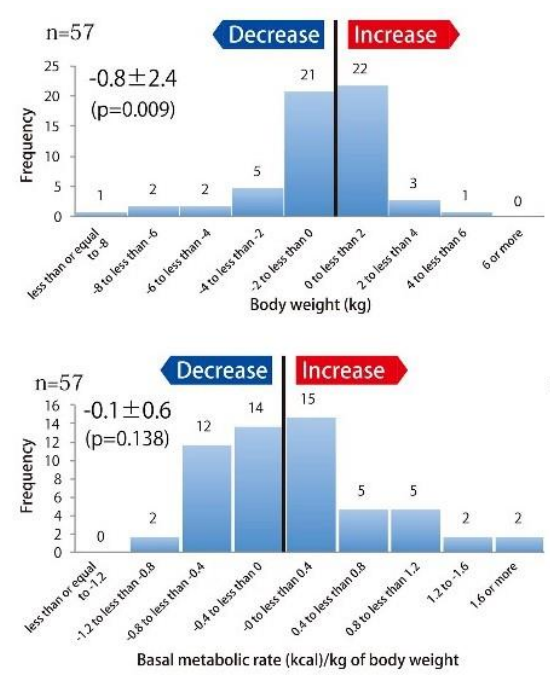
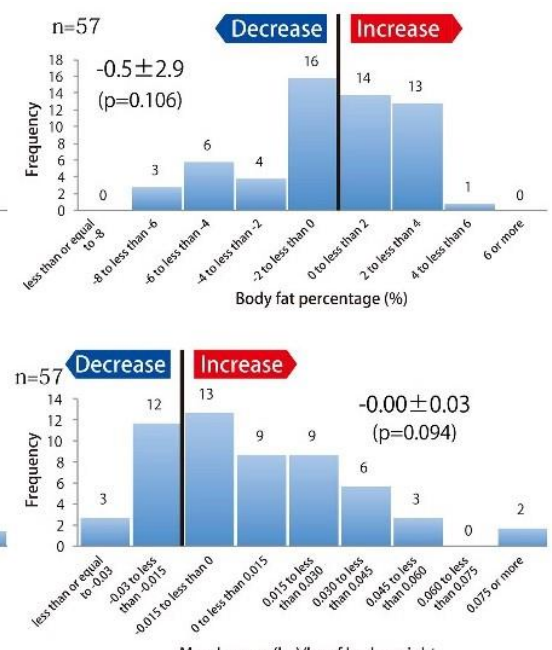

Muscle mass $(\mathrm{kg}) / \mathrm{kg}$ of body weight

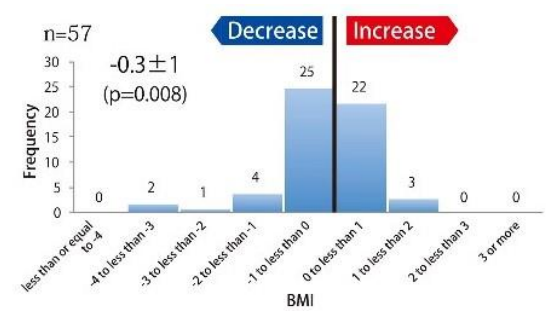

BMI

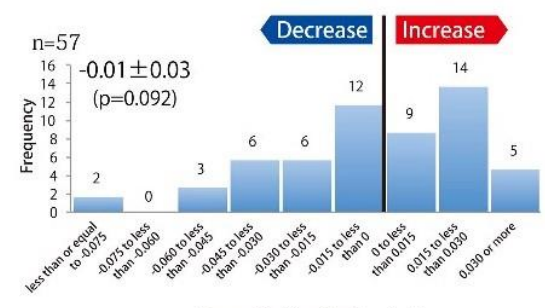

Fat mass $(\mathrm{kg}) / \mathrm{kg}$ of body weight

Figure 6 Frequency distribution of changes in body weight and body compositions six months after the treatment.

Figure 6 shows the change in the body composition of subjects $(n=57)$ from the end of the treatment to six months after the end of the treatment as frequency distribution. The boundary between reduction and increase is shown with a solid line. Each measured item was analyzed with the paired t-test.

\section{Discussion}

Interestingly, in the retrospective study of weight change, the subjects were mainly women. This can be explained as it is an observational study and most subjects who preferred to diet were women.

The goal for weight loss programs is usually $-10 \%$ (average $-8 \%$ ) of the body weight in six months [20]. In a study that summarized acupuncture to manage obesity [21], compared to sham needling, there was a weight loss of $-1.54 \mathrm{~kg}$; moreover, compared to diet and exercise, there was a weight loss of $-3.27 \mathrm{~kg}$. However, the data of the weight loss program by AA that we collected ( $\mathrm{n}=1362$ ) showed an overall weight loss of $-11.15 \%$ (5.73) in three months. For the obesity I group, a weight loss of $-13.01 \%$ (5.61) occurred, which was notably higher than the average.

However, data used in this study are taken from an observation of a single group, and reduction in food intake was a direct result of being asked to reduce food consumption by half; thus, there 
was no quantitative observation of food intake reduction by will power. Performing repeat analysis, such as crossover test, incorporating a research design that evaluates changes between individuals, and observing long-term changes would be effective in solving this problem.

In the appetite suppression monitoring study, AA demonstrated the most weight loss rate and the least frequency snacking between meals. Beads have a wider range of stimulation than intradermal needles. Unlike leaving intradermal needles in situ, when those in the AA group felt stress from hunger, additional stimulation was applied, such as application of pressure at the treatment sites. Thus, these additional stimulations and psychological peace might amplify the amount of stimulation. However, with an intradermal needle, no additional stimulation is caused, as ears have few voluntary muscles, unlike joints. The stimulation effect might decrease as the subject becomes accustomed to stimulation. Even without acupuncture and moxibustion stimulation that requires a special technique, AA can sufficiently reduce hunger, among other changes. By comparing the snacking tendency and hunger when the food consumption was reduced to half in this study, we confirmed that there is a conscious reduction in food consumption. The use of false acupoints with the innervation zone in the control group would be questionable because it may cause adverse effects. Moreover, in this trial, we concluded that there would be a reduction effect on hunger; however, because psychological elements of subjects who strongly wish for weight loss have a notable impact on the result, for non-blind trial or when blindness is broken, weight reduction effect might drastically decrease, and reduced motivation might have a notable impact on the result. Since the study was not blind, the challenge is removing the bias by making the acupuncture treatments such as AA and DA blind and knowing the difference in the methods.

The result of the study to measure changes in blood glucose by AA confirmed that AA suppresses an increase in blood glucose. This suppression effect was most prominent between 90 and 105 min after the start of a meal. The suppression effect on blood glucose was clear at the non-meal time as well, indicating that AA delays increase in blood glucose and potentially suppresses insulin secretion.

Since it is well known that the satiety center is located in the ventromedial hypothalamus and the feeding center is located outside the hypothalamus, we assumed that AA reduces appetite through the medial hypothalamus [17]. A previous study on obese rats showed that electric stimulation of the low resistance region of the ear reached the hypothalamus through the auricular branch of the vagus nerve, stimulating neurons associated with feeding adjustment. Rats that are not very obese experienced the promotion of leptin production in peripheral white adipose tissue, and binding with leptin receptors (Ob-R) in the peripheral and central nervous system regulates feeding [22]. In this study, we proposed that the stimulation of the auricular acupoints with beads leads to the expression of leptins from adipocytes to the vagus nerve. We attempted to make repetitive measurements using the crossover test method because of the difficulty of blinding AA; however, by having a longer duration of measurement for each method, mode detailed data can be obtained.

The retrospective study on subjects' weight change confirmed the result of a previous study on the impact of acupuncture on the ear on body weight [23]. Furthermore, using the follow-up study, six months after the AA intervention, we clearly showed a statistically significant difference in subjects whose weight continued to drop even after the treatment had ended. This may be caused by improved body type because of change in diet and resetting of the point comprising leptin sensitivity at the satiety center as a result of AA. However (though this is true for all studies), subjects are all Japanese women, and a study on multiple races would be desired. 
In conclusion, AA might offer a method of weight reduction, in addition to diet and exercise. We demonstrated that even a simple method of adhering beads to the auricle can offer an effect equivalent or better than intradermal needles that require a high level of knowledge and technique. AA has been demonstrated to reduce hunger caused by dietary restrictions to lose weight and help consciously control food consumption.

From the perspective of weight loss maintenance upon the end of the program, AA might improve lifestyle-related diseases.

\section{Acknowledgments}

The authors are grateful to Akira Suzuki, Hiroshi Osawa, Akihiko Kobayashi, Katsushi Kitagawa, Keiichi Asami, Kenichi Koizumi, Machiko Kigoshi, Masahiro Yamada, and Nichihide Maki, board members of Japan Association of Medical Body Contouring (JAMBC), for his encouragement in the performance of this study.

\section{Author Contributions}

Takahiro Fujimoto conceived and planned the experiments. Hidetake Kobayashi and Takeshi Hataoka planned and supervised all the experiments. Takeshi Hataoka also produced formats for all the experiments and was responsible for their distribution, collection, data input for tabulation, and preparation of figures and tables. Takahiro Fujimoto and Takeshi Hataoka were responsible for statistical analysis. Takahiro Fujimoto took the lead in writing the manuscript. Hidetake Kobayashi, Kazuo Taniguchi, and Keisuke Miura contributed to the interpretation of the results. All authors provided critical feedback and helped shape the research, analysis, and manuscript.

\section{Competing Interests}

The authors have declared that no competing interests exist.

\section{References}

1. Eckel RH, Grundy SM, Zimmet PZ. The metabolic syndrome. Lancet. 2005; 365: 1415-1428.

2. Kraschnewski JL, Boan J, Esposito J, Sherwood NE, Lehman EB, Kephart DK. Long-term weight loss maintenance in the United States. Int J Obes. 2010; 34: 1644-1654.

3. Westerterp KR. Physical activity, food intake, and body weight regulation: Insights from doubly labeled water studies. Nutr Rev. 2010; 68: 148-154.

4. Hsieh $\mathrm{CH}$. The effects of auricular acupressure on weight loss and serum lipid levels in overweight adolescents. Am J Chin Med. 2010; 38: 675-682.

5. Hsieh CH, Su TJ, Fang YW, Chou PH. Effects of auricular acupressure on weight reduction and abdominal obesity in Asian young adults: A randomized controlled trial. Am J Chin Med. 2011; 39: 433-440.

6. Hsu CH, Wang CJ, Hwang KC, Lee TY, Chou P, Chang HH. The effect of auricular acupuncture in obese women: A randomized controlled trial. J Womens Health. 2009; 18: 813-818.

7. Yeh $\mathrm{CH}$, Yeh SC. Effects of ear points' pressing on parameters related to obesity in non-obese healthy and obese volunteers. J Altern Complement Med. 2008; 14: 309-314. 
8. Cayir $Y$, Set $T$, Kosan Z. The effects of auricular and body acupuncture in Turkish obese female patients: A randomized controlled trial indicated both methods lost body weight but auricular acupuncture was better than body acupuncture. Acupunct Electrother Res. 2017; 42: 1-10.

9. Lillingston F, Fields $P$, Waechter R. Auricular acupuncture associated with reduced waist circumference in overweight women-A randomized controlled trial. Evid Based Complement Alternat Med. 2019; 2019: 6471560.

10. Mantzoros CS. Leptin and the hypothalamus: Neuroendocrine regulation of food intake. Mol Psychiatry. 1999; 4: 8-12.

11. Asamoto S, Takeshige C. Activation of the satiety center by auricular acupuncture point stimulation. Brain Res Bull. 1992; 29: 157-164.

12. Ruan Z, Xiang Y, Li J, Zhou X, Huang Z, Dong C. Auricular acupuncture for obesity: A systematic review and meta-analysis. Int J Clin Exp Med. 2016; 9: 1772-1779.

13. Nogier R. Dr. Nogier's Auriculotherapy Handbook. Houston: CBR-Publishers; 2012.

14. Sakashita T. Stress relief through auricular acupuncture. JPN J Rodoraku Med. 1992; 37: 234236.

15. Wang L, Zhao B, Zhou L. Status and strategies analysis on international standardization of auricular acupuncture points. J Tradit Chin Med. 2013; 33: 408-412.

16. Mukaino Y, Arakawa K. Change of taste by ear acupuncture in simple obese patients. J JPN Soc Acupunct Moxibustion. 1985; 34: 211-216.

17. Mukaino Y. Acupuncture therapy for obesity using ear needle treatment: analysis of effectiveness and mechanism. J Jpn Soc Acupunct Moxibustion. 1981; 31: 67-74.

18. Kageyama T, Samesima Y, Kojima T, Sawatsugawa S, Shiraishi T. Effects of auricular acupuncture stimulation on body weight in the healthy volunteer subjects. J JPN Soc Acupunct Moxibustion. 1994; 44: 339-346.

19. lino Y. Pain in Otorhinolaryngology. Nippon Jibiinkoka Gakkai Kaiho. 2014; 117: 12: 1431-1437. doi: 10.3950/jibiinkoka.117.1431.

20. Jensen MD, Ryan DH, Apovian CM, Ard JD, Comuzzie AG, Donato KA, et al. 2013 AHA/ACC/TOS guideline for the management of overweight and obesity in adults: $A$ report of the american college of cardiology/american heart association task force on practice guidelines and the obesity society. J Am Coll Cardiol. 2014; 63: 2985-3023.

21. Lacey JM, Tershakovec AM, Foster GD. Acupuncture for the treatment of obesity: A review of the evidence. Int J Obes Relat Metab Disord. 2003; 27: 419-427.

22. Kojima T, Onoue K, Sakurai K, Shiraishi T. The effect of auricular acupuncture stimulation in reducing food consumption by rats with diet-induced obesity as a model of simple obesity is dependent on improved energy metabolism. J JPN Soc Study Obesity. 2003; 9: 5137.

23. Sun $Q, X u Y$. Simple obesity and obesity hyperlipemia treated with otoacupoint pellet pressure and body acupuncture. J Tradit Chin Med. 1993; 13: 22-26. 


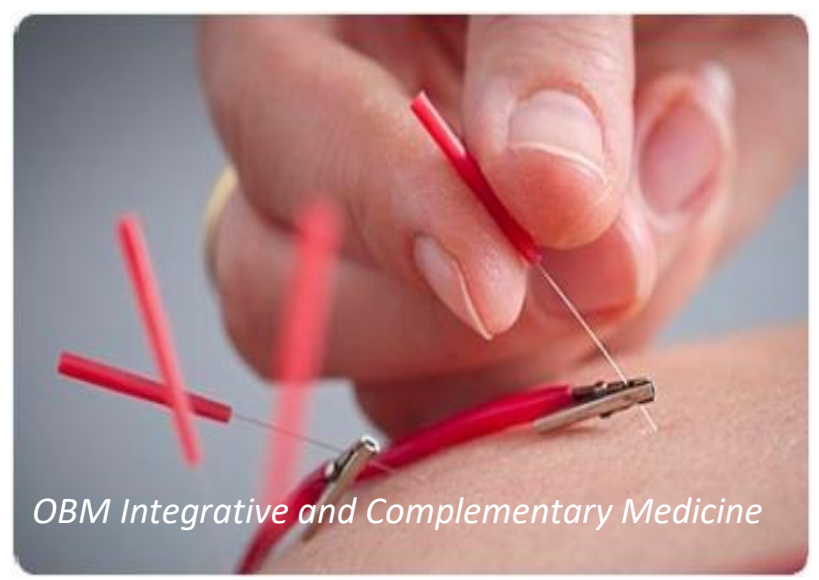

Enjoy OBM Integrative and Complementary Medicine by:

1. Submitting a manuscript

2. Joining in volunteer reviewer bank

3. Joining Editorial Board

4. Guest editing a special issue

For more details, please visit:

http://www.lidsen.com/journals/icm 


$$
\begin{gathered}
\text { LIDSEN Publishing Inc. } \\
2000 \text { Auburn Drive, One Chagrin } \\
\text { Highlands, Suite 200, Beachwood, OH } 44122 \\
\text { USA } \\
\text { Tel.: +1-216-370-7293 } \\
\text { Fax: }+1-216-378-7505 \\
\text { http://www.lidsen.com/ }
\end{gathered}
$$

OBM Integrative and Complementary Medicine Editorial Office,

E-mail: icm@lidsen.com

http://www.lidsen.com/journals/icm 
LIDSEN Publishing Inc.

2000 Auburn Drive, One Chagrin

Highlands, Suite 200, Beachwood

$\mathrm{OH} 44122$

USA

Tel.: +1-216-370-7293

Fax: +1-216-378-7505

http://www.lidsen.com/ 